Supporting Information

\title{
Sulfone as a Transient Activating Group in Palladium- Catalyzed Asymmetric [4 + 3] Cycloaddition of Trimethylenemethane Enabling the Enantioselective Synthesis of Fused Azepines
}

Yang-Zi Liu, ${ }^{*}, \#$ Zhongao Wang,,${ }^{\ddagger}$ Zesheng Huang ${ }^{\dagger}$, Wu-Lin Yang ${ }^{\dagger}$, and Wei-Ping Deng $^{*}$,

${ }^{\dagger}$ Shanghai Key Laboratory of New Drug Design \& School of Pharmacy, East China University of Science and Technology, 130 Meilong Road, Shanghai 200237, China.

" State Key Laboratory of Organometallic Chemistry, Shanghai Institute of Organic Chemistry, University of Chinese Academy of Sciences, Chinese Academy of Sciences, 345 Lingling Lu, Shanghai 200032, China

liuyangzi@sioc.ac.cn

weiping_deng@ecust.edu.cn 


\section{Table of contents}

1. General materials and methods .................................................................. 3

2. Screening of catalysts and solvents ................................................. 3

3. The stereochemistry analysis.....................................................5

4. Experimental procedure ..................................................................6 6

5. The absolute configuration determination of $(4 S, 5 S)-3 \mathrm{bq} \ldots \ldots . . . . . . . . . .16$

6. ${ }^{1} \mathrm{H}$ and ${ }^{13} \mathrm{C}$ NMR spectra .................................................................. 18

7. Chiral HPLC Chromatograms.................................................... 42 


\section{General materials and methods}

${ }^{1} \mathrm{H}$ NMR spectrum were recorded on Bruker DPX $400 \mathrm{MHz}$ spectrometer or Bruker Ascend 600 $\mathrm{MHz}$ spectrometer in $\mathrm{CDCl}_{3}$ and Acetone- $d_{6}$. Chemical shifts were reported in ppm with the internal TMS signal at $0.00 \mathrm{ppm}$ as a standard. The spectrum is interpreted as: $\mathrm{s}=\operatorname{singlet}, \mathrm{d}=$ doublet, $\mathrm{t}=$ triplet, $\mathrm{m}=$ multiplet, brs = broad singlet, $\mathrm{dd}=$ doublet of doublets, $\mathrm{dt}=$ doublet of triplets, coupling constant(s) $J$ are reported in $\mathrm{Hz}$ and relative integrations are reported. ${ }^{13} \mathrm{C}$ NMR $(100 \mathrm{MHz})$ spectrum were recorded on Bruker DPX $400 \mathrm{MHz}$ spectrometer in $\mathrm{CDCl}_{3}$ and Acetone- $d_{6} .{ }^{13} \mathrm{C}$ NMR spectra were referenced to the solvent resonance $\left(\mathrm{CDCl}_{3}\right.$ at $77.06 \pm 0.03 \mathrm{ppm}$ and Acetone- $d_{6}$ at $29.82 \pm$ $0.01 \mathrm{ppm})$. Optical rotations were measured on an AUTOPOL V. Diastereomeric ratios were determined from crude ${ }^{1} \mathrm{H}$ NMR spectroscopy interpretation or by analysis of HPLC traces. Enantiomer ratios were determined by analysis of HPLC traces, obtained by using Chiralpak IA or IF or AD-H or IB columns with $n$-hexane, $i$-propanol and ethanol as solvents. (Chiralpak IA, IF, AD-H and IB columns were purchased from Daicel Chemical Industries, LTD.) Melting points were obtained in open capillary tubes using SGW X-4 micro melting point apparatus which were uncorrected. Mass spectrum were recorded on TOF mass spectrometer. Absolute configuration of product was measured in D8 Venture X-ray single crystal diffractometer. Commercially available materials purchased from Adamas-beta, TCI or Energy Chemical and were used as received. Tetrahydrofuran was distilled over sodium, dichloromethane was distilled over calcium hydride.

\section{Screening of catalysts and solvents}
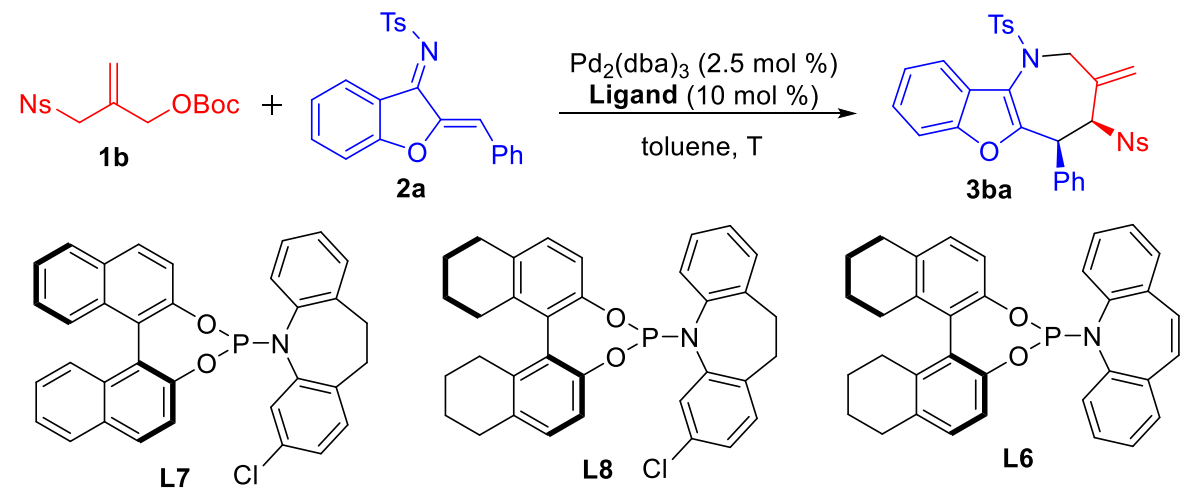

\begin{tabular}{ccccccc}
\hline entry $^{[\mathbf{a}]}$ & ligand & solvent & $\mathbf{T}\left({ }^{\circ} \mathbf{C}\right)$ & $\mathbf{y i e l d}(\%)^{[\mathbf{b}]}$ & $\mathbf{d r}^{[\mathrm{c}]}$ & $\mathbf{e e}^{(\%))^{[\mathrm{d}]}}$ \\
\hline 1 & $\mathbf{L 7}$ & Toluene & 40 & 73 & $1: 2$ & $88 / 91$ \\
2 & $\mathbf{L 8}$ & Toluene & 40 & 80 & $1: 1$ & $74 / 31$ \\
3 & L6 & DCM:THF=2:1 & -20 & 70 & $16: 1$ & 98 \\
4 & L6 & DCM:THF=1:2 & -20 & 83 & $18: 1$ & 99
\end{tabular}

[a] The reaction was carried out by using $\mathbf{2 a}(0.1 \mathrm{mmol}), \mathbf{1 b}(0.12 \mathrm{mmol})$ in $1.0 \mathrm{~mL}$ of solvent. [b] Isolated yield. [c] The diastereomeric ratio (dr) was determined by ${ }^{1} \mathrm{H}$ NMR., [d] The ee value was 
determined by HPLC analysis on a chiral stationary phase. 


\section{The stereochemistry analysis}

We propose two possible configurations separately (Config. A and B) for key intermediate to explain the opposite diastereoselectivity when using $\mathbf{L 5}$ and L6. For the Carreira Ligand L5 derived Pd-TMM species, as depicted in Fig. S1a, the sulfonyl group of $\mathbf{1 b}$ presumably forms $\pi$ - $\pi$ stacking effect simultaneously with iminostilbene and BINOL motif of the ligand L5, resulting in the competition of two favorable configurations, and the configuration $\mathrm{A}$ is more favorable (Fig. S1a, Config. A and B). This may be one of the reasons for the low dr value of the product when using ligand L5. However, for the $\mathrm{H}_{8}$-BINOL-Carreira Ligand L6 derived Pd-TMM species (Fig. S1b), compared with $\mathbf{L 5}$, the sulfonyl group of $\mathbf{1 b}$ only forms $\pi$ - $\pi$ stacking effect with iminostilbene group of the ligand L6, and has a steric hindrance effect with $\mathrm{H}_{8}$-BINOL component of the ligand L6 instead of $\pi-\pi$ stacking effect, which makes the configuration B more favorable (Fig. S1b, Config. B). This is the key factor that causes the opposite diastereoselectivity when using $\mathbf{L 5}$ and L6.

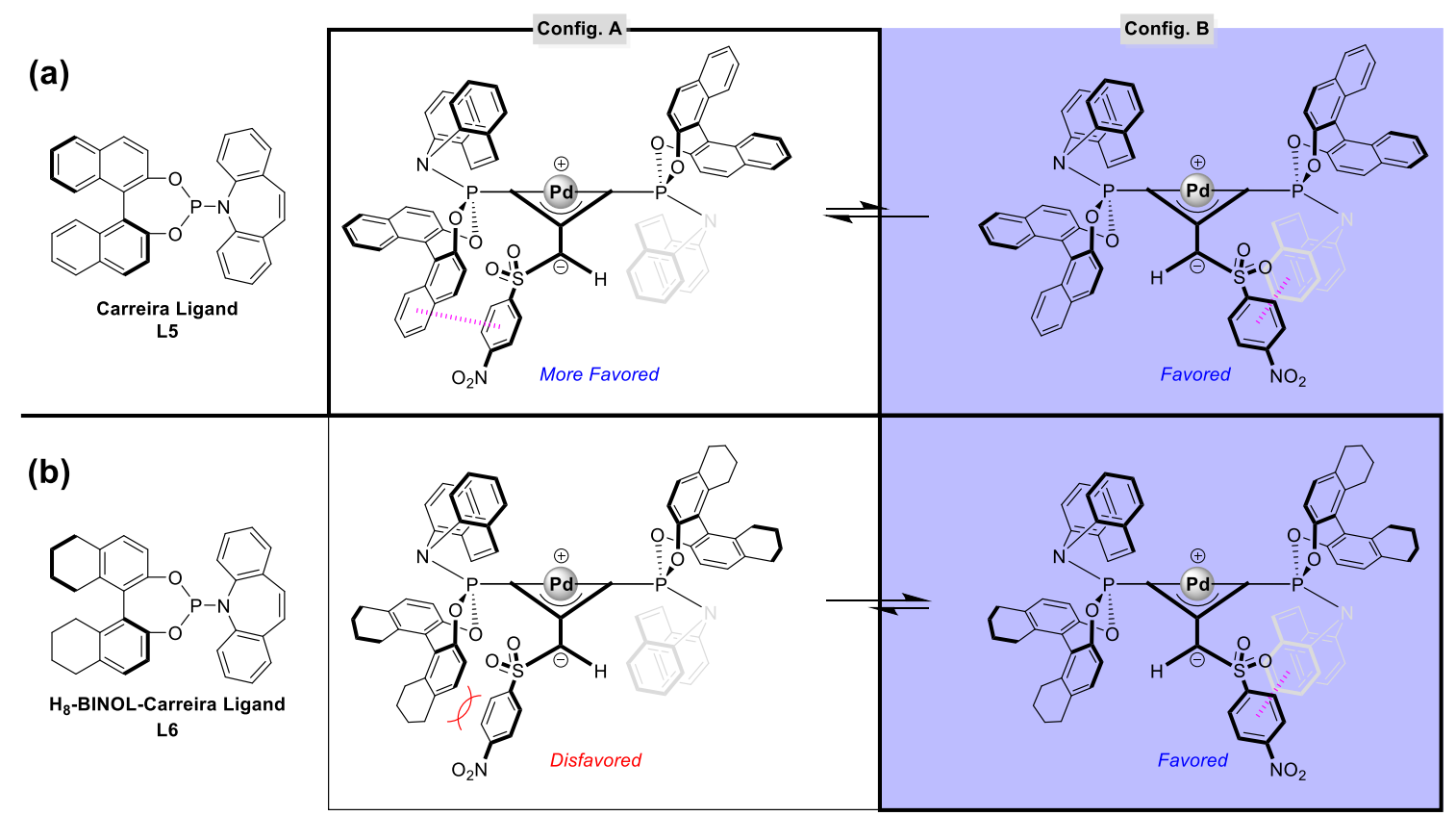

Fig S1. The stereochemistry analysis. 


\section{Experimental procedure}

All of the benzofuran-derived azadienes $\mathbf{2}$ are known compounds, which are prepared according to the literature procedure ${ }^{[1]}$.

References:

[1] H. Ni.; X. Tang.; W. Zheng.; W. Yao.; N. Ullah.; Y. Lu. Angew. Chem. Int. Ed. 2017, 56,14222 .

\section{Procedure for the synthesis of sulfonyl-TMM donor $1 \mathrm{~b}$}

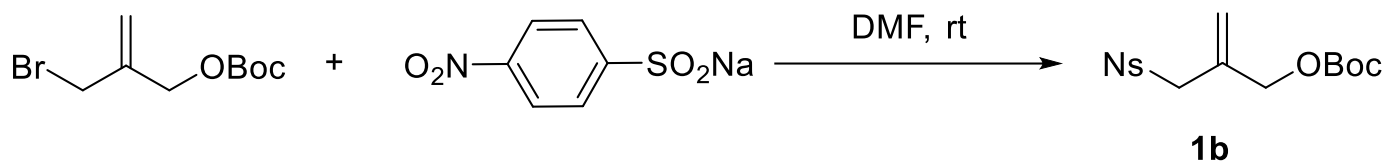

Under a nitrogen atmosphere, 2-(bromomethyl)allyl tert-butyl carbonate $(0.5 \mathrm{~g}, 2 \mathrm{mmol})$ and sodium 4-nitrobenzenesulfinate $(0.5 \mathrm{~g}, 2.4 \mathrm{mmol})$ were dissolved in DMF $(8.0 \mathrm{~mL})$, and stirred at room temperature until 2-(bromomethyl)allyl tert-butyl carbonate was consumed. Then add $100 \mathrm{~mL}$ ethyl acetate to the reaction, the organic layer was washed with water ( $2 \mathrm{~mL} \times 4$ times), after that the organic layer was washed with brine, dried over $\mathrm{Na}_{2} \mathrm{SO}_{4}$, the mixture was concentrated and purified by column chromatography (petroleum ether/ethyl acetate $=6: 1$ ) on silica gel to afford $\mathbf{1 b}$

Ns _OBoc $\begin{aligned} & \text { compound 1b, white solid, } 0.5 \mathrm{~g}, 70 \% \text { yield, m.p.: 120-122 }{ }^{\circ} \mathrm{C} ;{ }^{1} \mathbf{H} \text { NMR }(400 \\ & \mathrm{MHz}, \text { Chloroform- } d) \delta 8.42(\mathrm{~s}, 1 \mathrm{H}), 8.40(\mathrm{~s}, 1 \mathrm{H}), 8.12(\mathrm{~s}, 1 \mathrm{H}), 8.10(\mathrm{~s}, 1 \mathrm{H}) \text {, }\end{aligned}$ $5.44(\mathrm{~s}, 1 \mathrm{H}), 5.05(\mathrm{~s}, 1 \mathrm{H}), 4.64(\mathrm{~s}, 2 \mathrm{H}), 3.94$ (s, 2H), 1.48 (s, 9H); ${ }^{13} \mathrm{C}$ NMR (100 MHz, Chloroformd) $\delta 152.9,151.0,143.4,131.5,130.2(2 \mathrm{C}), 124.3$ (2C), 123.9, 82.9, 67.7, 59.8, 27.7 (3C); HRMS (ESI-TOF) m/z: [M+Na] $]^{+}$calcd for $\left[\mathrm{C}_{15} \mathrm{H}_{19} \mathrm{NO}_{7} \mathrm{SNa}\right]^{+}$: 380.0780; found: 380.0774 .

General procedure for the asymmetric $[4+3]$ cyclization of sulfonyl-TMM donor 1b with benzofuran-derived azadienes 2

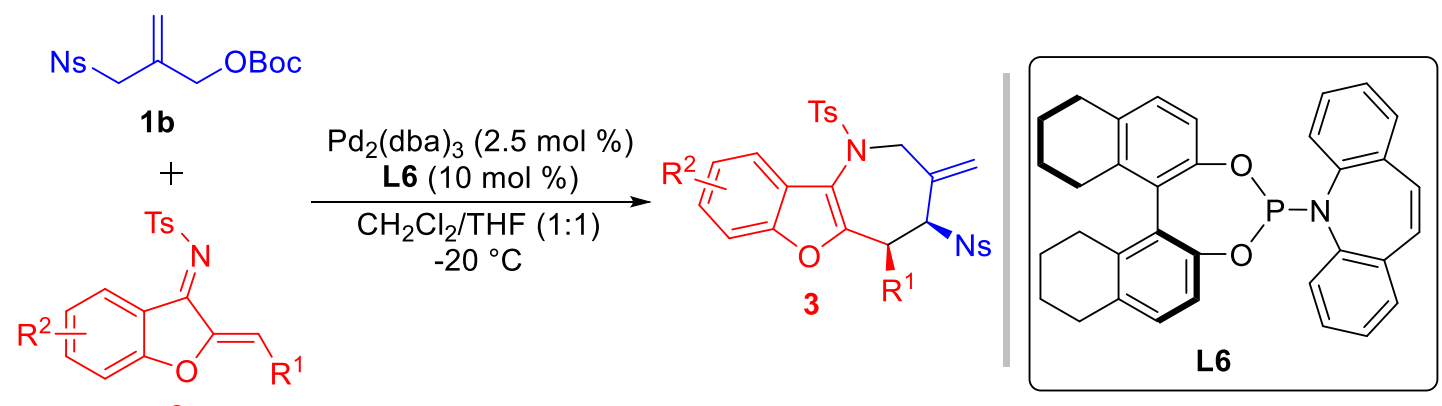

Under a nitrogen atmosphere, $\mathrm{Pd}_{2}(\mathrm{dba})_{3}(2.3 \mathrm{mg}, 0.0025 \mathrm{mmol})$ and ligand $\mathbf{L 6}(5.2 \mathrm{mg}, 0.01 \mathrm{mmol})$ were dissolved in DCM/THF $=1: 1(\mathrm{v} / \mathrm{v}=1: 1,1.0 \mathrm{~mL})$, and stirred at room temperature for 
approximately $30 \mathrm{~min}$. Then the mixture was cooled to $-20^{\circ} \mathrm{C}$ and sulfonyl-TMM donor $\mathbf{1 b}$ (53.6 $\mathrm{mg}, 0.15 \mathrm{mmol})$ and benzofuran-derived azadienes $2(0.1 \mathrm{mmol})$ were added sequentially. Once starting material was consumed (monitored by TLC), the solvent was evaporated. The mixture was concentrated and purified by column chromatography to give the corresponding cycloaddition products.

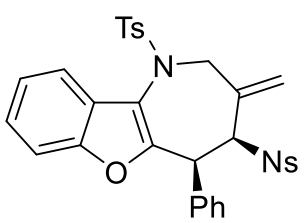

compound 3ba, white solid, $49.2 \mathrm{mg}$, yield $80 \%$, m.p.: $142-144{ }^{\circ} \mathrm{C} ;[\alpha]_{\mathrm{D}}{ }^{20}=$ $+15.8\left(\mathrm{CH}_{2} \mathrm{Cl}_{2}, c=1.00\right) ;{ }^{1} \mathrm{H}$ NMR $(400 \mathrm{MHz}$, Chloroform- $d) \delta 8.33(\mathrm{~d}, J=$ $8.5 \mathrm{~Hz}, 2 \mathrm{H}), 7.92(\mathrm{~d}, J=8.4 \mathrm{~Hz}, 2 \mathrm{H}), 7.63(\mathrm{~d}, J=8.0 \mathrm{~Hz}, 2 \mathrm{H}), 7.39-7.27(\mathrm{~m}$, $6 \mathrm{H}), 7.25-7.20(\mathrm{~m}, 3 \mathrm{H}), 7.18-7.11(\mathrm{~m}, 2 \mathrm{H}), 5.53-5.31(\mathrm{~m}, 3 \mathrm{H}), 4.93(\mathrm{~s}$, 1H), $4.43(\mathrm{~d}, J=16.3 \mathrm{~Hz}, 1 \mathrm{H}), 4.15(\mathrm{~d}, J=16.3 \mathrm{~Hz}, 1 \mathrm{H}), 2.50(\mathrm{~s}, 3 \mathrm{H}) ;{ }^{13} \mathrm{C}$ NMR $(100 \mathrm{MHz}$, Chloroform-d) $\delta 153.3,151.1,150.7,144.8,144.6,136.4,134.4,133.0,130.8$ (2C), 130.3 (2C), 130.1 (2C), 128.5, 127.8 (2C), 127.5 (2C), 125.8, 125.0, 124.0 (2C), 123.3, 122.2, 119.8, 119.3, 111.7, 64.1, 57.5, 44.2, 21.7; HRMS (ESI-TOF) m/z: $[\mathrm{M}-\mathrm{H}]^{+}$calcd for $\left[\mathrm{C}_{32} \mathrm{H}_{25} \mathrm{~N}_{2} \mathrm{O}_{7} \mathrm{~S}_{2}\right]^{+}$: 613.1098; found: 613.1102. HPLC (Chiralpak IA, $n$-hexane $/ i$-propanol $=7 / 3,1.0 \mathrm{~mL} / \mathrm{min}, 220 \mathrm{~nm}$ ) $\mathrm{t}_{\mathrm{R}}=12.98 \mathrm{~min}$, $21.23 \mathrm{~min}$.

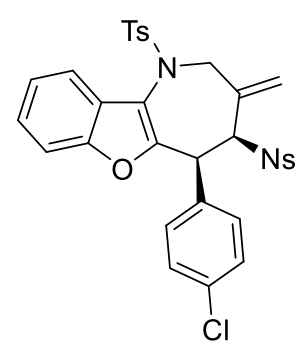

compound 3bb, white solid, $60.4 \mathrm{mg}$, yield $93 \%$, m.p.: $154-156^{\circ} \mathrm{C} ;[\alpha]_{\mathrm{D}}{ }^{20}$ $=+5.8\left(\mathrm{CH}_{2} \mathrm{Cl}_{2}, c=1.00\right) ;{ }^{1} \mathbf{H}$ NMR $(400 \mathrm{MHz}$, Chloroform- $d) \delta 8.38-8.31$ $(\mathrm{m}, 2 \mathrm{H}), 7.95(\mathrm{~d}, J=8.6 \mathrm{~Hz}, 2 \mathrm{H}), 7.62(\mathrm{~d}, J=8.1 \mathrm{~Hz}, 2 \mathrm{H}), 7.35(\mathrm{~d}, J=8.0 \mathrm{~Hz}$, 2H), $7.33-7.26(\mathrm{~m}, 3 \mathrm{H}), 7.26-7.23(\mathrm{~m}, 1 \mathrm{H}), 7.18-7.12(\mathrm{~m}, 4 \mathrm{H}), 5.60-$ $5.30(\mathrm{~m}, 3 \mathrm{H}), 4.98$ (s, 1H), $4.40(\mathrm{~d}, J=16.4 \mathrm{~Hz}, 1 \mathrm{H}), 4.13(\mathrm{~d}, J=16.4 \mathrm{~Hz}$, 1H), 2.51 (s, 3H); ${ }^{13}$ C NMR (100 MHz, Chloroform- $d$ ) $\delta$ 153.3, 150.8, 150.6, 144.9, 144.3, 136.3 134.5, 133.0, 132.8, 132.1 (2C), 130.4 (2C), 130.2 (2C), 128.0 (2C), 127.6 (2C), 125.7, 125.3, 124.1 (2C), 123.4, 122.1, 119.8, 119.5, 111.7, 63.3, 57.7, 43.6, 21.7; HRMS (ESI-TOF) m/z: $[\mathrm{M}-\mathrm{H}]^{+}$calcd for $\left[\mathrm{C}_{32} \mathrm{H}_{24} \mathrm{ClN}_{2} \mathrm{O}_{7} \mathrm{~S}_{2}\right]^{+}$: 647.0708; found: 647.0714. HPLC (Chiralpak AD, $n$ hexane $/ i$-propanol $=6 / 4,1.0 \mathrm{~mL} / \mathrm{min}, 220 \mathrm{~nm}) \mathrm{t}_{\mathrm{R}}=11.08 \mathrm{~min}, 31.45 \mathrm{~min}$.

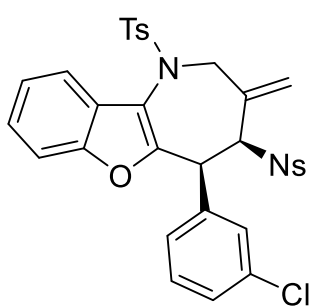

compound 3bc, white solid , $60.4 \mathrm{mg}$, yield 93\%, m.p.: $150-152{ }^{\circ} \mathrm{C} ;[\alpha]_{\mathrm{D}}{ }^{20}=$ $+25.7\left(\mathrm{CH}_{2} \mathrm{Cl}_{2}, c=1.00\right) ;{ }^{1} \mathrm{H}$ NMR $(400 \mathrm{MHz}$, Chloroform- $d$ ) $\delta 8.40-8.30$ $(\mathrm{m}, 2 \mathrm{H}), 7.94(\mathrm{~d}, J=8.7 \mathrm{~Hz}, 2 \mathrm{H}), 7.62(\mathrm{~d}, J=8.1 \mathrm{~Hz}, 2 \mathrm{H}), 7.37-7.22(\mathrm{~m}$, $7 \mathrm{H}), 7.18-7.15(\mathrm{~m}, 2 \mathrm{H}), 7.11-7.09(\mathrm{~m}, 1 \mathrm{H}), 5.44-5.35(\mathrm{~m}, 3 \mathrm{H}), 4.94(\mathrm{~s}$, $1 \mathrm{H}), 4.45(\mathrm{~d}, J=16.4 \mathrm{~Hz}, 1 \mathrm{H}), 4.15(\mathrm{~d}, J=16.4 \mathrm{~Hz}, 1 \mathrm{H}), 2.50(\mathrm{~s}, 3 \mathrm{H}) ;{ }^{13} \mathrm{C}$ NMR (100 MHz, Chloroform- $d$ ) $\delta$ 153.4, 150.9, 150.3, 144.9, 144.4, 136.4, 136.3, 133.6, 133.2, 130.5, 130.3 (2C), 130.1 (2C), 129.3, 129.1, 128.7, 127.6 (2C), 125.7, 125.3, 124.1 (2C), 123.4, 122.4, 119.9, 119.6, 111.7, 63.9, 57.5, 43.8, 21.7; HRMS (ESI-TOF) $\mathrm{m} / \mathrm{z}:[\mathrm{M}-\mathrm{H}]^{+}$calcd for $\left[\mathrm{C}_{32} \mathrm{H}_{24} \mathrm{ClN}_{2} \mathrm{O}_{7} \mathrm{~S}_{2}\right]^{+}:$647.0708; found: 647.0714. HPLC (Chiralpak IA, $n$-hexane/i-propanol = 7/3, 1.0 $\mathrm{mL} / \mathrm{min}, 220 \mathrm{~nm}) \mathrm{t}_{\mathrm{R}}=13.66 \mathrm{~min}, 18.08 \mathrm{~min}$. 


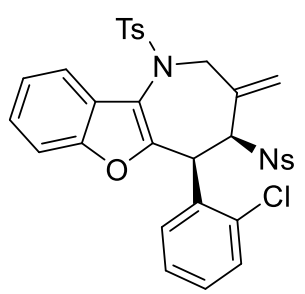

compound 3bd, white solid, $54.4 \mathrm{mg}$, yield 84\%; m.p.: $157-158{ }^{\circ} \mathrm{C} ;{ }^{1} \mathbf{H}$ NMR (400 MHz, Chloroform- $d$ ) $\delta 8.22(\mathrm{~d}, J=8.7 \mathrm{~Hz}, 2 \mathrm{H}$, major), $8.16(\mathrm{~d}, J$ $=8.4 \mathrm{~Hz}, 1 \mathrm{H}$, minor), 8.01 (s, $1 \mathrm{H}$, minor), $7.92(\mathrm{~d}, J=8.0 \mathrm{~Hz}, 1 \mathrm{H}$, minor), $7.85(\mathrm{~d}, J=8.4 \mathrm{~Hz}, 1 \mathrm{H}$, minor), $7.75(\mathrm{~d}, J=8.4 \mathrm{~Hz}, 2 \mathrm{H}$, major), $7.58-7.53$ $(\mathrm{m}, 3 \mathrm{H}$, major + minor), $7.43(\mathrm{~m}, 2 \mathrm{H}$, major $), 7.40-7.34(\mathrm{~m}, 1 \mathrm{H}$, minor $)$, $7.32-7.15(\mathrm{~m}, 9 \mathrm{H}$, major + minor), $7.07(\mathrm{~m}, 0.5 \mathrm{H}$, minor $), 6.91(\mathrm{~s}, 0.5 \mathrm{H}$, minor), $5.45(\mathrm{~m}, 2 \mathrm{H}$, major + minor), $5.34(\mathrm{~m}, 0.5 \mathrm{H}$, minor), 5.24 (s, 1H, major), 5.14 (s, 1H, major), 4.87 (s, $0.5 \mathrm{H}$, major), 4.76 (d, $J=15.6 \mathrm{~Hz}, 1 \mathrm{H}$, major), 4.72 (s, 0.5H, minor), 4.46 (s, 1H, major), 4.37 (s, $0.5 \mathrm{H}$, minor), 4.27 (d, $J=15.8 \mathrm{~Hz}, 1 \mathrm{H}$, major), 2.52 (s, 1.5H, minor), 2.41 (s, 3H); ${ }^{13} \mathbf{C}$ NMR (100 MHz, Chloroform- $d$ ) $\delta 153.3,150.5,144.8,144.6,136.4,135.9,133.6,132.0,130.2,129.9$ (2C), 129.6, 129.5(4) , 129.4(8) (2C), 127.7, 127.5 (2C), 126.0, 125.7, 125.0, 124.0 (2C), 123.6, 120.5, 120.2, 119.9, 111.5, 68.1, 53.8, 40.5, 21.6; HRMS (ESI-TOF) $\mathrm{m} / \mathrm{z}:[\mathrm{M}-\mathrm{H}]^{+}$calcd for $\left[\mathrm{C}_{32} \mathrm{H}_{24} \mathrm{ClN}_{2} \mathrm{O}_{7} \mathrm{~S}_{2}\right]^{+}:$647.0708; found: 647.0716. HPLC (Chiralpak AD, $n$-hexane/i-propanol $=6 / 4$, $1.0 \mathrm{~mL} / \mathrm{min}, 220 \mathrm{~nm}$ ) $\mathrm{t}_{\mathrm{R}}=16.42 \mathrm{~min}$ (cis-minor), $17.24 \mathrm{~min}$ (cis-major), $22.53 \mathrm{~min}$ (trans-minor), 56.23 min (trans-major).

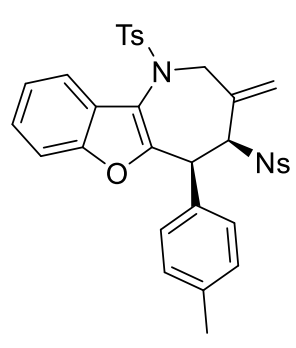

compound 3be, white solid, $56.0 \mathrm{mg}$, yield $89 \%$, m.p.: $136-137{ }^{\circ} \mathrm{C} ;[\alpha]_{\mathrm{D}}{ }^{20}=$ $+25.2\left(\mathrm{CH}_{2} \mathrm{Cl}_{2}, c=1.00\right) ;{ }^{1} \mathbf{H}$ NMR $(400 \mathrm{MHz}$, Chloroform- $d) \delta 8.33(\mathrm{~d}, J=$ $8.4 \mathrm{~Hz}, 2 \mathrm{H}), 7.92(\mathrm{~d}, J=8.4 \mathrm{~Hz}, 2 \mathrm{H}), 7.62(\mathrm{~d}, J=8.0 \mathrm{~Hz}, 2 \mathrm{H}), 7.34(\mathrm{~d}, J=$ $8.0 \mathrm{~Hz}, 2 \mathrm{H}), 7.32-7.28(\mathrm{~m}, 1 \mathrm{H}), 7.26-7.08(\mathrm{~m}, 7 \mathrm{H}), 5.51-5.28(\mathrm{~m}, 3 \mathrm{H})$, $4.96-4.85(\mathrm{~m}, 1 \mathrm{H}), 4.42(\mathrm{~d}, J=16.4 \mathrm{~Hz}, 1 \mathrm{H}), 4.15(\mathrm{~d}, J=16.3 \mathrm{~Hz}, 1 \mathrm{H})$, $2.50(\mathrm{~s}, 3 \mathrm{H}), 2.35$ (s, 3H); ${ }^{13} \mathrm{C}$ NMR (100 MHz, Chloroform- $\left.d\right) \delta$ 153.3, 151.3, 150.7, 144.8, 144.6, 138.3, 136.4, 133.1, 131.4, 130.6 (2C), 130.3 (2C), 130.1 (2C), 128.5 (2C), 127.5 (2C), 125.8, 125.0, 124.0 (2C), 123.2, 122.2, 119.8, 119.2, 111.7, 64.2, 57.5, 43.9, 21.7, 21.3; HRMS (ESI-TOF) m/z: $[\mathrm{M}-\mathrm{H}]^{+}$calcd for $\left[\mathrm{C}_{33} \mathrm{H}_{27} \mathrm{~N}_{2} \mathrm{O}_{7} \mathrm{~S}_{2}\right]^{+}$: 627.1254; found: 627.1262. HPLC $($ Chiralpak IB, $n$-hexane/ethanol $=8 / 2,1.0 \mathrm{~mL} / \mathrm{min}, 220 \mathrm{~nm}) \mathrm{t}_{\mathrm{R}}=13.88 \mathrm{~min}, 18.07 \mathrm{~min}$.

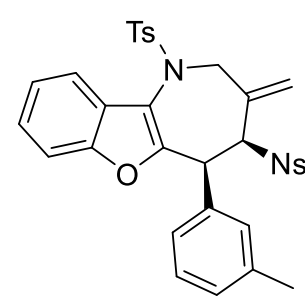

compound 3bf, white solid, $57.2 \mathrm{mg}$, yield $91 \%$, m.p.: $136-138^{\circ} \mathrm{C} ;[\alpha]_{\mathrm{D}}{ }^{20}=$ $+18.7\left(\mathrm{CH}_{2} \mathrm{Cl}_{2}, c=1.00\right) ;{ }^{1} \mathbf{H}$ NMR $(400 \mathrm{MHz}$, Chloroform- $d) \delta 8.36-$ $8.27(\mathrm{~m}, 2 \mathrm{H}), 7.92(\mathrm{~d}, J=8.6 \mathrm{~Hz}, 2 \mathrm{H}), 7.63(\mathrm{~d}, J=8.1 \mathrm{~Hz}, 2 \mathrm{H}), 7.37-7.29$ (m, 3H), $7.26-7.12(\mathrm{~m}, 5 \mathrm{H}), 7.05(\mathrm{~s}, 1 \mathrm{H}), 7.01-6.98(\mathrm{~m}, 1 \mathrm{H}), 5.41-5.33$ (m, 3H), $4.90(\mathrm{~s}, 1 \mathrm{H}), 4.44(\mathrm{~d}, J=16.4 \mathrm{~Hz}, 1 \mathrm{H}), 4.16(\mathrm{~d}, J=16.3 \mathrm{~Hz}, 1 \mathrm{H})$, 2.50 (s, 3H), 2.33 (s, 3H); ${ }^{13}$ C NMR (100 MHz, Chloroform- $d$ ) $\delta$ 153.4, $151.3,150.8,144.8,144.7,137.4,136.4,134.3,133.3,131.5,130.3$ (2C), 130.1 (2C), 129.3, 127.8, 127.6(2), 127.5(8) (2C), 125.8, 125.0, 124.0 (2C), 123.3, 122.2, 119.9, 119.2, 111.7, 64.3, 57.4, 44.2, 21.7, 21.5; HRMS (ESI-TOF) m/z: [M-H] $]^{+}$calcd for $\left[\mathrm{C}_{33} \mathrm{H}_{27} \mathrm{~N}_{2} \mathrm{O}_{7} \mathrm{~S}_{2}\right]^{+}:$: 627.1254; found: 627.1262. HPLC (Chiralpak IA, $n$-hexane $/ i$-propanol $=7 / 3,1.0 \mathrm{~mL} / \mathrm{min}, 220 \mathrm{~nm}$ ) $\mathrm{t}_{\mathrm{R}}=$ 


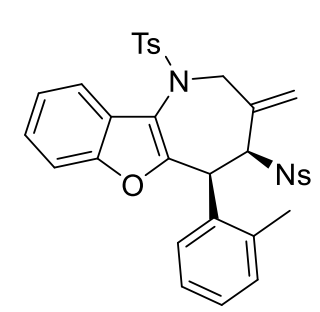

compound 3bg, white solid, $53.4 \mathrm{mg}$, yield $85 \%$, m.p.: $133-135^{\circ} \mathrm{C} ;[\alpha]_{\mathrm{D}}{ }^{20}=$ $+27.5\left(\mathrm{CH}_{2} \mathrm{Cl}_{2}, c=1.00\right) ;{ }^{1} \mathbf{H}$ NMR $(400 \mathrm{MHz}$, Chloroform- $d$ ) $\delta 8.34-8.31$ $(\mathrm{m}, 2 \mathrm{H}), 7.92(\mathrm{~d}, J=8.5 \mathrm{~Hz}, 2 \mathrm{H}), 7.62(\mathrm{~d}, J=8.1 \mathrm{~Hz}, 2 \mathrm{H}), 7.38-7.28(\mathrm{~m}$, $3 \mathrm{H}), 7.27-7.12(\mathrm{~m}, 5 \mathrm{H}), 7.05(\mathrm{~s}, 1 \mathrm{H}), 7.02-6.98(\mathrm{~m}, 1 \mathrm{H}), 5.41-5.23(\mathrm{~m}$, 3H), 4.89 (s, 1H), 4.44 (d, $J=16.4 \mathrm{~Hz}, 1 \mathrm{H}), 4.16$ (d, $J=16.3 \mathrm{~Hz}, 1 \mathrm{H}), 2.50$ (s, 3H), 2.33 (s, 3H); ${ }^{13}$ C NMR (100 MHz, Chloroform- $d$ ) $\delta$ 153.4, 151.3, 150.7, 144.8, 144.7, 137.4, 136.4, 134.3, 133.2, 131.5, 130.3 (2C), 130.1 (2C), 129.3, 127.8, 127.6(4), 127.5(8) (2C), 125.8, 125.0, 124.0 (2C), 123.3, 122.2, 119.9, 119.2, 111.7, 64.4, 57.4, 44.2, 21.7, 21.5; HRMS (ESI-TOF) m/z: $[\mathrm{M}-\mathrm{H}]^{+}$calcd for $\left[\mathrm{C}_{33} \mathrm{H}_{27} \mathrm{~N}_{2} \mathrm{O}_{7} \mathrm{~S}_{2}\right]^{+}$: 627.1254; found: 627.1258. HPLC (Chiralpak IA, $n$ hexane $/ i$-propanol $=7 / 3,1.0 \mathrm{~mL} / \mathrm{min}, 220 \mathrm{~nm}) \mathrm{t}_{\mathrm{R}}=10.72 \mathrm{~min}, 13.28 \mathrm{~min}$.

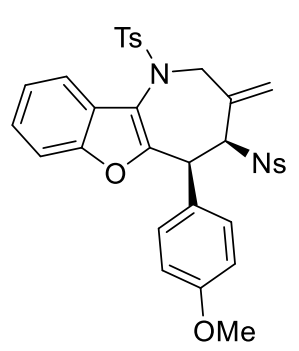

compound 3bh, white solid, $46.4 \mathrm{mg}$, yield $72 \%$, m.p.: $129-130{ }^{\circ} \mathrm{C} ;[\alpha]_{\mathrm{D}}^{20}=$ $+18.6\left(\mathrm{CH}_{2} \mathrm{Cl}_{2}, c=1.00\right) ;{ }^{1} \mathbf{H}$ NMR $(400 \mathrm{MHz}$, Chloroform- $d) \delta 8.32(\mathrm{~d}, J=$ $8.4 \mathrm{~Hz}, 2 \mathrm{H}), 7.92(\mathrm{~d}, J=8.4 \mathrm{~Hz}, 2 \mathrm{H}), 7.62(\mathrm{~d}, J=7.9 \mathrm{~Hz}, 2 \mathrm{H}), 7.37-7.27(\mathrm{~m}$, $3 \mathrm{H}), 7.25-7.10(\mathrm{~m}, 5 \mathrm{H}), 6.83(\mathrm{~d}, J=8.3 \mathrm{~Hz}, 2 \mathrm{H}), 5.47-5.32(\mathrm{~m}, 3 \mathrm{H}), 4.87$ (s, 1H), $4.41(\mathrm{~d}, J=16.3 \mathrm{~Hz}, 1 \mathrm{H}), 4.14(\mathrm{~d}, J=16.2 \mathrm{~Hz}, 1 \mathrm{H}), 3.79$ (s, 3H), 2.50 (s, 3H); ${ }^{13}$ C NMR (100 MHz, Chloroform- $d$ ) $\delta$ 159.6, 153.3, 151.5, 150.8, 144.8, 144.6, 136.4, 133.1, 131.9 (2C), 130.3 (2C), 130.1 (2C), 127.6 (2C), 126.4, 125.8, 125.0, 124.0 (2C), 123.2, 122.2, 119.8, 119.1, 113.2 (2C), 111.7, 64.1, 57.6, 55.2, 43.6, 21.7; HRMS (ESI-TOF) m/z: $[\mathrm{M}-\mathrm{H}]^{+}$calcd for $\left[\mathrm{C}_{33} \mathrm{H}_{27} \mathrm{~N}_{2} \mathrm{O}_{8} \mathrm{~S}_{2}\right]^{+}$: 643.1203; found: 643.1208. HPLC (Chiralpak IA, $n$ hexane $/ i$-propanol $=7 / 3,1.0 \mathrm{~mL} / \mathrm{min}, 220 \mathrm{~nm}) \mathrm{t}_{\mathrm{R}}=13.66 \mathrm{~min}, 18.08 \mathrm{~min}$.

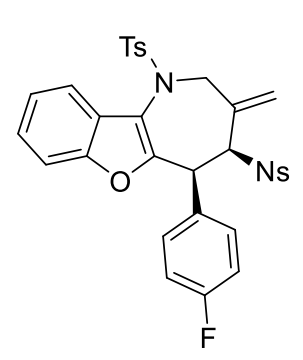

compound 3bi, white solid, $58.1 \mathrm{mg}$, yield 92\%, m.p.: $140-141{ }^{\circ} \mathrm{C} ;[\alpha]_{\mathrm{D}}{ }^{20}=$ $+11.7\left(\mathrm{CH}_{2} \mathrm{Cl}_{2}, c=1.00\right) ;{ }^{1} \mathbf{H}$ NMR $(400 \mathrm{MHz}$, Chloroform- $d$ ) $\delta 8.35(\mathrm{~d}, J=8.5$ $\mathrm{Hz}, 2 \mathrm{H}), 7.95(\mathrm{~d}, J=8.4 \mathrm{~Hz}, 2 \mathrm{H}), 7.63(\mathrm{~d}, J=7.9 \mathrm{~Hz}, 2 \mathrm{H}), 7.35$ (d, $J=8.0 \mathrm{~Hz}$, $2 \mathrm{H}), 7.32-7.27(\mathrm{~m}, 1 \mathrm{H}), 7.27-7.11(\mathrm{~m}, 5 \mathrm{H}), 7.05-6.95(\mathrm{~m}, 2 \mathrm{H}), 5.51-5.36$ $(\mathrm{m}, 3 \mathrm{H}), 4.96(\mathrm{~s}, 1 \mathrm{H}), 4.41(\mathrm{~d}, J=16.4 \mathrm{~Hz}, 1 \mathrm{H}), 4.13(\mathrm{~d}, J=16.3 \mathrm{~Hz}, 1 \mathrm{H}), 2.51$ (s, 3H); ${ }^{13}$ C NMR (100 MHz, Chloroform- $d$ ) $\delta 162.8(\mathrm{~d}, J=247.6 \mathrm{~Hz}), 153.3$, 150.9 (d, $J=5.9 \mathrm{~Hz}, 2 \mathrm{C}), 144.9,144.4,136.4,132.9,132.5$ (d, $J=8.2 \mathrm{~Hz}, 2 \mathrm{C}), 130.4$ (2C), 130.3 (d, $J=3.3 \mathrm{~Hz}), 130.2$ (2C), 127.6 (2C), 125.7, 125.2, 124.1 (2C), 123.4, 122.1, 119.8, 119.3, 114.9, 114.7, 111.7, 63.5, 57.7, 43.5, 21.7; HRMS (ESI-TOF) $\mathrm{m} / \mathrm{z}:[\mathrm{M}-\mathrm{H}]^{+}$calcd for $\left[\mathrm{C}_{32} \mathrm{H}_{24} \mathrm{FN}_{2} \mathrm{O}_{7} \mathrm{~S}_{2}\right]^{+}$: 631.1003; found: 631.1007. HPLC (Chiralpak IF, $n$-hexane $/ i$-propanol $=7 / 3,1.0 \mathrm{~mL} / \mathrm{min}, 220 \mathrm{~nm}$ ) $\mathrm{t}_{\mathrm{R}}=12.28 \mathrm{~min}, 21.11 \mathrm{~min}$. 


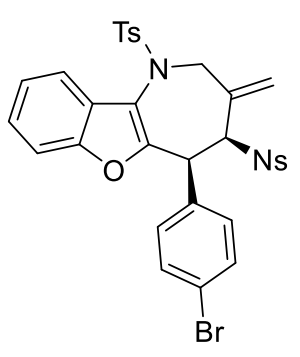

compound 3bj, white solid, $62.4 \mathrm{mg}$, yield $90 \%$, m.p.: $149-151{ }^{\circ} \mathrm{C} ;[\alpha]_{\mathrm{D}}{ }^{20}=$ $+2.2\left(\mathrm{CH}_{2} \mathrm{Cl}_{2}, c=1.00\right) ;{ }^{1} \mathbf{H}$ NMR $(400 \mathrm{MHz}$, Chloroform- $d) \delta 8.34(\mathrm{~d}, J=8.9$ $\mathrm{Hz}, 2 \mathrm{H}), 7.94(\mathrm{~d}, J=8.5 \mathrm{~Hz}, 2 \mathrm{H}), 7.62(\mathrm{~d}, J=8.1 \mathrm{~Hz}, 2 \mathrm{H}), 7.51-7.42(\mathrm{~m}, 2 \mathrm{H})$, $7.35(\mathrm{~d}, J=8.0 \mathrm{~Hz}, 2 \mathrm{H}), 7.31-7.20(\mathrm{~m}, 2 \mathrm{H}), 7.15-7.12(\mathrm{~m}, 2 \mathrm{H}), 7.11-7.07$ (m, 2H), $5.61-5.32(\mathrm{~m}, 3 \mathrm{H}), 4.97(\mathrm{~s}, 1 \mathrm{H}), 4.41(\mathrm{~d}, J=16.4 \mathrm{~Hz}, 1 \mathrm{H}), 4.13(\mathrm{~d}, J$ $=16.4 \mathrm{~Hz}, 1 \mathrm{H}), 2.50(\mathrm{~s}, 3 \mathrm{H}) ;{ }^{13} \mathrm{C}$ NMR $(100 \mathrm{MHz}$, Chloroform- $d$ ) $\delta$ 153.4, 150.9, 150.6, 144.9, 144.3, 136.3, 133.6, 132.9, 132.4 (2C), 131.0 (2C), 130.4 (2C), 130.2 (2C), 127.6 (2C), 125.7, 125.3, 124.1 (2C), 123.4, 122.8, 122.2, 119.9, 119.5, 111.7, 63.5, 57.6, 43.6, 21.7; HRMS (ESI-TOF) m/z: [M-H] $]^{+}$calcd for $\left[\mathrm{C}_{32} \mathrm{H}_{24}{ }^{79} \mathrm{BrN}_{2} \mathrm{O}_{7} \mathrm{~S}_{2}\right]^{+}:$691.0203; found: 691.0216; calcd for $\left[\mathrm{C}_{32} \mathrm{H}_{24}{ }^{81} \mathrm{BrN}_{2} \mathrm{O}_{7} \mathrm{~S}_{2}\right]^{+}$: 693.0182; found: 693.0190. HPLC (Chiralpak IA, $n$-hexane/i-propanol $=7 / 3$, $1.0 \mathrm{~mL} / \mathrm{min}, 220 \mathrm{~nm}) \mathrm{t}_{\mathrm{R}}=12.78 \mathrm{~min}, 21.02 \mathrm{~min}$.

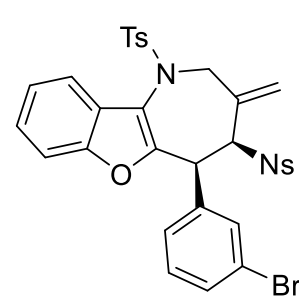

compound 3bk, white solid, $60.3 \mathrm{mg}$, yield $87 \%$, m.p.: $129-131{ }^{\circ} \mathrm{C} ;[\alpha]_{\mathrm{D}}{ }^{20}=$ $+11.1\left(\mathrm{CH}_{2} \mathrm{Cl}_{2}, c=1.00\right) ;{ }^{1} \mathbf{H}$ NMR $(400 \mathrm{MHz}$, Chloroform- $d$ ) $\delta 8.34(\mathrm{~d}, J=$ $8.4 \mathrm{~Hz}, 2 \mathrm{H}), 7.94(\mathrm{~d}, J=8.4 \mathrm{~Hz}, 2 \mathrm{H}), 7.62(\mathrm{~d}, J=7.9 \mathrm{~Hz}, 2 \mathrm{H}), 7.51-7.47(\mathrm{~m}$, 1H), $7.40-7.10(\mathrm{~m}, 9 \mathrm{H}), 5.55-5.20(\mathrm{~m}, 3 \mathrm{H}), 4.93(\mathrm{~s}, 1 \mathrm{H}), 4.44$ (d, $J=16.3$ $\mathrm{Hz}, 1 \mathrm{H}), 4.15(\mathrm{~d}, J=16.3 \mathrm{~Hz}, 1 \mathrm{H}), 2.50(\mathrm{~s}, 3 \mathrm{H}) ;{ }^{13} \mathbf{C} \mathbf{N M R}(100 \mathrm{MHz}$, Chloroform- $d$ ) $\delta 153.4,150.8,150.2,144.9,144.3,136.6,136.3,133.3,133.1,131.6,130.3$ (2C), 130.1 (2C), 129.8, 129.4, 127.5 (2C), 125.6, 125.3, 124.1 (2C), 123.4, 122.4, 121.7, 119.9, 119.5, 111.7, 64.0, 57.2, 43.7, 21.7; HRMS (ESI-TOF) m/z: $[\mathrm{M}-\mathrm{H}]^{+}$calcd for $\left[\mathrm{C}_{32} \mathrm{H}_{24}{ }^{79} \mathrm{BrN}_{2} \mathrm{O}_{7} \mathrm{~S}_{2}\right]^{+}$: 691.0203; found: 691.0206; calcd for $\left[\mathrm{C}_{32} \mathrm{H}_{24}{ }^{81} \mathrm{BrN}_{2} \mathrm{O}_{7} \mathrm{~S}_{2}\right]^{+}$: 693.0182; found: 693.0186. HPLC $\left(\right.$ Chiralpak AD, $n$-hexane $/ i$-propanol = 6/4, $1.0 \mathrm{~mL} / \mathrm{min}, 220 \mathrm{~nm}$ ) $\mathrm{t}_{\mathrm{R}}=11.78 \mathrm{~min}, 25.44 \mathrm{~min}$.

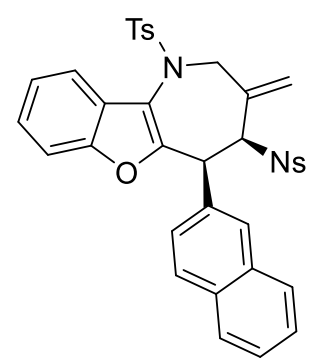

compound 3bl, white solid, $53.8 \mathrm{mg}$, yield $81 \%$, m.p.: $111-113{ }^{\circ} \mathrm{C} ;[\alpha]_{\mathrm{D}}{ }^{20}=$ $+19.0\left(\mathrm{CH}_{2} \mathrm{Cl}_{2}, c=1.00\right) ;{ }^{1} \mathrm{H}$ NMR $(400 \mathrm{MHz}$, Chloroform- $d) \delta 8.28(\mathrm{~d}, J=$ $8.3 \mathrm{~Hz}, 2 \mathrm{H}), 7.89(\mathrm{~d}, J=8.4 \mathrm{~Hz}, 2 \mathrm{H}), 7.85-7.71(\mathrm{~m}, 4 \mathrm{H}), 7.64(\mathrm{~d}, J=8.0$ $\mathrm{Hz}, 2 \mathrm{H}), 7.46(\mathrm{~m}, 2 \mathrm{H}), 7.35$ (d, $J=7.9 \mathrm{~Hz}, 2 \mathrm{H}), 7.33-7.12(\mathrm{~m}, 5 \mathrm{H}), 5.55-$ $5.38(\mathrm{~m}, 3 \mathrm{H}), 4.96(\mathrm{~s}, 1 \mathrm{H}), 4.47(\mathrm{~d}, J=16.4 \mathrm{~Hz}, 1 \mathrm{H}), 4.21(\mathrm{~d}, J=16.3 \mathrm{~Hz}$, 1H), 2.51 (s, 3H); ${ }^{13} \mathbf{C}$ NMR (100 MHz, Chloroform-d) $\delta$ 153.4, 151.2, 150.7, 144.8, 144.6, 136.4, 133.2, 132.8, 132.0, 130.3(3), 130.3(0) (3C), 130.1(2C), 128.2(0), 128.1(7), 127.7, 127.6 (2C), 127.2, 126.5, 126.2, 125.9, 125.1, 124.0 (2C), 123.4, 122.5, 119.9, 119.5, 111.7, 64.5, 57.5, 44.4, 21.7; HRMS (ESI-TOF) m/z: [M-H] $]^{+}$calcd for $\left[\mathrm{C}_{36} \mathrm{H}_{27} \mathrm{~N}_{2} \mathrm{O}_{7} \mathrm{~S}_{2}\right]^{+}: 663.1254$; found: 663.1259. HPLC (Chiralpak IA, $n$-hexane $i$-propanol $=7 / 3,1.0 \mathrm{~mL} / \mathrm{min}, 220 \mathrm{~nm}$ ) $\mathrm{t}_{\mathrm{R}}=16.23 \mathrm{~min}$, $22.14 \mathrm{~min}$. 


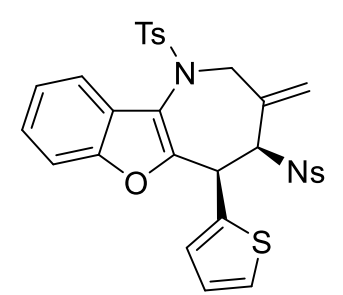

compound 3bm, white solid, $54.0 \mathrm{mg}$, yield 87\%, m.p.: $131-133{ }^{\circ} \mathrm{C} ;[\alpha]_{\mathrm{D}}{ }^{20}$ $=+15.4\left(\mathrm{CH}_{2} \mathrm{Cl}_{2}, c=1.00\right) ;{ }^{1} \mathbf{H}$ NMR $(400 \mathrm{MHz}$, Chloroform- $d) \delta 8.29(\mathrm{~d}$, $J=8.4 \mathrm{~Hz}, 2 \mathrm{H}), 7.88(\mathrm{~d}, J=8.4 \mathrm{~Hz}, 2 \mathrm{H}), 7.60(\mathrm{~d}, J=7.9 \mathrm{~Hz}, 2 \mathrm{H}), 7.41-$ $7.15(\mathrm{~m}, 8 \mathrm{H}), 7.02-6.94(\mathrm{~m}, 1 \mathrm{H}), 5.67-5.30(\mathrm{~m}, 3 \mathrm{H}), 4.70-4.53(\mathrm{~m}, 2 \mathrm{H})$, $4.23(\mathrm{~d}, J=16.2 \mathrm{~Hz}, 1 \mathrm{H}), 2.45(\mathrm{~s}, 3 \mathrm{H}) ;{ }^{13} \mathrm{C}$ NMR (100 MHz, Chloroform- $d$ ) $\delta 153.4,150.7,150.1,144.7,144.6,136.1,135.8,134.3,130.1,130.0$ (3C), 129.7, 127.6 (2C), 126.6, 125.8, 125.6, 125.2, 124.0 (2C), 123.8, 123.5, 120.1, 118.9, 111.6, 66.7, 56.2, 39.9, 21.6; HRMS (ESI-TOF) m/z: $[\mathrm{M}-\mathrm{H}]^{+}$calcd for $\left[\mathrm{C}_{30} \mathrm{H}_{24} \mathrm{~N}_{2} \mathrm{O}_{7} \mathrm{~S}_{3}\right]^{+}:$619.0662; found: 619.0665. HPLC (Chiralpak IA, $n$-hexane $/ i$-propanol $=6 / 4,1.0 \mathrm{~mL} / \mathrm{min}, 220 \mathrm{~nm}) \mathrm{t}_{\mathrm{R}}=14.99 \mathrm{~min}, 31.36 \mathrm{~min}$.

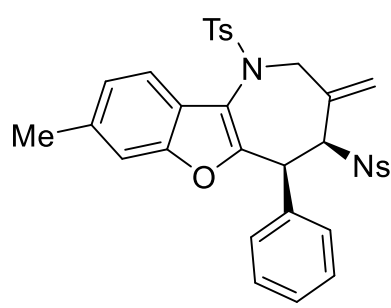

compound 3bn, white solid, $52.8 \mathrm{mg}$, yield 84\%, m.p.: $134-135{ }^{\circ} \mathrm{C}$; $[\alpha] \mathrm{D}^{20}=-20.1\left(\mathrm{CH}_{2} \mathrm{Cl}_{2}, c=1.00\right) ;{ }^{1} \mathbf{H}$ NMR $(400 \mathrm{MHz}$, Chloroform- $d) \delta$ $8.39-8.27(\mathrm{~m}, 2 \mathrm{H}), 7.93(\mathrm{~d}, J=8.7 \mathrm{~Hz}, 2 \mathrm{H}), 7.66-7.60(\mathrm{~m}, 2 \mathrm{H}), 7.41-$ $7.27(\mathrm{~m}, 5 \mathrm{H}), 7.27-7.18(\mathrm{~m}, 2 \mathrm{H}), 7.11(\mathrm{~s}, 1 \mathrm{H}), 7.08-7.01(\mathrm{~m}, 1 \mathrm{H})$, $7.02-6.95(\mathrm{~m}, 1 \mathrm{H}), 5.52-5.29(\mathrm{~m}, 3 \mathrm{H}), 4.90(\mathrm{~s}, 1 \mathrm{H}), 4.42(\mathrm{~d}, J=16.4$ $\mathrm{Hz}, 1 \mathrm{H}), 4.15(\mathrm{~d}, J=16.4 \mathrm{~Hz}, 1 \mathrm{H}), 2.51(\mathrm{~s}, 3 \mathrm{H}), 2.40(\mathrm{~s}, 3 \mathrm{H}) ;{ }^{13} \mathbf{C} \mathbf{~ N M R}$ (100 MHz, Chloroform-d) $\delta 153.8,150.8,150.3,144.7,144.6,136.5,135.5,134.6,133.1,130.8$ (2C), 130.3 (2C), 130.1 (2C), 128.4, 127.7 (2C), 127.5 (2C), 124.7, 124.0 (2C), 123.3, 122.1, 119.4, 119.3, 111.8, 64.1, 57.6, 44.2, 21.7, 21.6; HRMS (ESI-TOF) m/z: [M-H] $]^{+}$calcd for $\left[\mathrm{C}_{33} \mathrm{H}_{27} \mathrm{~N}_{2} \mathrm{O}_{7} \mathrm{~S}_{2}\right]^{+}$: 627.1254; found: 627.1262. HPLC (Chiralpak IA, $n$-hexane/i-propanol = 7/3, $1.0 \mathrm{~mL} / \mathrm{min}, 220 \mathrm{~nm}$ ) $\mathrm{t}_{\mathrm{R}}=17.50 \mathrm{~min}, 38.77 \mathrm{~min}$.

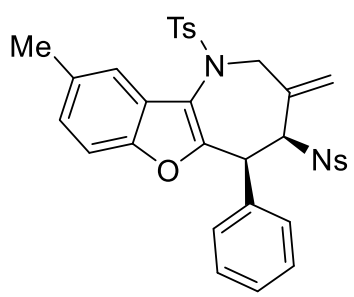

compound 3bo, white solid, $54.1 \mathrm{mg}$, yield $86 \%$, m.p.: $146-148{ }^{\circ} \mathrm{C} ;[\alpha]_{\mathrm{D}}{ }^{20}$ $=+16.3\left(\mathrm{CH}_{2} \mathrm{Cl}_{2}, c=1.00\right) ;{ }^{1} \mathbf{H}$ NMR $(400 \mathrm{MHz}$, Chloroform- $d) \delta 8.33(\mathrm{~d}$, $J=8.5 \mathrm{~Hz}, 2 \mathrm{H}), 7.93(\mathrm{~d}, J=8.4 \mathrm{~Hz}, 2 \mathrm{H}), 7.63(\mathrm{~d}, J=7.9 \mathrm{~Hz}, 2 \mathrm{H}), 7.39-$ $7.12(\mathrm{~m}, 8 \mathrm{H}), 7.01(\mathrm{~m}, 1 \mathrm{H}), 6.75(\mathrm{~s}, 1 \mathrm{H}), 5.53-5.29(\mathrm{~m}, 3 \mathrm{H}), 4.94(\mathrm{~s}, 1 \mathrm{H})$, $4.49(\mathrm{~d}, J=16.4 \mathrm{~Hz}, 1 \mathrm{H}), 4.14(\mathrm{~d}, J=16.3 \mathrm{~Hz}, 1 \mathrm{H}), 2.50(\mathrm{~s}, 3 \mathrm{H}), 2.29$ (s, 3H); ${ }^{13}$ C NMR (100 MHz, Chloroform-d) $\delta 151.8,151.3,150.7,144.7,144.6,136.5,134.5,133.2$, 132.7, 130.8 (2C), 130.3 (2C), 130.0 (2C), 128.4, 127.8 (2C), 127.7 (2C), 126.3, 125.7, 124.0 (2C), 122.1, 119.5, 119.0, 111.2, 64.2, 57.6, 44.3, 21.7, 21.2; HRMS (ESI-TOF) m/z: [M-H] ${ }^{+}$calcd for $\left[\mathrm{C}_{32} \mathrm{H}_{27} \mathrm{~N}_{2} \mathrm{O}_{7} \mathrm{~S}_{2}\right]^{+}$: 627.1254; found: 627.1259. HPLC (Chiralpak IA, $n$-hexane/i-propanol = 7/3, 1.0 $\mathrm{mL} / \mathrm{min}, 220 \mathrm{~nm}) \mathrm{t}_{\mathrm{R}}=10.87 \mathrm{~min}, 12.50 \mathrm{~min}$.

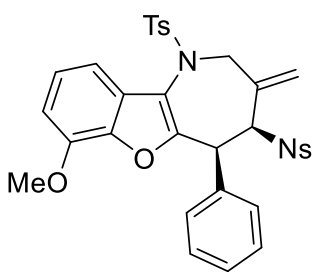

compound 3bp, white solid, $53.5 \mathrm{mg}$, yield $83 \%$, m.p.: $143-145{ }^{\circ} \mathrm{C}$; $[\alpha]_{\mathrm{D}}{ }^{20}$ $=-9.0\left(\mathrm{CH}_{2} \mathrm{Cl}_{2}, c=1.00\right) ;{ }^{1} \mathbf{H}$ NMR $(400 \mathrm{MHz}$, Chloroform- $d) \delta 8.32(\mathrm{~d}, J$ $=8.8 \mathrm{~Hz}, 2 \mathrm{H}), 7.92(\mathrm{~d}, J=8.6 \mathrm{~Hz}, 2 \mathrm{H}), 7.62(\mathrm{~d}, J=8.2 \mathrm{~Hz}, 2 \mathrm{H}), 7.38-$ $7.25(\mathrm{~m}, 5 \mathrm{H}), 7.23-7.17(\mathrm{~m}, 2 \mathrm{H}), 7.10-7.02(\mathrm{~m}, 1 \mathrm{H}), 6.81-6.71(\mathrm{~m}, 2 \mathrm{H})$, 
$5.50-5.34(\mathrm{~m}, 3 \mathrm{H}), 4.89(\mathrm{~s}, 1 \mathrm{H}), 4.43(\mathrm{~d}, J=16.3 \mathrm{~Hz}, 1 \mathrm{H}), 4.16(\mathrm{~d}, J=16.4 \mathrm{~Hz}, 1 \mathrm{H}), 3.85(\mathrm{~s}, 3 \mathrm{H})$, 2.49 (s, 3H); ${ }^{13}$ C NMR (100 MHz, Chloroform- $d$ ) $\delta$ 151.2, 150.7, 145.2, 144.7, 144.6, 142.8, 136.4, 134.3, 133.1, 130.8 (2C), 130.4 (2C), 130.1 (2C), 128.4, 127.7 (2C), 127.6 (2C), 127.5, 124.0 (2C), 123.9, 122.3, 119.8, 112.0, 107.4, 64.4, 57.4, 56.1, 44.2, 21.7; HRMS (ESI-TOF) m/z: [M-H] ${ }^{+}$calcd for $\left[\mathrm{C}_{33} \mathrm{H}_{27} \mathrm{~N}_{2} \mathrm{O}_{8} \mathrm{~S}_{2}\right]^{+}:$643.1203; found: 643.1208. HPLC (Chiralpak AD, $n$-hexane/i-propanol $=7 / 3$, $1.0 \mathrm{~mL} / \mathrm{min}, 220 \mathrm{~nm}) \mathrm{t}_{\mathrm{R}}=17.97 \mathrm{~min}, 54.07 \mathrm{~min}$.

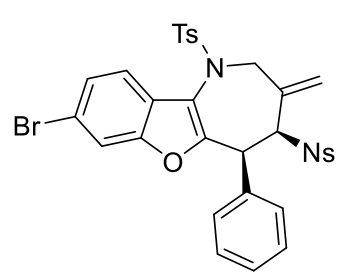

compound 3bq, white solid, $48.6 \mathrm{mg}$, yield $70 \%$, m.p.: $128-130{ }^{\circ} \mathrm{C} ;[\alpha]_{\mathrm{D}}{ }^{20}$ $=-11.0\left(\mathrm{CH}_{2} \mathrm{Cl}_{2}, c=1.00\right) ;{ }^{1} \mathbf{H}$ NMR $(400 \mathrm{MHz}$, Chloroform- $d) \delta 8.34(\mathrm{~d}, J$ $=8.8 \mathrm{~Hz}, 2 \mathrm{H}), 7.94-7.87(\mathrm{~m}, 2 \mathrm{H}), 7.63-7.57(\mathrm{~m}, 2 \mathrm{H}), 7.51-7.47(\mathrm{~m}$, $1 \mathrm{H}), 7.39-7.26(\mathrm{~m}, 6 \mathrm{H}), 7.21-7.17(\mathrm{~m}, 2 \mathrm{H}), 7.06-7.03(\mathrm{~m}, 1 \mathrm{H}), 5.46-$ $5.24(\mathrm{~m}, 3 \mathrm{H}), 4.83(\mathrm{~s}, 1 \mathrm{H}), 4.43(\mathrm{~d}, J=16.3 \mathrm{~Hz}, 1 \mathrm{H}), 4.15(\mathrm{~d}, J=16.3 \mathrm{~Hz}$,

1H), 2.51 (s, 3H); ${ }^{13}$ C NMR (100 MHz, Chloroform- $d$ ) $\delta$ 153.5, 151.8, 150.8, 145.0, 144.5, 136.2, 134.1, 132.9, 130.7 (2C), 130.3 (2C), 130.2 (2C), 128.7, 127.9 (2C), 127.6 (2C), 126.8, 125.0, 124.1 (2C), 122.6, 121.0, 119.4, 118.4, 115.1, 64.0, 57.6, 44.3, 21.7; HRMS (ESI-TOF) m/z: [M-H] $]^{+}$calcd for $\left[\mathrm{C}_{32} \mathrm{H}_{24}{ }^{79} \mathrm{BrN}_{2} \mathrm{O}_{7} \mathrm{~S}_{2}\right]^{+}$: 691.0203 ; found: 691.0212; calcd for $\left[\mathrm{C}_{32} \mathrm{H}_{24}{ }^{81} \mathrm{BrN}_{2} \mathrm{O}_{7} \mathrm{~S}_{2}\right]^{+}:$693.0182; found: 693.0190. HPLC (Chiralpak IA, $n$-hexane $/ i$-propanol $=7 / 3,1.0 \mathrm{~mL} / \mathrm{min}, 220 \mathrm{~nm}$ ) $\mathrm{t}_{\mathrm{R}}=14.37$ $\min , 19.32 \mathrm{~min}$.

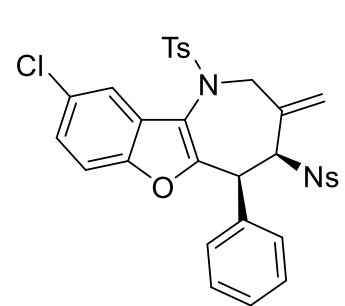

compound 3br, white solid, $52.0 \mathrm{mg}$, yield $80 \%$, m.p.: $154-155^{\circ} \mathrm{C} ;[\alpha] \mathrm{D}^{20}$ $=+13.2\left(\mathrm{CH}_{2} \mathrm{Cl}_{2}, c=1.00\right) ;{ }^{1} \mathbf{H}$ NMR $(400 \mathrm{MHz}$, Chloroform-d) $\delta 8.35(\mathrm{~d}$, $J=8.4 \mathrm{~Hz}, 2 \mathrm{H}), 7.96(\mathrm{~d}, J=8.4 \mathrm{~Hz}, 2 \mathrm{H}), 7.61(\mathrm{~d}, J=7.9 \mathrm{~Hz}, 2 \mathrm{H}), 7.42-$ $7.29(\mathrm{~m}, 5 \mathrm{H}), 7.28-7.12(\mathrm{~m}, 4 \mathrm{H}), 6.80(\mathrm{~s}, 1 \mathrm{H}), 5.53-5.34(\mathrm{~m}, 3 \mathrm{H}), 5.05$ - $4.96(\mathrm{~m}, 1 \mathrm{H}), 4.52(\mathrm{~d}, J=16.4 \mathrm{~Hz}, 1 \mathrm{H}), 4.13(\mathrm{~d}, J=16.4 \mathrm{~Hz}, 1 \mathrm{H}), 2.51$ (s, 3H); ${ }^{13}$ C NMR (100 MHz, Chloroform- $d$ ) $\delta$ 153.1, 151.6, 150.8, 145.2, 144.5, 136.1, 134.2, 133.0, 130.7 (2C), 130.3 (2C), 130.2 (2C), 129.1, 128.6, 127.9 (2C), 127.8 (2C), 127.0, 125.3, 124.1 (2C), 122.4, 119.4, 118.9, 112.7, 64.1, 57.4, 44.3, 21.7; HRMS (ESI-TOF) m/z: [M-H] ${ }^{+}$calcd for $\left[\mathrm{C}_{32} \mathrm{H}_{24} \mathrm{ClN}_{2} \mathrm{O}_{7} \mathrm{~S}_{2}\right]^{+}:$647.0708; found: 647.0711. HPLC (Chiralpak AD, $n$-hexane/i-propanol $=6 / 4$, $1.0 \mathrm{~mL} / \mathrm{min}, 220 \mathrm{~nm}) \mathrm{t}_{\mathrm{R}}=9.27 \mathrm{~min}, 23.65 \mathrm{~min}$.

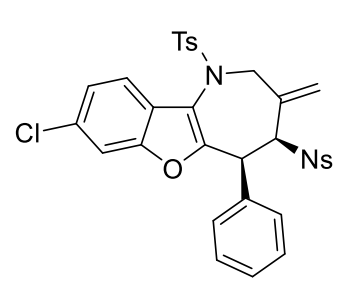

compound 3bs, white solid, $57.8 \mathrm{mg}$, yield $89 \%$, m.p.: $151-152{ }^{\circ} \mathrm{C} ;[\alpha]_{\mathrm{D}}{ }^{20}$ $=-32.0\left(\mathrm{CH}_{2} \mathrm{Cl}_{2}, c=1.00\right) ;{ }^{\mathbf{1}} \mathbf{H}$ NMR $(400 \mathrm{MHz}$, Chloroform- $d) \delta 8.33(\mathrm{~d}, J$ $=8.6 \mathrm{~Hz}, 2 \mathrm{H}), 7.90(\mathrm{~d}, J=8.5 \mathrm{~Hz}, 2 \mathrm{H}), 7.60(\mathrm{~d}, J=8.0 \mathrm{~Hz}, 2 \mathrm{H}), 7.41-$ $7.29(\mathrm{~m}, 6 \mathrm{H}), 7.23-7.18(\mathrm{~m}, 2 \mathrm{H}), 7.17-7.05(\mathrm{~m}, 2 \mathrm{H}), 5.43-5.33(\mathrm{~m}, 3 \mathrm{H})$, $4.82(\mathrm{~m}, 1 \mathrm{H}), 4.43(\mathrm{~d}, J=16.3 \mathrm{~Hz}, 1 \mathrm{H}), 4.16(\mathrm{~d}, J=16.3 \mathrm{~Hz}, 1 \mathrm{H}), 2.51(\mathrm{~s}$, $3 \mathrm{H}) ;{ }^{13} \mathrm{C}$ NMR (100 MHz, Chloroform- $d$ ) $\delta$ 153.3, 151.9, 150.8, 145.0, 144.5, 136.2, 134.2, 132.9, 131.0, 130.7 (2C), 130.3 (2C), 130.2 (2C), 128.6, 127.9 (2C), 127.6 (2C), 124.5, 124.2, 124.1 (2C), 
122.6, 120.6, 119.3, 112.2, 64.1, 57.4, 44.3, 21.7; HRMS (ESI-TOF) m/z: $[\mathrm{M}-\mathrm{H}]^{+}$calcd for $\left[\mathrm{C}_{32} \mathrm{H}_{24} \mathrm{ClN}_{2} \mathrm{O}_{7} \mathrm{~S}_{2}\right]^{+}:$647.0708; found: 647.0714. HPLC (Chiralpak IA, $n$-hexane/i-propanol = 7/3, 1.0 $\mathrm{mL} / \mathrm{min}, 220 \mathrm{~nm}) \mathrm{t}_{\mathrm{R}}=13.34 \mathrm{~min}, 19.13 \mathrm{~min}$.

\section{General Procedure for desulfonylation}
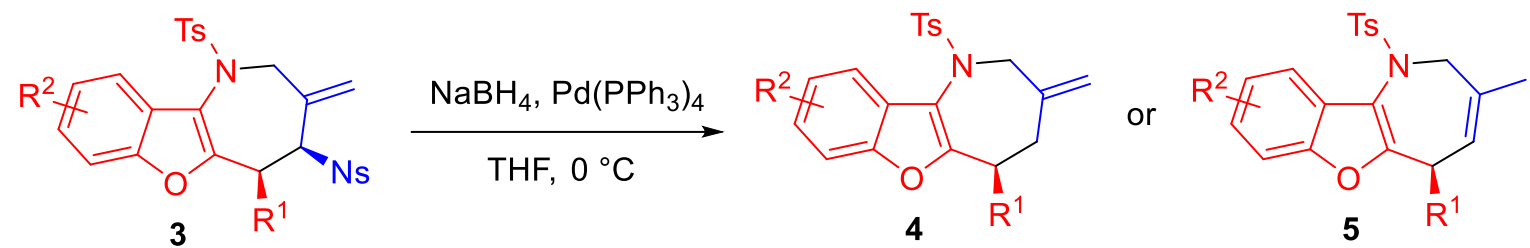

Under a nitrogen atmosphere, $3(0.1 \mathrm{mmol})$ and $\mathrm{Pd}\left(\mathrm{PPh}_{3}\right)_{4}(11.6 \mathrm{mg}, 0.01 \mathrm{mmol})$ were dissolved in THF $(2.0 \mathrm{~mL})$, and stirred at $0{ }^{\circ} \mathrm{C}$ for approximately $10 \mathrm{~min}$. Then $\mathrm{NaBH}_{4}(7.6 \mathrm{mg}, 0.2 \mathrm{mmol})$ was added. Once starting material was consumed (monitored by TLC), $2 \mathrm{~mL}$ ice water was added to quench the reaction. The organic and aqueous layers were separated. The aqueous layer was extracted with ethyl acetate (10 $\mathrm{mL} \mathrm{X3} \mathrm{times).} \mathrm{The} \mathrm{combined} \mathrm{organic} \mathrm{layer} \mathrm{was} \mathrm{washed} \mathrm{with} \mathrm{brine,}$ dried over $\mathrm{Na}_{2} \mathrm{SO}_{4}$, after evaporation of solvent, the crude product was purified by column chromatography (petroleum ether/ethyl acetate $=6: 1$ ) on silica gel to afford 4 or 5 .

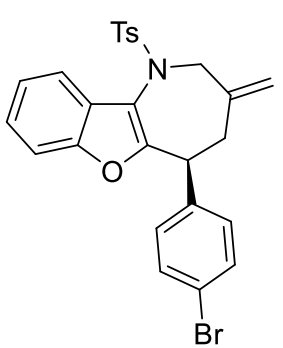

compound 4a, white solid, $30.4 \mathrm{mg}$, yield $60 \%$, m.p.: $121-123{ }^{\circ} \mathrm{C} ;[\alpha]_{\mathrm{D}}^{20}=$ $+83.5\left(\mathrm{CH}_{2} \mathrm{Cl}_{2}, c=1.00\right) ;{ }^{1} \mathbf{H}$ NMR (400 MHz, Chloroform-d) $\delta 7.71-7.65$ $(\mathrm{m}, 3 \mathrm{H}), 7.42-7.34(\mathrm{~m}, 2 \mathrm{H}), 7.29-7.21(\mathrm{~m}, 5 \mathrm{H}), 6.85(\mathrm{~d}, \mathrm{~J}=8.2 \mathrm{~Hz}, 2 \mathrm{H})$, $5.01-4.87$ (m, 2H), 4.77 - 4.60 (brs, 1H), $4.48-4.40$ (brs, 1H), $3.96-3.77$ (brs, 1H), 2.42 (s, 3H), $2.39-2.33(\mathrm{~m}, 1 \mathrm{H}), 2.28-2.12(\mathrm{~m}, 1 \mathrm{H}) ;{ }^{13} \mathbf{C}$ NMR (100 MHz, Chloroform-d) $\delta 153.4,152.7,144.0,142.4,140.0,136.3,131.6$

(2C), 129.7 (2C), 129.4 (2C), 127.9 (2C), 126.5, 124.7, 123.1, 121.2, 121.0, 119.9, 115.0, 111.2, 57.4, 45.5, 37.8, 21.6 ; HRMS (EI-TOF) $\mathrm{m} / \mathrm{z}$ : $[\mathrm{M}]^{+}$calcd for $\left[\mathrm{C}_{26} \mathrm{H}_{22}{ }^{79} \mathrm{BrNO}_{3} \mathrm{~S}\right]^{+}$: 507.0498 ; found: 507.0503; calcd for $\left[\mathrm{C}_{26} \mathrm{H}_{22}{ }^{81} \mathrm{BrNO}_{3} \mathrm{~S}\right]^{+}$: 509.0478; found: 509.0484. HPLC (Chiralpak IF, $n$ hexane/ethanol $=98 / 2,1.0 \mathrm{~mL} / \mathrm{min}, 220 \mathrm{~nm}$ ) $\mathrm{t}_{\mathrm{R}}=27.67 \mathrm{~min}, 39.10 \mathrm{~min}$.

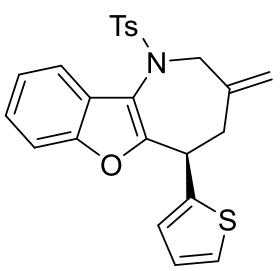

compound 4b, white solid, $23.1 \mathrm{mg}$, yield 53\%, m.p.: $115-116{ }^{\circ} \mathrm{C} ;[\alpha]_{\mathrm{D}}{ }^{20}=$ $+96.4\left(\mathrm{CH}_{2} \mathrm{Cl}_{2}, c=1.00\right) ;{ }^{1} \mathrm{H}$ NMR $(400 \mathrm{MHz}$, Chloroform- $d) \delta 7.60(\mathrm{~d}, J=$ $8.0 \mathrm{~Hz}, 2 \mathrm{H}), 7.52-7.40(\mathrm{~m}, 1 \mathrm{H}), 7.38-7.33(\mathrm{~m}, 1 \mathrm{H}), 7.31-7.27(\mathrm{~m}, 2 \mathrm{H})$, $7.20-7.14(\mathrm{~m}, 3 \mathrm{H}), 6.96-6.87(\mathrm{~m}, 1 \mathrm{H}), 6.69-6.62(\mathrm{~m}, 1 \mathrm{H}), 5.00(\mathrm{~s}, 1 \mathrm{H})$, $4.91(\mathrm{~s}, 1 \mathrm{H}), 4.58-4.42(\mathrm{~m}, 2 \mathrm{H}), 4.19-4.08$ (brs, 1H), $2.45-2.37$ (m, 4H), $2.25-2.07$ (brs, 1H); ${ }^{13} \mathbf{C}$ NMR (100 MHz, Chloroform- $d$ ) $\delta$ 153.3, 152.1, 143.9, 141.9, 135.6, 133.3, 133.2, 129.6 (2C), 127.8 (2C), 126.6, 126.5, 124.8, 124.7, 124.2, 123.2, 121.5, 115.8, 111.2, 57.5, 40.8, 37.7, 21.6; HRMS (EI-TOF) m/z: [M] calcd for $\left[\mathrm{C}_{24} \mathrm{H}_{21} \mathrm{NO}_{3} \mathrm{~S}_{2}\right]^{+}$: 435.0957; found: 435.0962; HPLC (Chiralpak AD, $n$-hexane $/ i$-propanol $=90 / 10,1.0 \mathrm{~mL} / \mathrm{min}, 220 \mathrm{~nm}) \mathrm{t}_{\mathrm{R}}=9.84 \mathrm{~min}, 11.80 \mathrm{~min}$. 


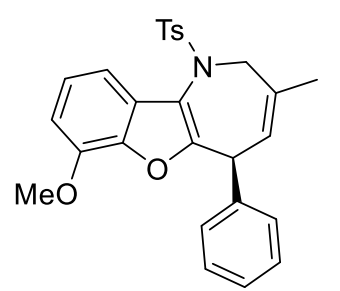

compound 5, white solid, $28.0 \mathrm{mg}$, yield 57\%, m.p.: $118-120{ }^{\circ} \mathrm{C} ;[\alpha]_{\mathrm{D}}{ }^{20}=-$ $90.5\left(\mathrm{CH}_{2} \mathrm{Cl}_{2}, c=1.00\right)$; ${ }^{1} \mathbf{H}$ NMR (400 MHz, Chloroform- $\left.d\right) \delta 7.54(\mathrm{~d}, J=$ $8.0 \mathrm{~Hz}, 2 \mathrm{H}), 7.46(\mathrm{~d}, J=8.0 \mathrm{~Hz}, 1 \mathrm{H}), 7.24-7.14(\mathrm{~m}, 6 \mathrm{H}), 6.93-6.86(\mathrm{~m}$, $2 \mathrm{H}), 6.76(\mathrm{~d}, J=7.9 \mathrm{~Hz}, 1 \mathrm{H}), 5.18-5.11$ (brs, $1 \mathrm{H}), 4.50-4.41$ (brs, $1 \mathrm{H})$, $4.36-4.28$ (m, 2H), 3.88 (s, 3H), 2.43 (s, 3H), 1.82 (s, 3H); ${ }^{13}$ C NMR (100 MHz, Chloroform- $d$ ) $\delta$ 150.7, 144.8, 143.7, 142.6, 140.9, 136.0, 132.4, 129.3 (2C), 128.6 (2C), 128.1(8) (2C), 128.1(6), 127.9 (2C), 127.0, 124.2, 123.7, 121.0, 113.7, 106.9, 56.0, 53.1, 46.4, 26.0, 21.7.; HRMS (EI-TOF) m/z: [M] ${ }^{+}$calcd for $\left[\mathrm{C}_{27} \mathrm{H}_{25} \mathrm{NO}_{4} \mathrm{~S}\right]^{+}$: 459.1499; found: 459.1506; HPLC $($ Chiralpak IF, $n$-hexane $/ i$-propanol $=8 / 2,1.0 \mathrm{~mL} / \mathrm{min}, 220 \mathrm{~nm}) \mathrm{t}_{\mathrm{R}}=11.64 \mathrm{~min}, 13.00 \mathrm{~min}$.

\section{Gram scale procedure for the asymmetric $[4+3]$ cyclization of TMM donor $1 \mathrm{~b}$ with benzofuran-derived azadiene $2 j$}
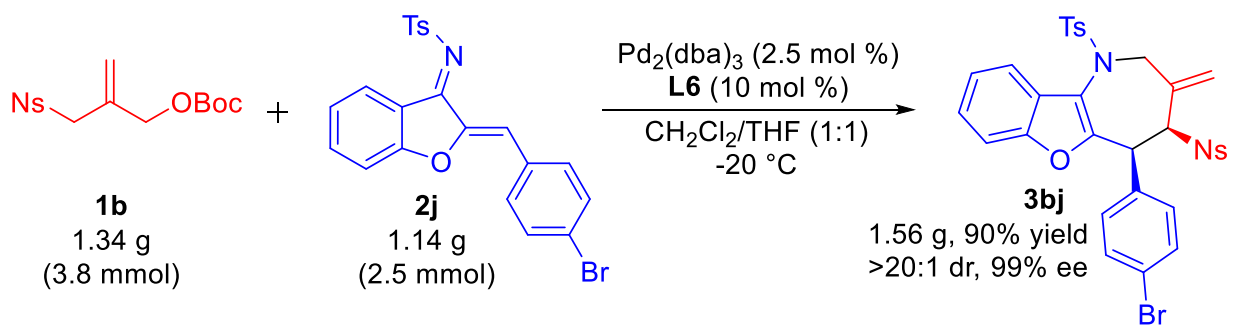

Under a nitrogen atmosphere, $\mathrm{Pd}_{2}(\mathrm{dba})_{3}(57.2 \mathrm{mg}, 0.0625 \mathrm{mmol})$ and ligand $\mathbf{L 6}(128.9 \mathrm{mg}, 0.25$ $\mathrm{mmol})$ were dissolved in DCM/THF $=1: 1(\mathrm{v} / \mathrm{v}=1: 1,25.0 \mathrm{~mL})$, and stirred at room temperature for approximately $30 \mathrm{~min}$. Then the mixture was cooled to $-20{ }^{\circ} \mathrm{C}$ and sulfonyl-TMM donor $\mathbf{1 b}(1.34 \mathrm{~g}$, $3.8 \mathrm{mmol})$ and benzofuran-derived azadiene $2 \mathrm{j}(1.14 \mathrm{~g}, 2.5 \mathrm{mmol})$ were added sequentially. Once starting material was consumed (monitored by TLC), the solvent was evaporated. The mixture was concentrated and purified by column chromatography to give the cycloaddition product $\mathbf{3 b j}$ (1.56 g, $90 \%$ yield).

\section{Procedure for the deprotection of 4 a to 6}

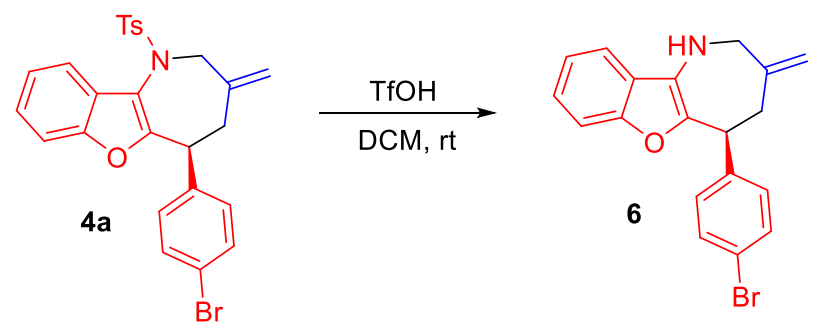

To a solution of $\mathbf{4 a}(50.7 \mathrm{mg}, 0.1 \mathrm{mmol})$ in dichloromethane $(1 \mathrm{~mL}, 0.1 \mathrm{M})$, TfOH $(18 \mu \mathrm{L}, 0.2 \mathrm{mmol}$, 2 equiv.) was added. The reaction mixture was stirred at room temperature for 12 hours under nitrogen atmosphere. Then, saturated aqueous $\mathrm{NaHCO}_{3}$ solution was added and the mixture was extracted with dichloromethane three times. The combined organic layers were dried over anhydrous $\mathrm{Na}_{2} \mathrm{SO}_{4}$, filtered and evaporated. Finally, solvents were removed and the crude mixture was purified by flash chromatography with 6:1 petroleum ether: ethyl acetate as eluent to afford 6 ( $24.7 \mathrm{mg}, 70 \%$ 
yield) as a yellow solid.

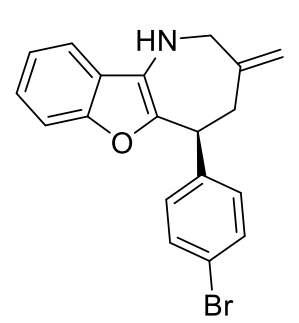

compound 6, yellow solid, $24.7 \mathrm{mg}$, yield 70\%, m.p.: $112-114{ }^{\circ} \mathrm{C} ;[\alpha]_{\mathrm{D}}{ }^{20}=$ $131.2\left(\mathrm{CH}_{2} \mathrm{Cl}_{2}, c=1.00\right) ;{ }^{1} \mathbf{H}$ NMR $\left(400 \mathrm{MHz}\right.$, Acetone- $\left.d_{6}\right) \delta 7.66-7.58(\mathrm{~m}$, $1 \mathrm{H}), 7.49-7.38(\mathrm{~m}, 2 \mathrm{H}), 7.26-7.14(\mathrm{~m}, 5 \mathrm{H}), 4.94(\mathrm{~s}, 1 \mathrm{H}), 4.80(\mathrm{~s}, 1 \mathrm{H}), 4.67$ (s, 1H), $4.46(\mathrm{dd}, J=6.3,5.0 \mathrm{~Hz}, 1 \mathrm{H}), 3.92(\mathrm{~d}, J=13.2 \mathrm{~Hz}, 1 \mathrm{H}), 3.79$ (d, $J=$ $13.1 \mathrm{~Hz}, 1 \mathrm{H}), 2.99$ (dd, $J=13.2,5.1 \mathrm{~Hz}, 1 \mathrm{H}), 2.78(\mathrm{dd}, J=13.2,6.4 \mathrm{~Hz}, 1 \mathrm{H})$;

${ }^{13}$ C NMR (100 MHz, Acetone- $\left.d_{6}\right) \delta$ 153.6, 146.2, 143.7, 140.7, 131.9 (2C), 131.0 (2C), 130.1, 127.1, 126.0, 124.8, 122.4, 118.9, 115.4, 111.4, 55.0, 45.1, 42.2; HRMS (EI-TOF) m/z: $[\mathrm{M}]^{+}$calcd for $\left[\mathrm{C}_{19} \mathrm{H}_{16}{ }^{79} \mathrm{BrNO}\right]^{+}$: 353.0410 ; found: 353.0401 ; calcd for $\left[\mathrm{C}_{19} \mathrm{H}_{16}{ }^{81} \mathrm{BrNO}\right]^{+}$: 355.0389; found: 355.0408; HPLC (Chiralpak AD, $n$-hexane $/ i$-propanol = 94/6, $1.0 \mathrm{~mL} / \mathrm{min}, 220 \mathrm{~nm}$ ) $\mathrm{t}_{\mathrm{R}}=17.22 \mathrm{~min}, 21.81 \mathrm{~min}$. 


\section{The absolute configuration determination of $(4 S, 5 S)-3 \mathrm{bq}$}

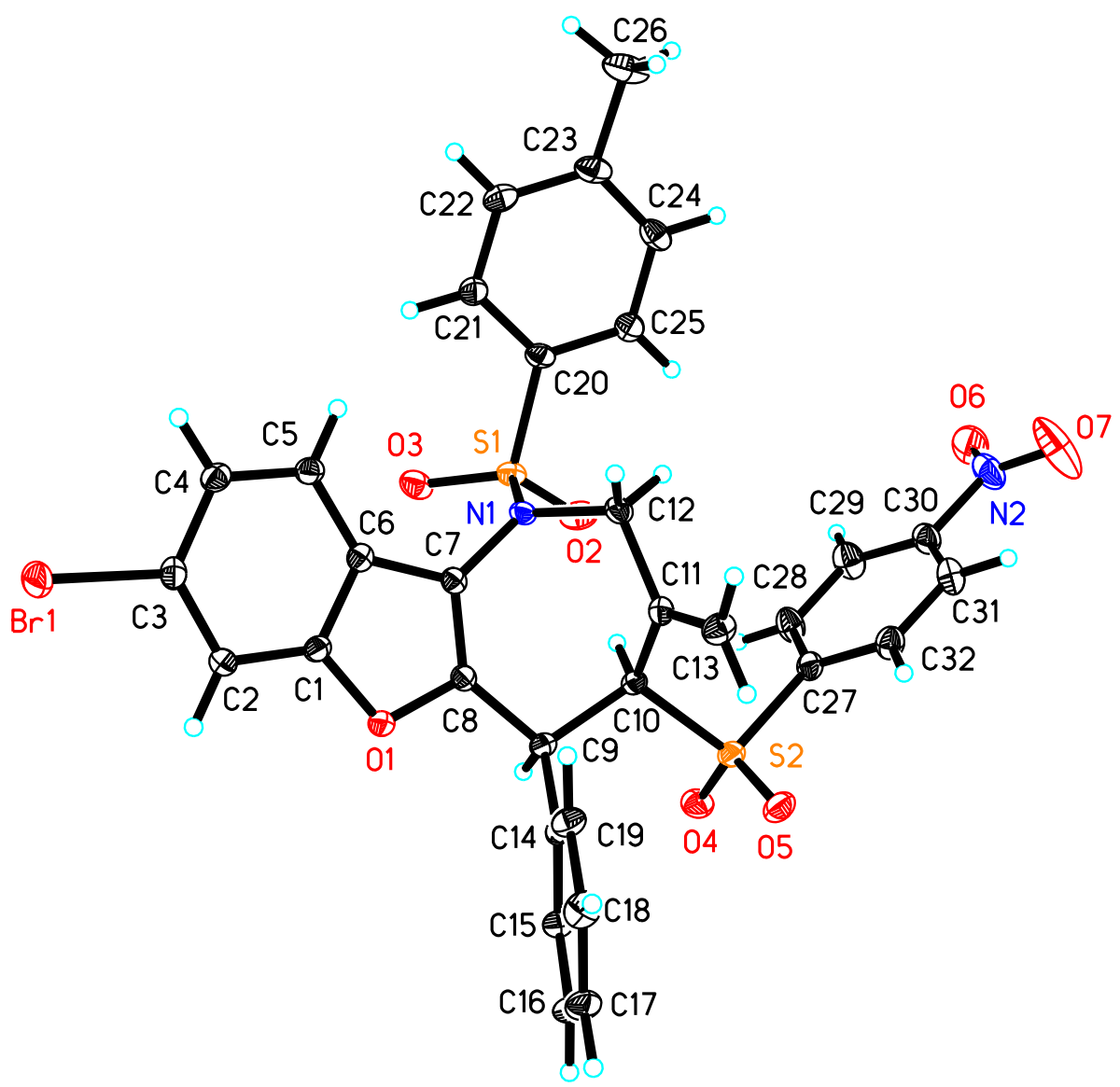

Fig S2. X-ray structure of $(4 S, 5 S)$-3bq. Ellipsoids are drawn at the $30 \%$ probability level.

\section{Sample Preparation}

Add $20 \mathrm{mg}$ substrate and $2 \mathrm{~mL}$ acetone to a $4 \mathrm{~mL}$ vial, and slowly evaporate solvent to grow crystal at room temperature

\section{Crystal data and structure refinement for CCDC 2048560}

Identification code

Empirical formula

Formula weight

Temperature

Wavelength

Crystal system mo_d8v19882_0m

C35 H31 Br N2 O8 S2

751.65

193(2) K

$0.71073 \AA$

Orthorhombic 
Space group

Unit cell dimensions

Volume

Z

Density (calculated)

Absorption coefficient

$\mathrm{F}(000)$

Crystal size

Theta range for data collection

Index ranges

Reflections collected

Independent reflections

Completeness to theta $=25.242^{\circ}$

Absorption correction

Max. and min. transmission

Refinement method

Data / restraints / parameters

Goodness-of-fit on $\mathrm{F}^{2}$

Final R indices [I $>2 \operatorname{sigma}(\mathrm{I})]$

$\mathrm{R}$ indices (all data)

Absolute structure parameter

Extinction coefficient

Largest diff. peak and hole
P 212121

$$
\begin{array}{ll}
\mathrm{a}=11.6303(4) \AA & \alpha=90^{\circ} . \\
\mathrm{b}=13.9514(5) \AA & \beta=90^{\circ} . \\
\mathrm{c}=20.4059(7) \AA & \gamma=90^{\circ} .
\end{array}
$$

$$
3311.0(2) \AA^{3}
$$

4

$1.508 \mathrm{Mg} / \mathrm{m}^{3}$

$1.424 \mathrm{~mm}^{-1}$

1544

$0.170 \times 0.150 \times 0.120 \mathrm{~mm}^{3}$

2.473 to $26.000^{\circ}$.

$-14<=\mathrm{h}<=14,-17<=\mathrm{k}<=17,-24<=\mathrm{l}<=25$

49371

$6483[\mathrm{R}(\mathrm{int})=0.0431]$

$99.6 \%$

Semi-empirical from equivalents

0.7456 and 0.4311

Full-matrix least-squares on $\mathrm{F}^{2}$

6483 / 0 / 437

1.029

$\mathrm{R} 1=0.0285, \mathrm{wR} 2=0.0716$

$\mathrm{R} 1=0.0325, \mathrm{wR} 2=0.0742$

$0.019(3)$

$0.0028(5)$

0.887 and -0.329 e. $\AA^{-3}$ 


\section{6. ${ }^{1} \mathrm{H}$ and ${ }^{13} \mathrm{C}$ NMR spectra}

${ }^{1} \mathrm{H}$ NMR spectrum $(400 \mathrm{MHz})$ of product $\mathbf{1 b}\left(\mathrm{CDCl}_{3}\right)$

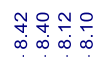

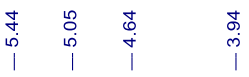

Ns $\mathrm{NOBoc}$

1b

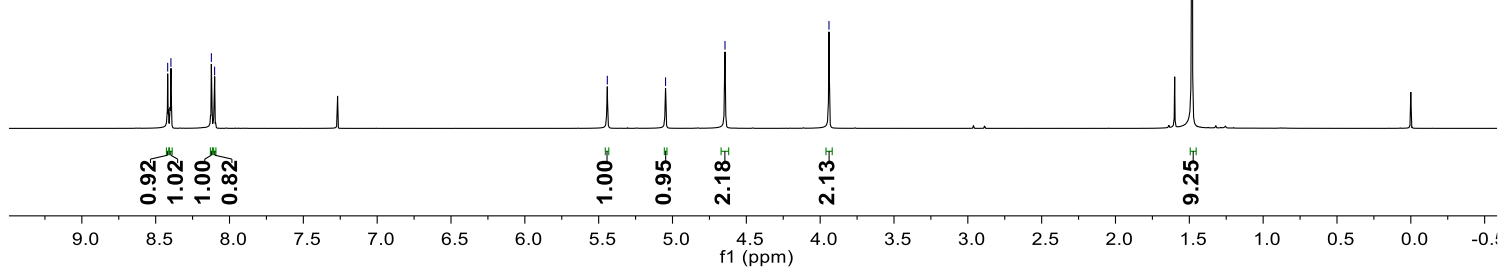

${ }^{13} \mathrm{C}$ NMR spectrum $(100 \mathrm{MHz})$ of product $\mathbf{1 b}\left(\mathrm{CDCl}_{3}\right)$<smiles>C=C(C[NH3+])CO[R16](=O)OCc1ccccc1</smiles>

$1 b$

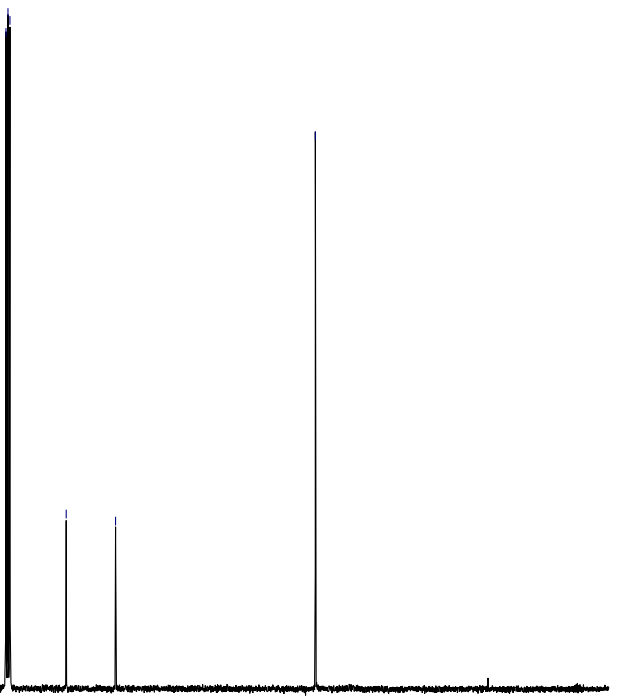


${ }^{1} \mathrm{H}$ NMR spectrum $(400 \mathrm{MHz})$ of product $3 \mathbf{b a}\left(\mathrm{CDCl}_{3}\right)$

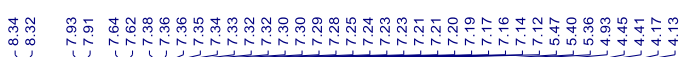

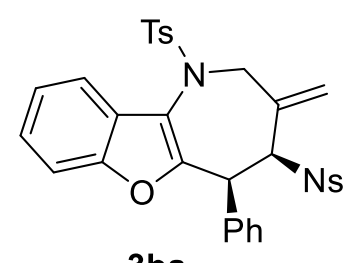

$3 \mathrm{ba}$

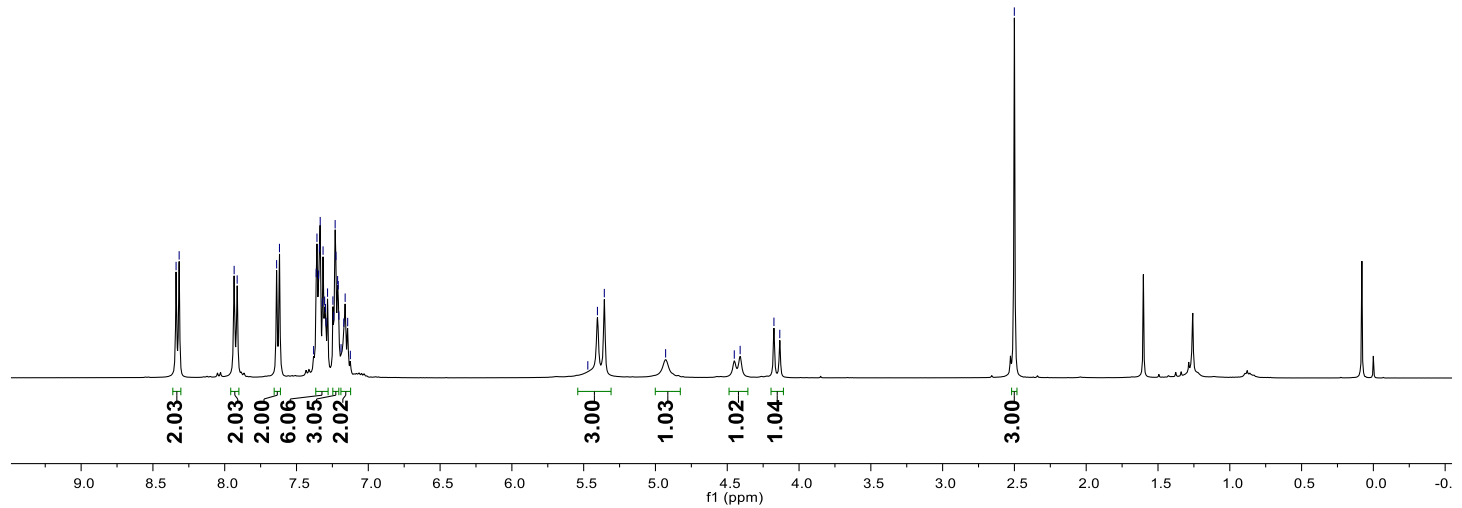

${ }^{13} \mathrm{C}$ NMR spectrum $(100 \mathrm{MHz})$ of product $3 \mathbf{b a}\left(\mathrm{CDCl}_{3}\right)$

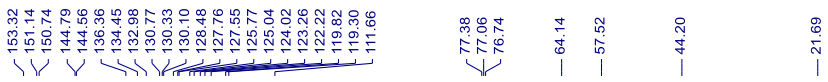

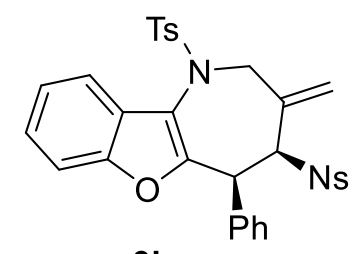

$3 \mathrm{ba}$

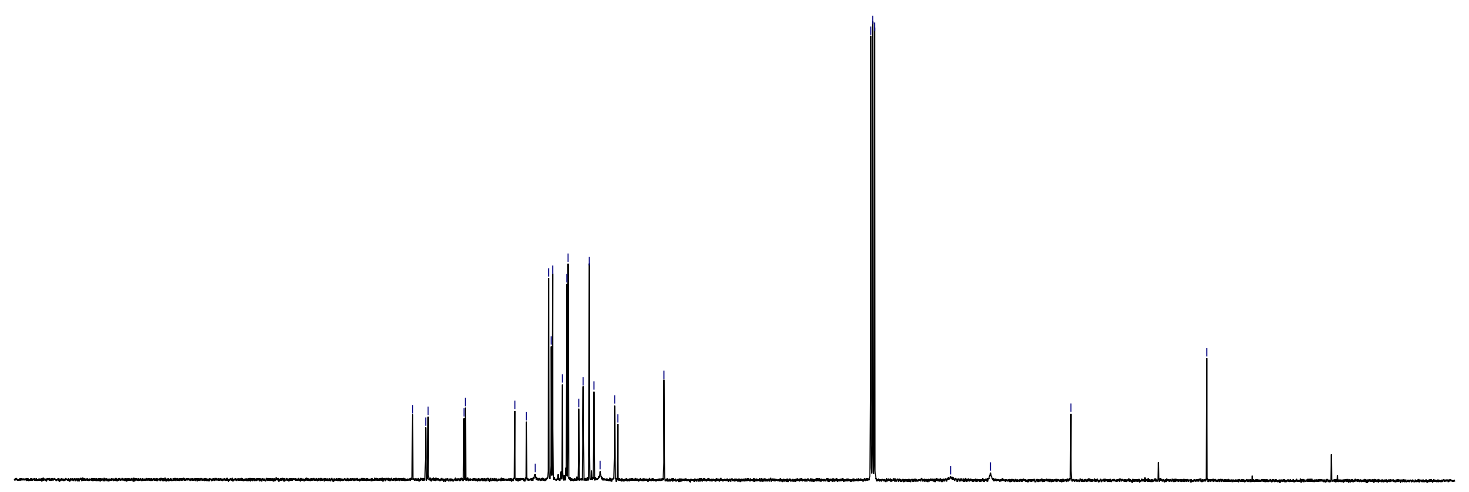

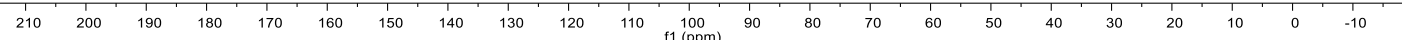


${ }^{1} \mathrm{H}$ NMR spectrum $(400 \mathrm{MHz})$ of product $3 \mathbf{b b}\left(\mathrm{CDCl}_{3}\right)$

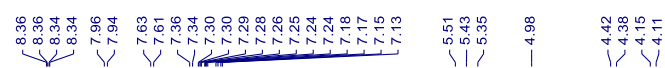<smiles>C=C1CN([13CH3])c2c(oc3ccccc23)[C@@H](c2ccc(Cl)cc2)[C@@H]1N</smiles>

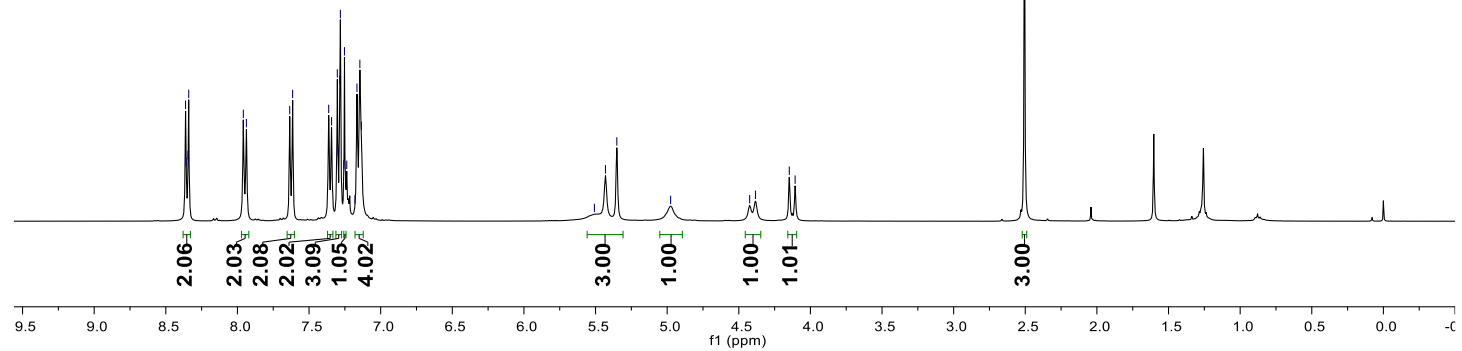

${ }^{13} \mathrm{C}$ NMR spectrum $(100 \mathrm{MHz})$ of product $3 \mathbf{b b}\left(\mathrm{CDCl}_{3}\right)$

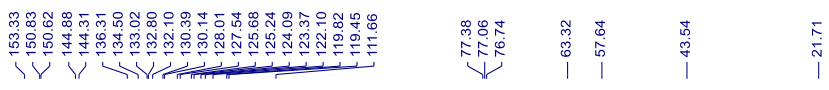<smiles>C=C1CN([As])c2c(oc3ccccc23)[C@@H](c2ccc(Cl)cc2)[C@@H]1N</smiles>

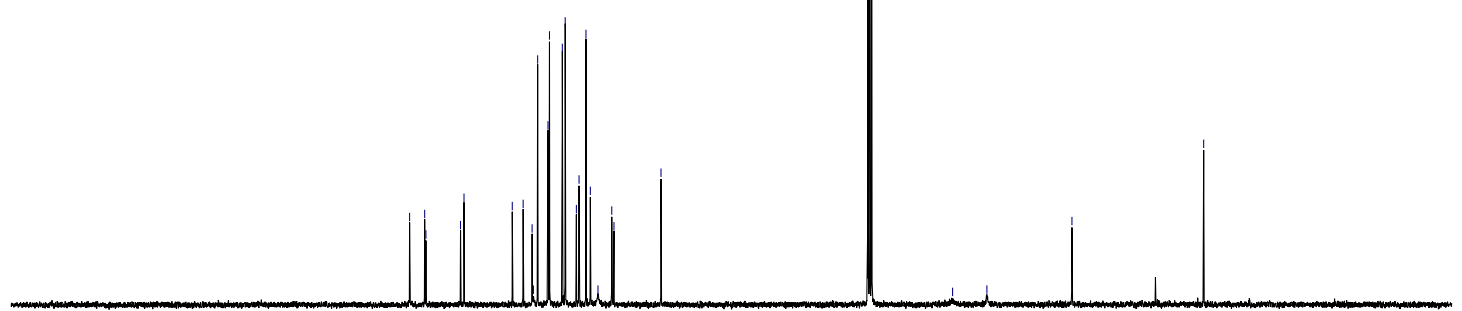

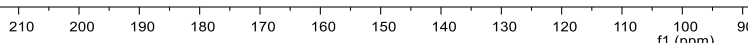


${ }^{1} \mathrm{H}$ NMR spectrum $(400 \mathrm{MHz})$ of product $3 \mathbf{b c}\left(\mathrm{CDCl}_{3}\right)$

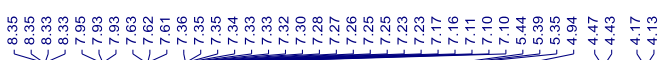
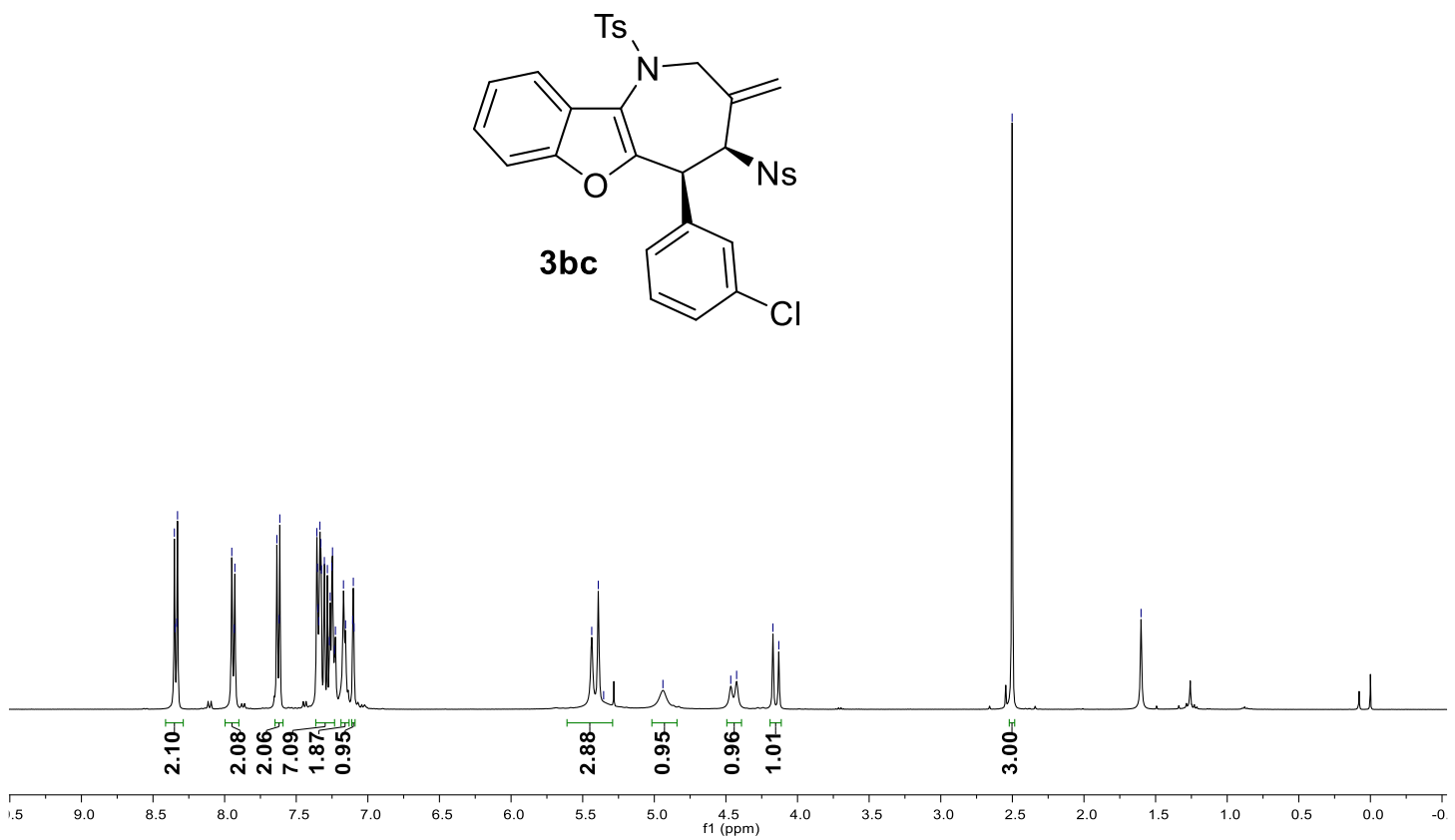

${ }^{13} \mathrm{C}$ NMR spectrum $(100 \mathrm{MHz})$ of product $3 \mathrm{bc}\left(\mathrm{CDCl}_{3}\right)$

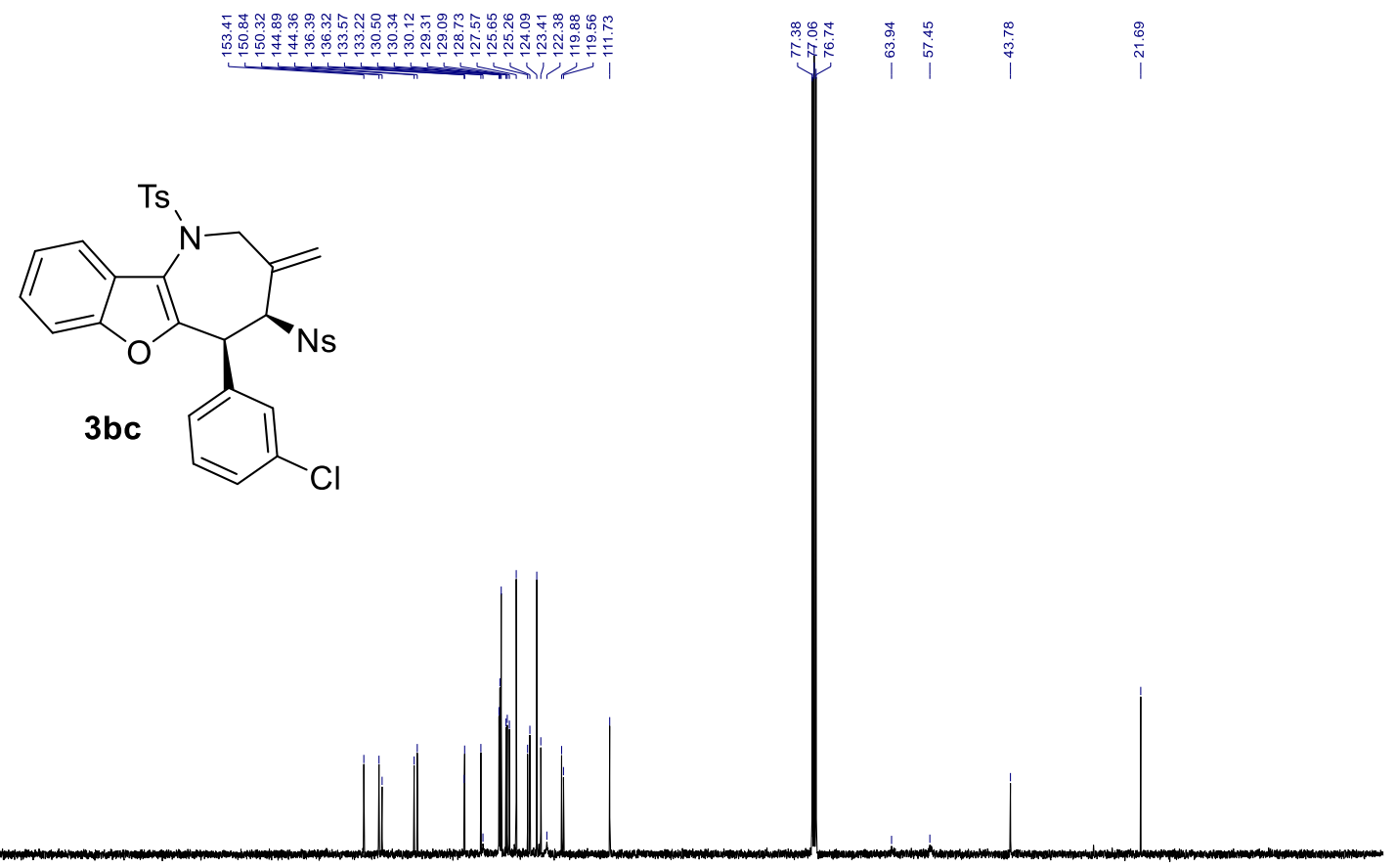

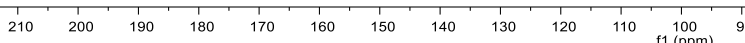


${ }^{1} \mathrm{H}$ NMR spectrum $(400 \mathrm{MHz})$ of product $3 \mathrm{bd}\left(\mathrm{CDCl}_{3}\right)(\mathrm{dr}=2: 1)$

疍

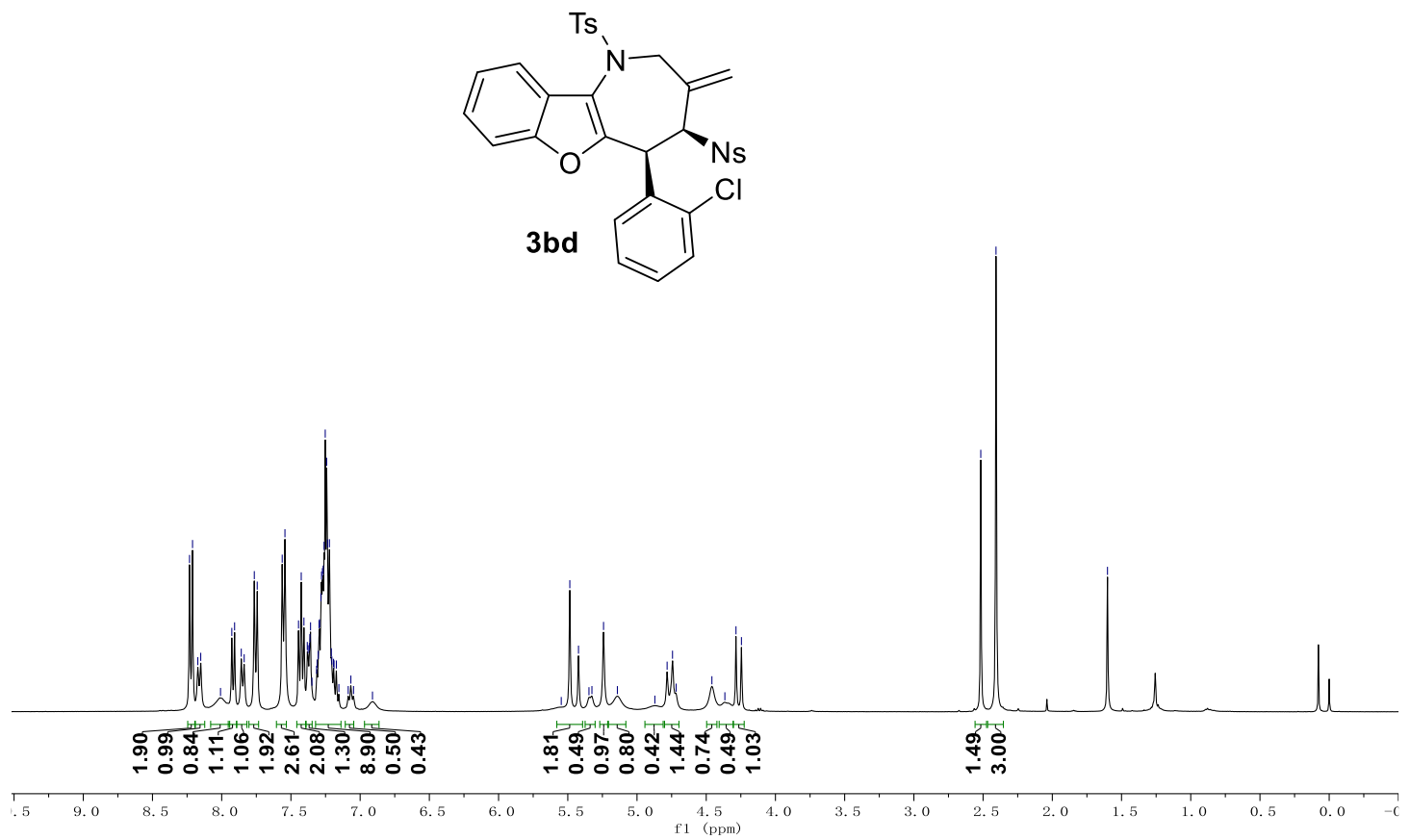

${ }^{13} \mathrm{C}$ NMR spectrum $(100 \mathrm{MHz})$ of product $3 \mathbf{b d}\left(\mathrm{CDCl}_{3}\right)$

|<smiles>C=C1CN([125I])c2c(oc3ccccc23)C(c2ccccc2Cl)C1N</smiles>

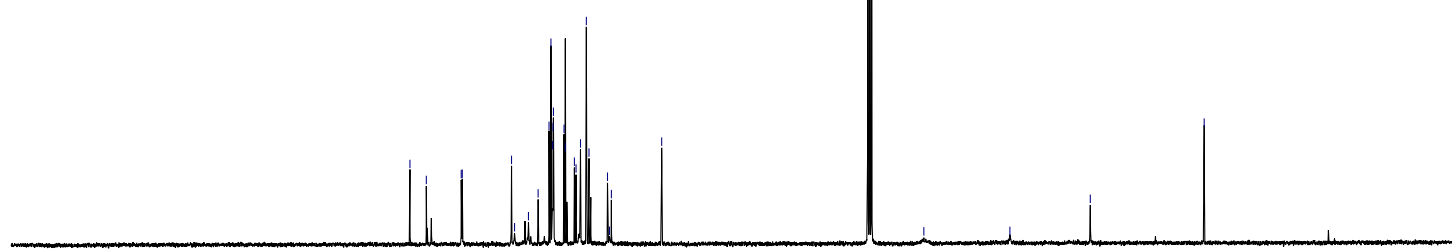

$\begin{array}{lllllllllllll}210 & 200 & 190 & 180 & 170 & 160 & 150 & 140 & 130 & 120 & 110 & 100 & 9 \\ & & & & & & & & 100 & (\mathrm{pmm}) & \end{array}$ 
${ }^{1} \mathrm{H}$ NMR spectrum $(400 \mathrm{MHz})$ of product $3 \mathrm{be}\left(\mathrm{CDCl}_{3}\right)$

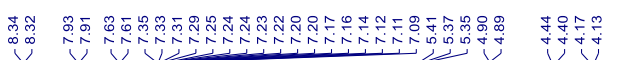

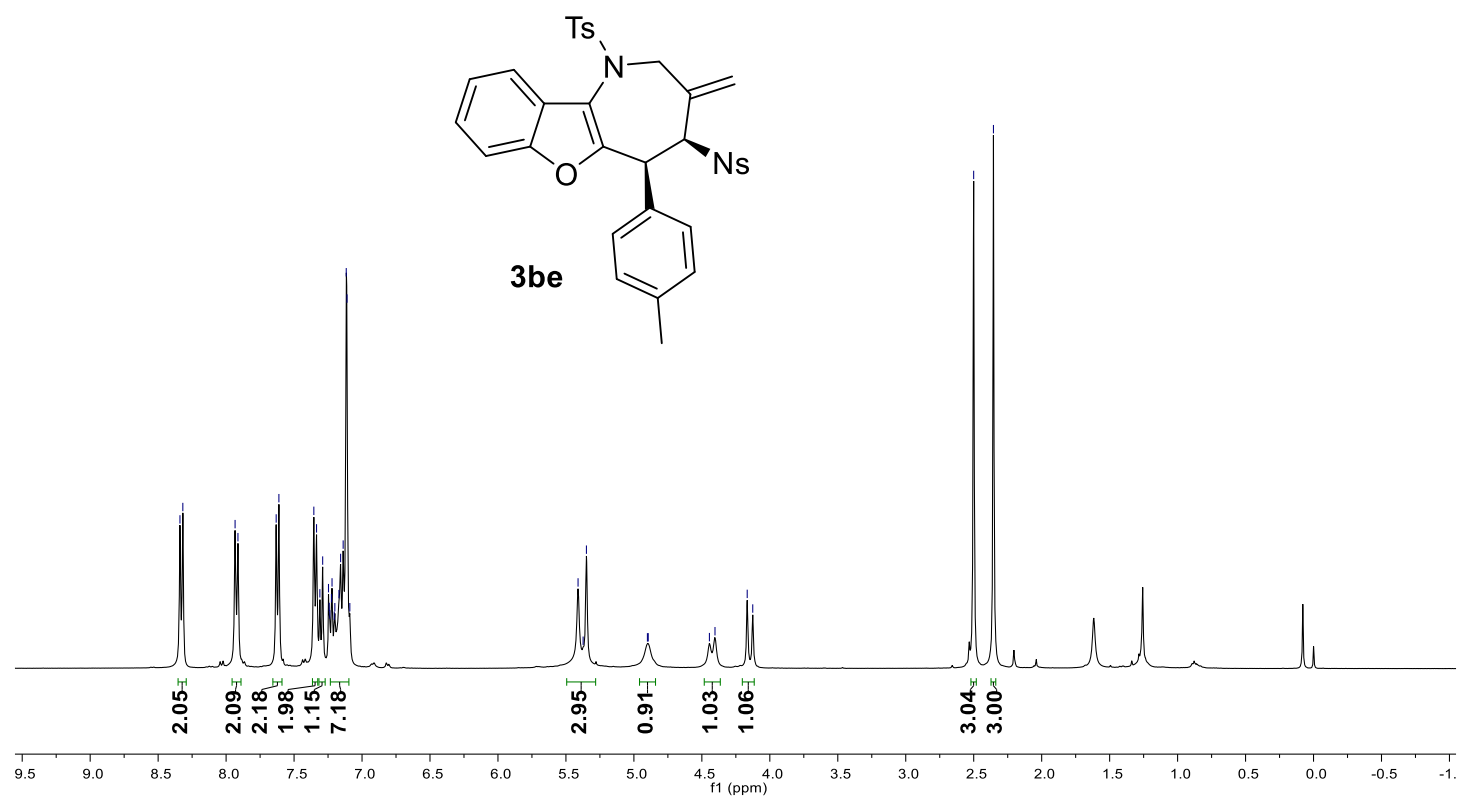

${ }^{13} \mathrm{C}$ NMR spectrum $(100 \mathrm{MHz})$ of product $3 \mathrm{be}\left(\mathrm{CDCl}_{3}\right)$

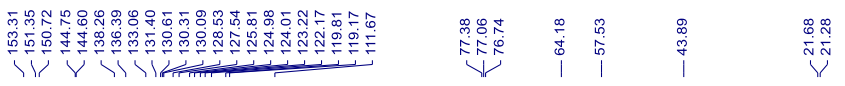<smiles>C=C1CN([12F])c2c(oc3ccccc23)C(c2ccc(C)cc2)C1N</smiles>

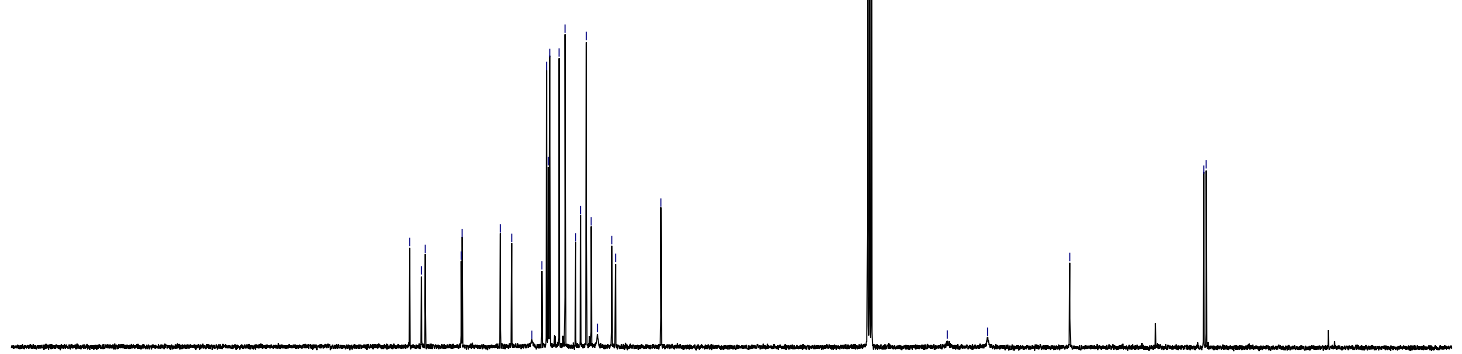

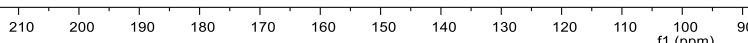


${ }^{1} \mathrm{H}$ NMR spectrum $(400 \mathrm{MHz})$ of product $3 \mathbf{b f}\left(\mathrm{CDCl}_{3}\right)$

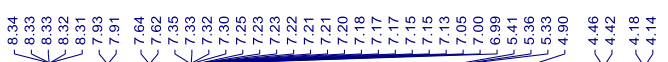<smiles>[B]N1CC(=C)[C@H](N)[C@H](c2cccc(C)c2)c2oc3ccccc3c21</smiles>

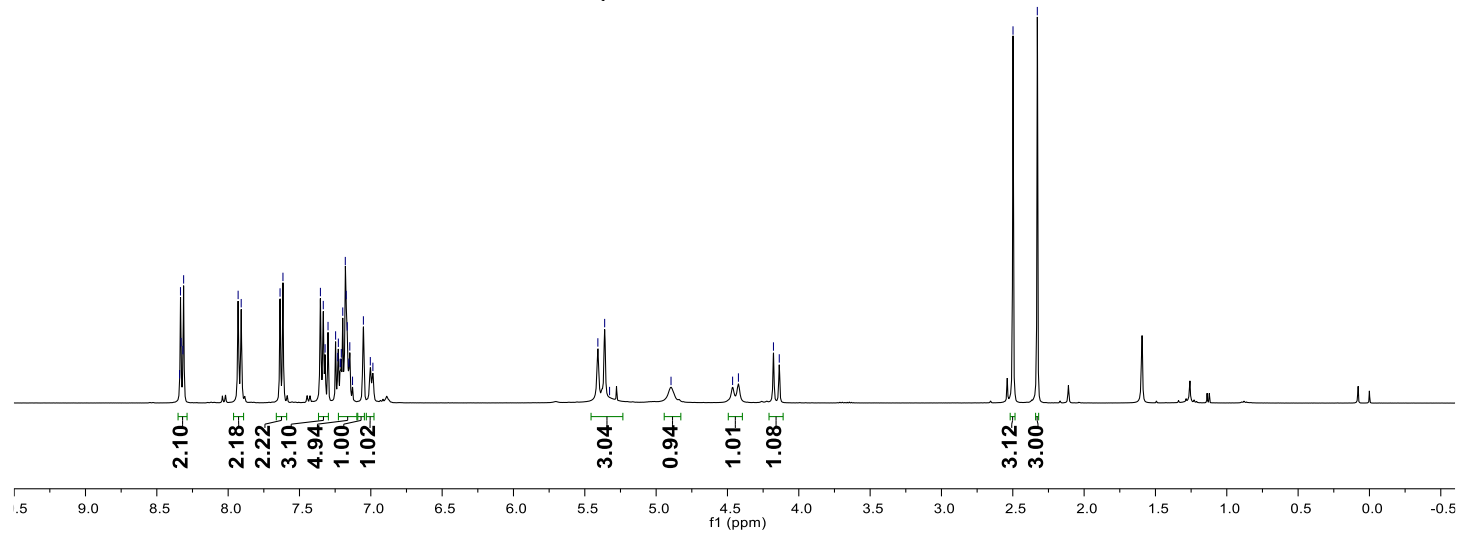

${ }^{13} \mathrm{C}$ NMR spectrum $(100 \mathrm{MHz})$ of product $\mathbf{3 b f}\left(\mathrm{CDCl}_{3}\right)$

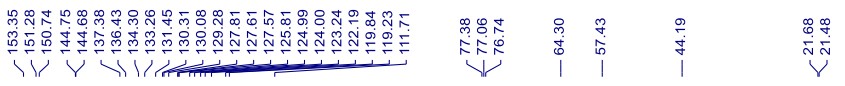<smiles>C=C1CN([As])c2c(oc3ccccc23)[C@@H](c2cccc(C)c2)[C@@H]1N</smiles>

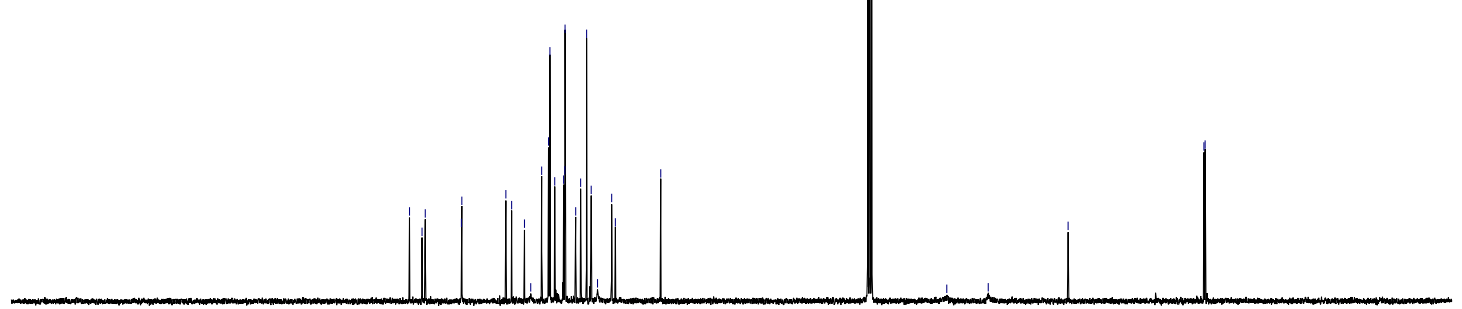

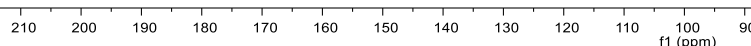


${ }^{1} \mathrm{H}$ NMR spectrum $(400 \mathrm{MHz})$ of product $3 \mathrm{bg}\left(\mathrm{CDCl}_{3}\right)$

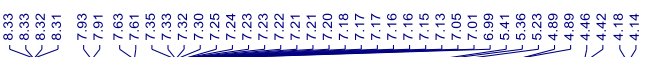<smiles>C=C1CN([Tl])c2c(oc3ccccc23)[C@@H](c2ccccc2C)[C@@H]1N</smiles>

${ }^{13} \mathrm{C}$ NMR spectrum $(100 \mathrm{MHz})$ of product $3 \mathrm{bg}\left(\mathrm{CDCl}_{3}\right)$

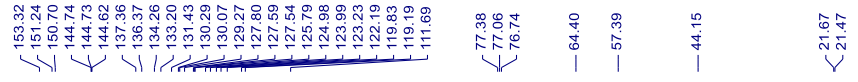<smiles>C=C1CN([12F])c2c(oc3ccccc23)[C@@H](c2ccccc2C)[C@@H]1N</smiles>

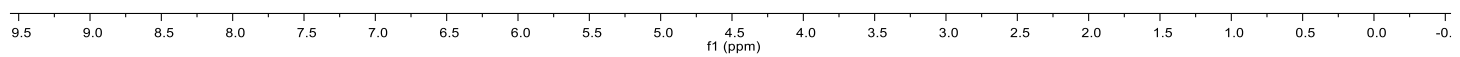

(3) $N$ MR spectum (100

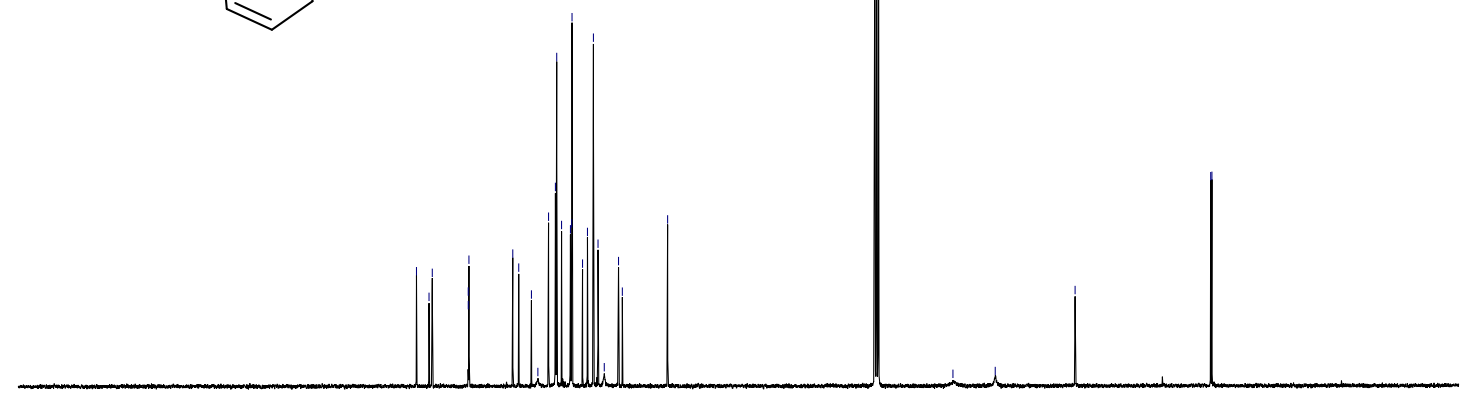


${ }^{1} \mathrm{H}$ NMR spectrum $(400 \mathrm{MHz})$ of product $3 \mathbf{b h}\left(\mathrm{CDCl}_{3}\right)$

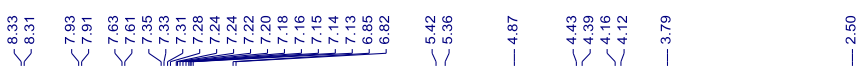
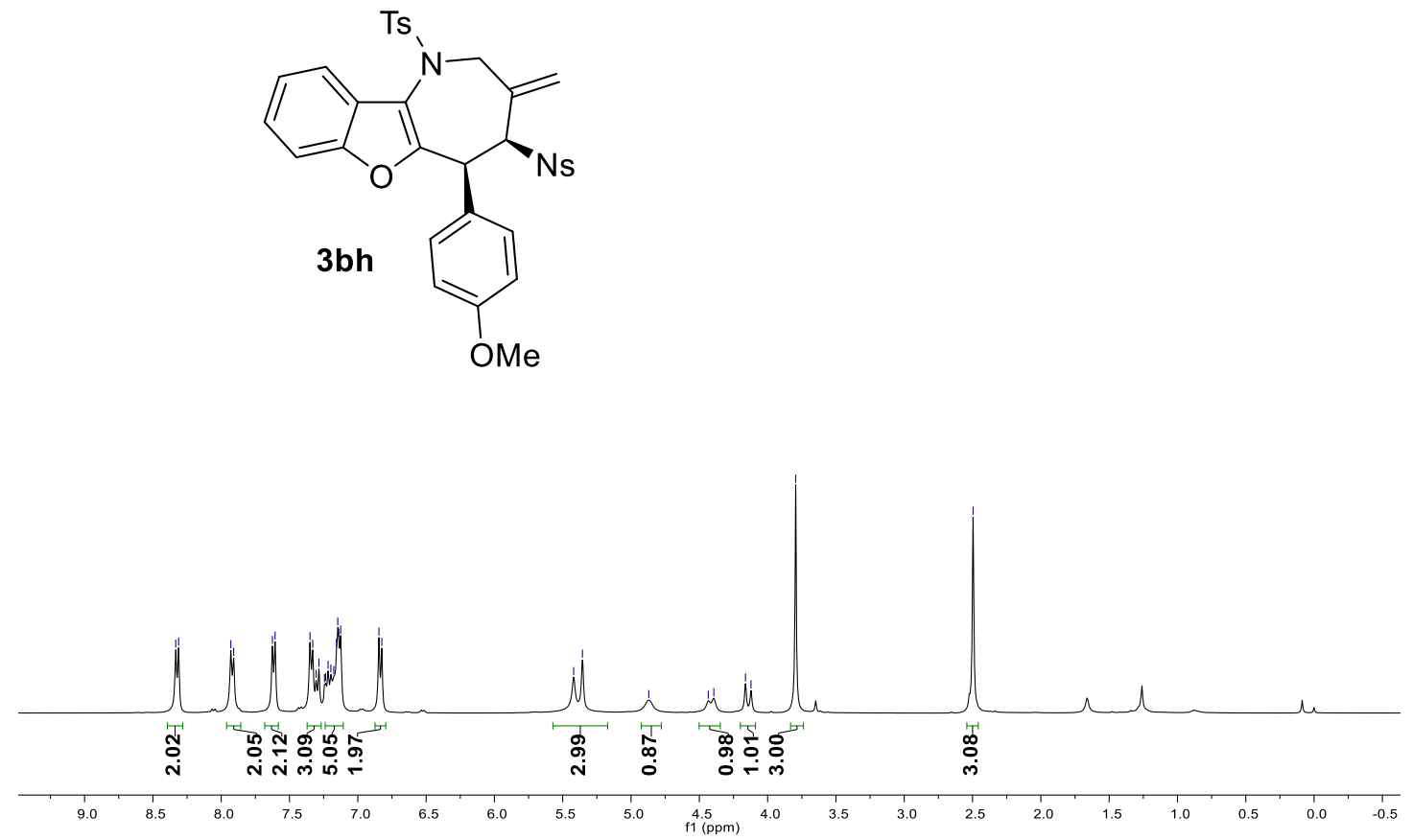

${ }^{13} \mathrm{C}$ NMR spectrum $(100 \mathrm{MHz})$ of product $3 \mathrm{bh}\left(\mathrm{CDCl}_{3}\right)$

|

Ts<smiles>C=C1CN(C)c2c(oc3ccccc23)[C@@H](c2ccc(OC)cc2)C1[N+](=O)[O-]</smiles>

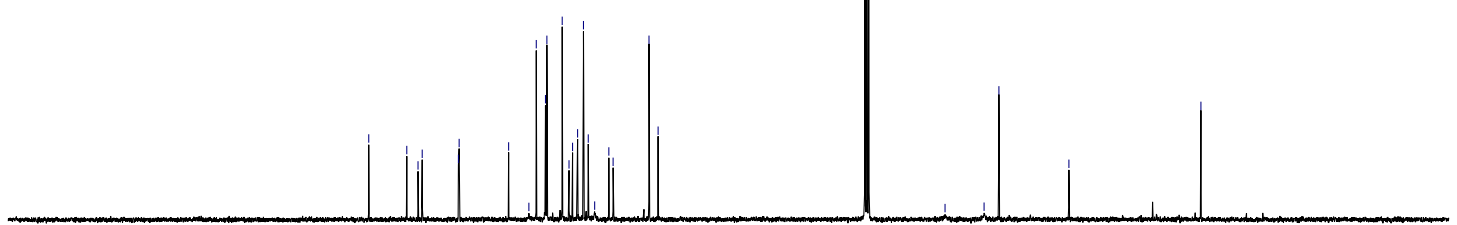

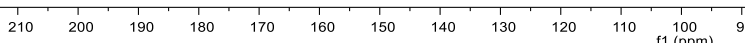


${ }^{1} \mathrm{H}$ NMR spectrum $(400 \mathrm{MHz})$ of product $3 \mathbf{b i}\left(\mathrm{CDCl}_{3}\right)$
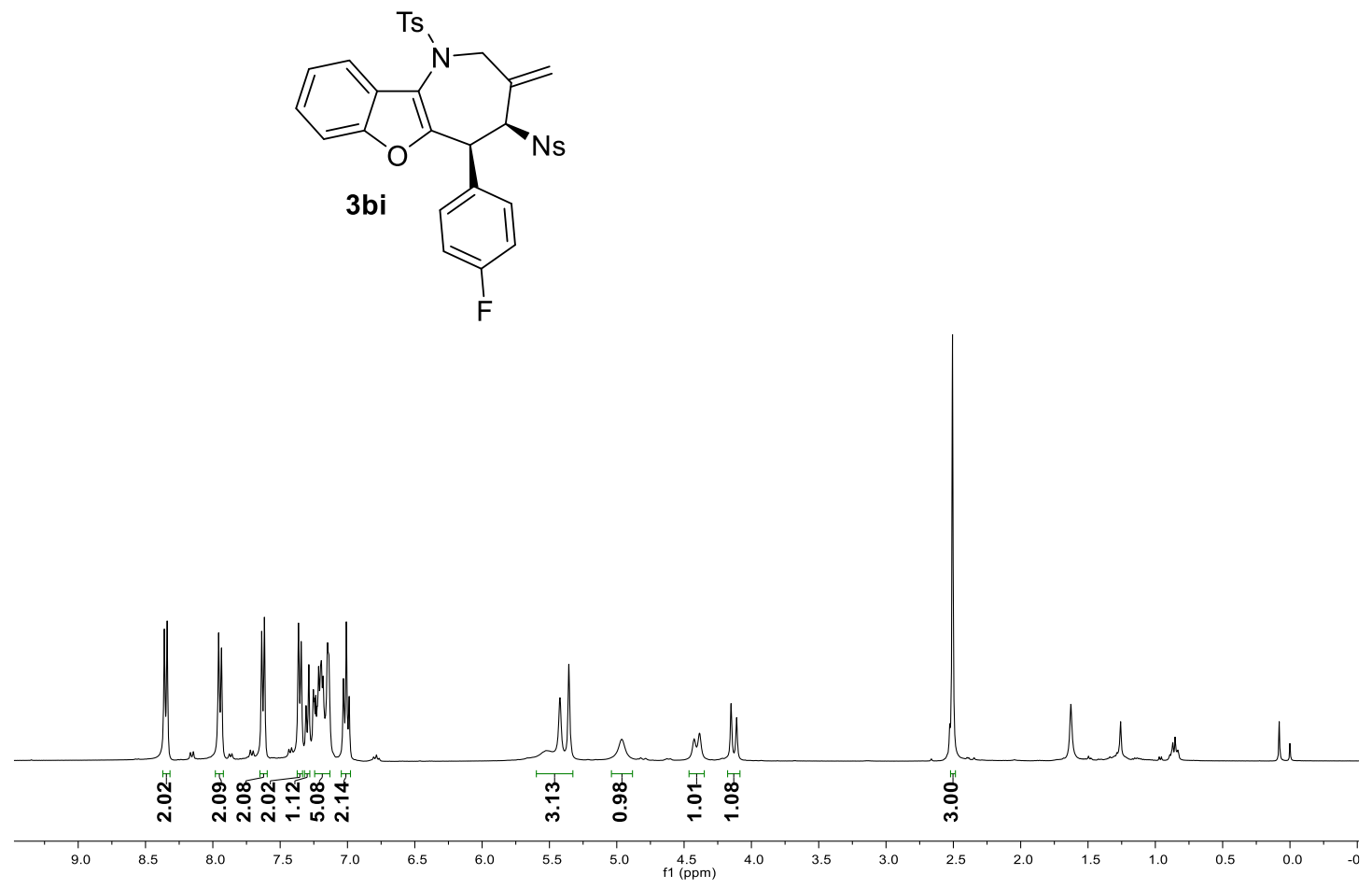

${ }^{13} \mathrm{C}$ NMR spectrum $(100 \mathrm{MHz})$ of product $3 \mathbf{b i}\left(\mathrm{CDCl}_{3}\right)$

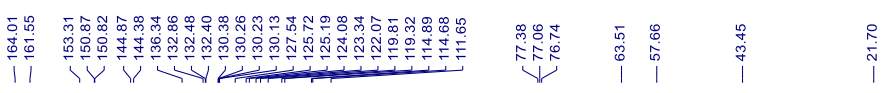<smiles>C=C1CN([125I])c2c(oc3ccccc23)C(c2ccc(F)cc2)C1[N+](=O)[O-]</smiles>

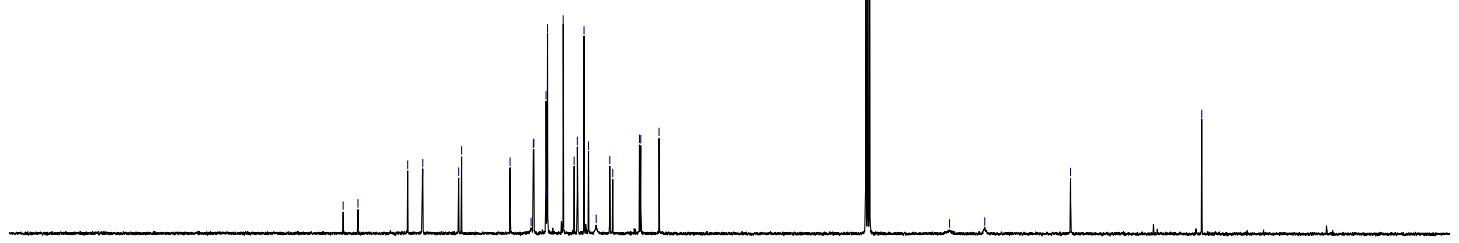

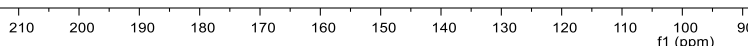


${ }^{1} \mathrm{H}$ NMR spectrum $(400 \mathrm{MHz})$ of product $\mathbf{3 b j}\left(\mathrm{CDCl}_{3}\right)$

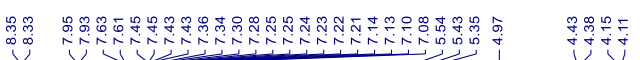
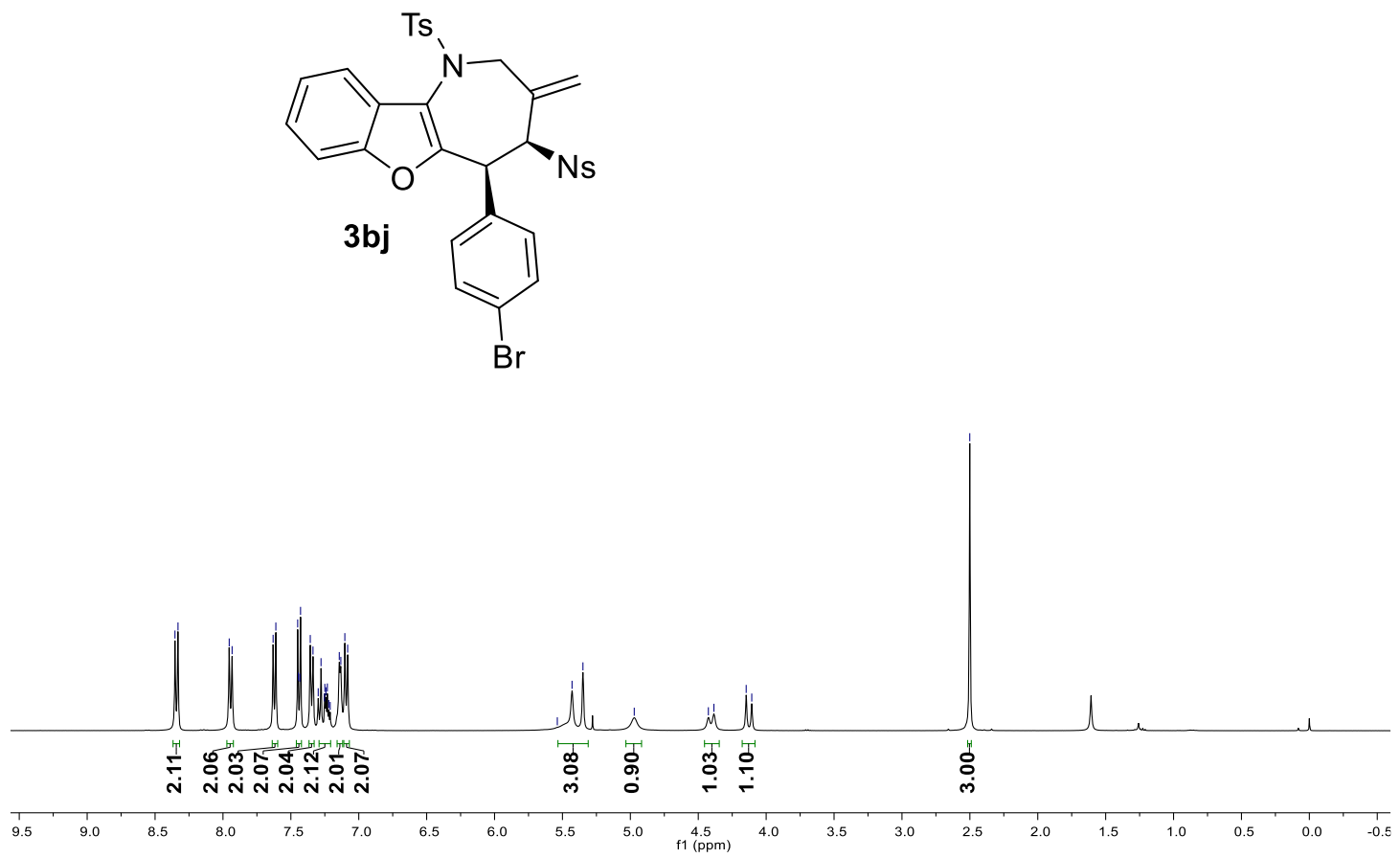

${ }^{13} \mathrm{C}$ NMR spectrum (100 MHz) of product $3 \mathbf{b j}\left(\mathrm{CDCl}_{3}\right)$

$\underbrace{V}$<smiles>C=C1CN([As])c2c(oc3ccccc23)[C@H](c2ccc(Br)cc2)[C@H]1N</smiles>

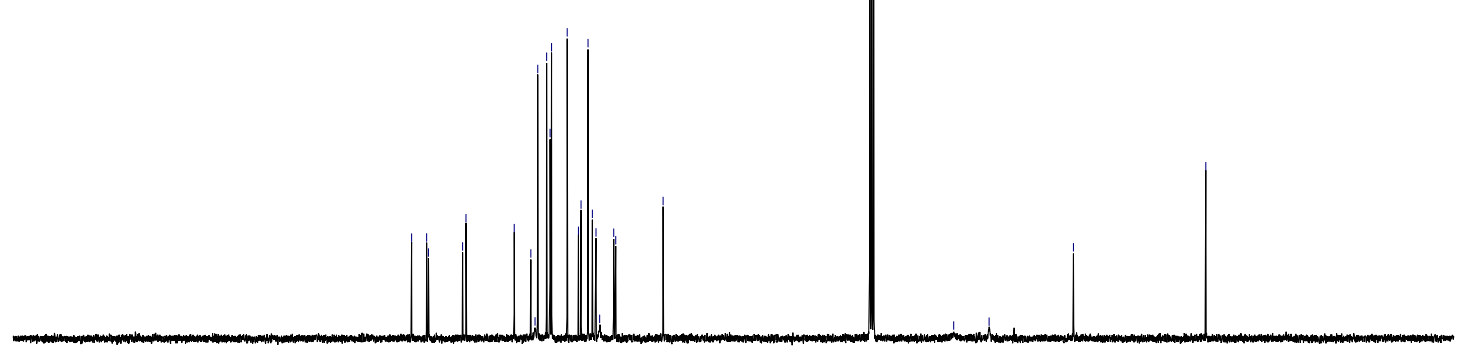

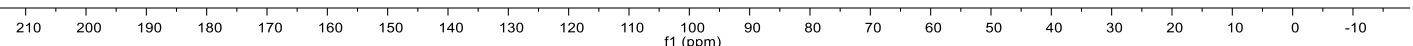


${ }^{1} \mathrm{H}$ NMR spectrum $(400 \mathrm{MHz})$ of product $3 \mathbf{b k}\left(\mathrm{CDCl}_{3}\right)$

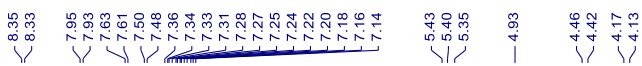
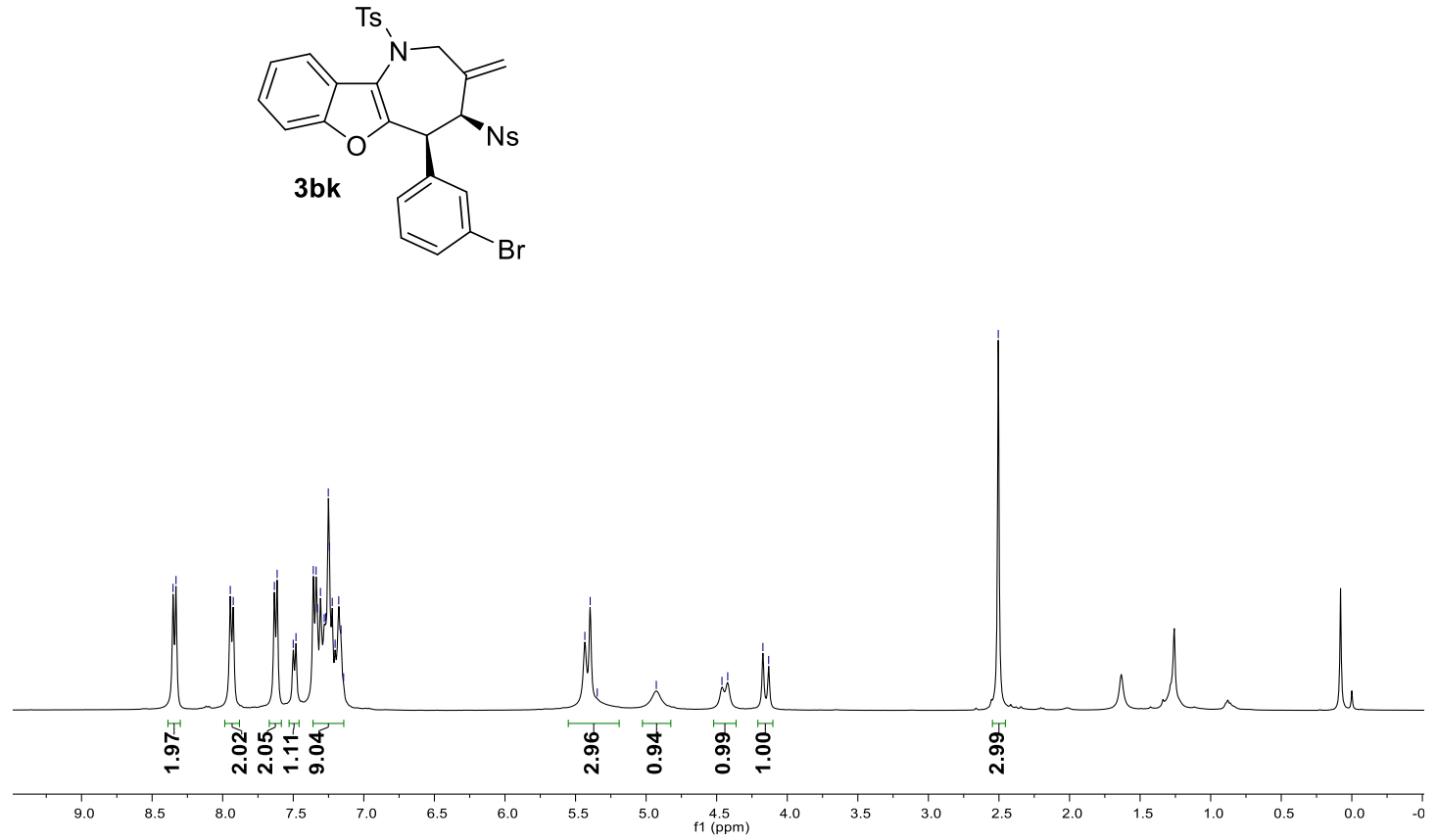

${ }^{13} \mathrm{C}$ NMR spectrum $(100 \mathrm{MHz})$ of product $3 \mathbf{b k}\left(\mathrm{CDCl}_{3}\right)$

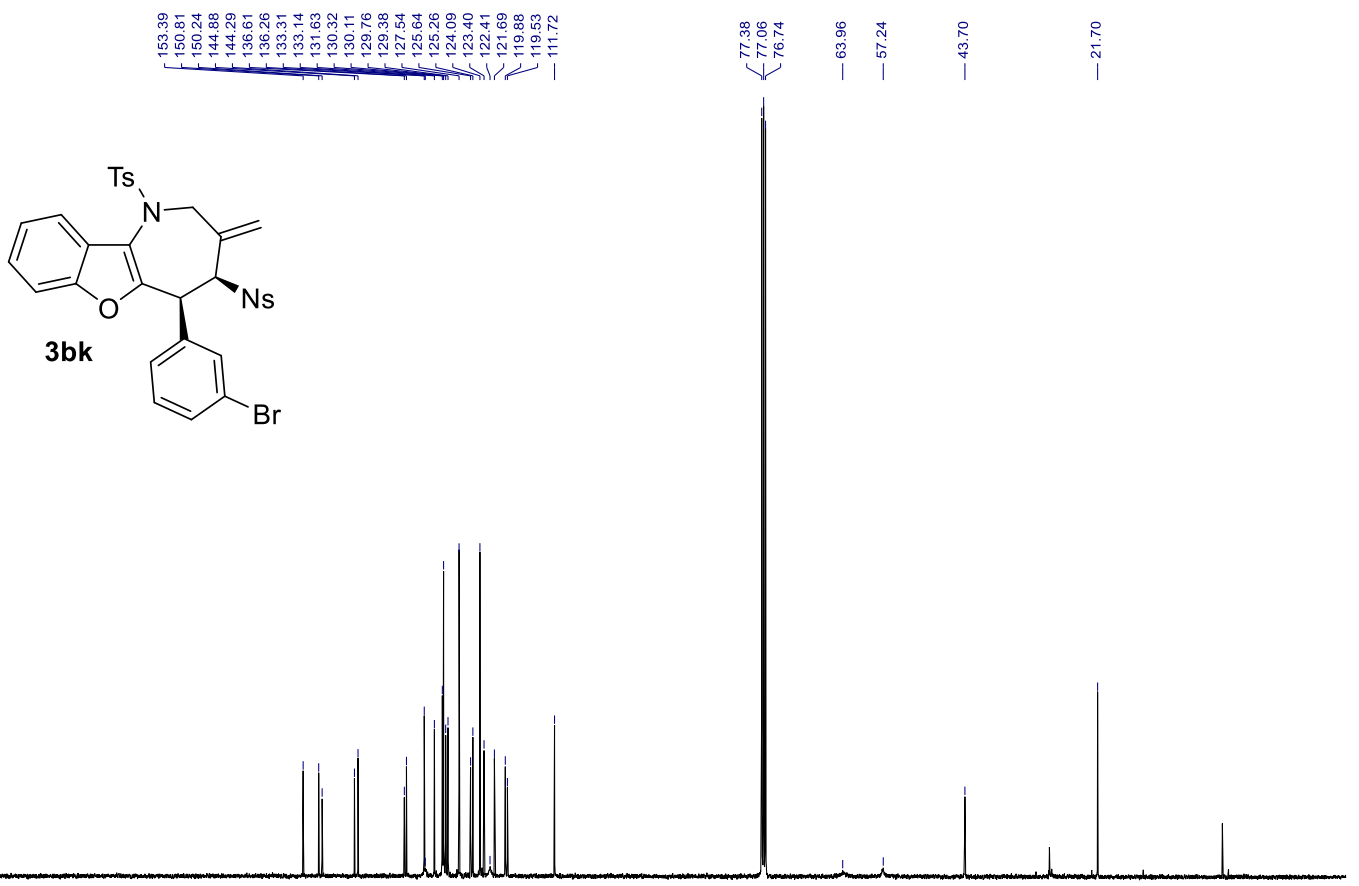

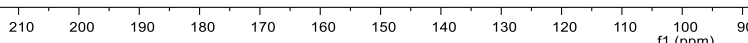


${ }^{1} \mathrm{H}$ NMR spectrum $(400 \mathrm{MHz})$ of product $3 \mathbf{b l}\left(\mathrm{CDCl}_{3}\right)$

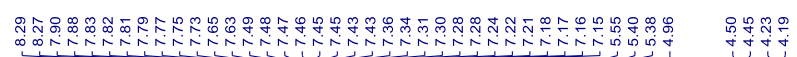

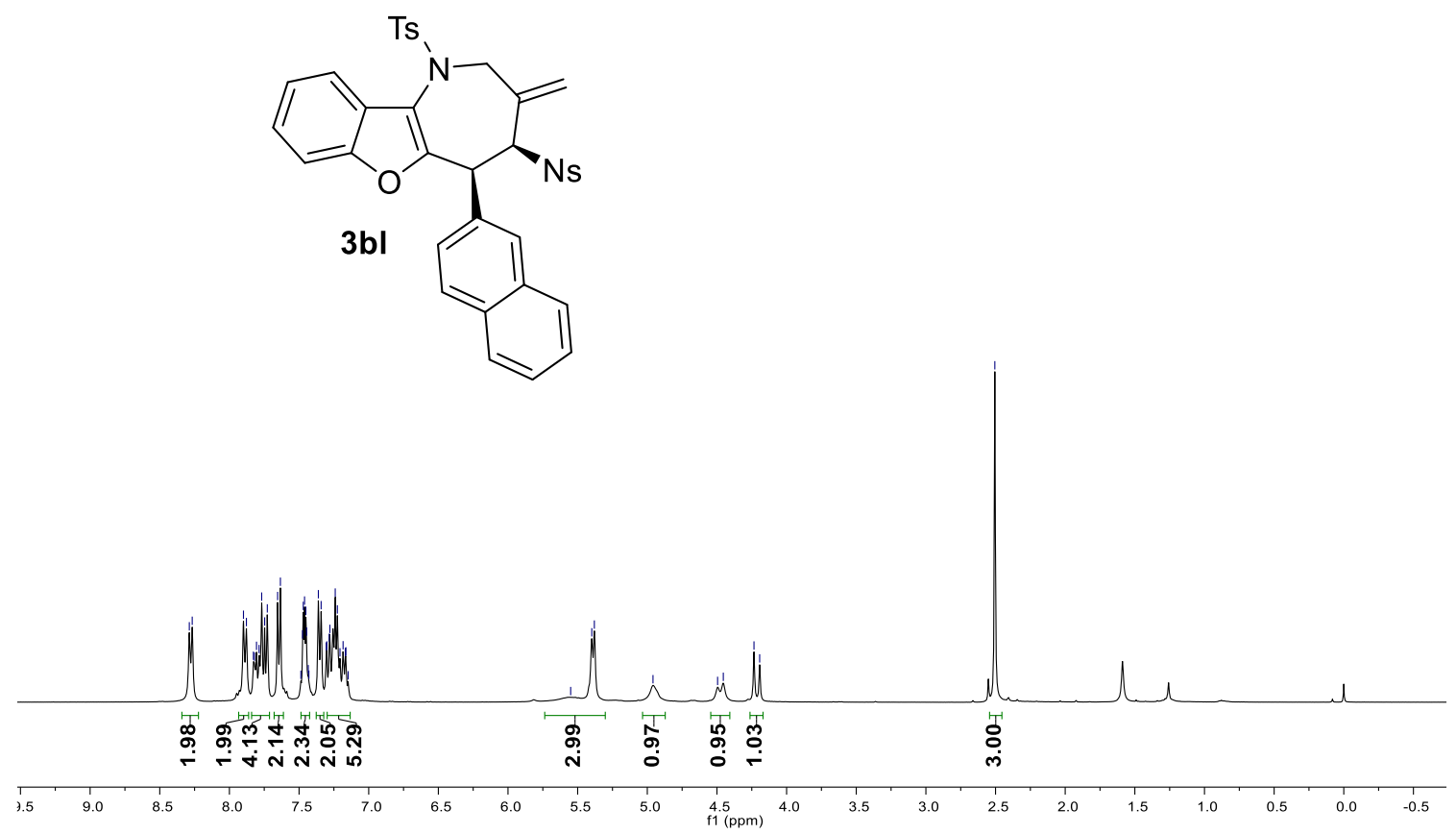

${ }^{13} \mathrm{C}$ NMR spectrum $(100 \mathrm{MHz})$ of product $3 \mathrm{bl}\left(\mathrm{CDCl}_{3}\right)$

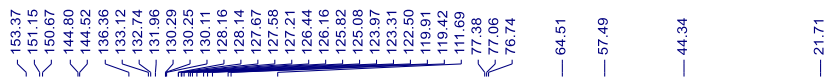<smiles>C=C1CN([As])c2c(oc3ccccc23)[C@@H]1c1ccc2ccccc2c1</smiles>

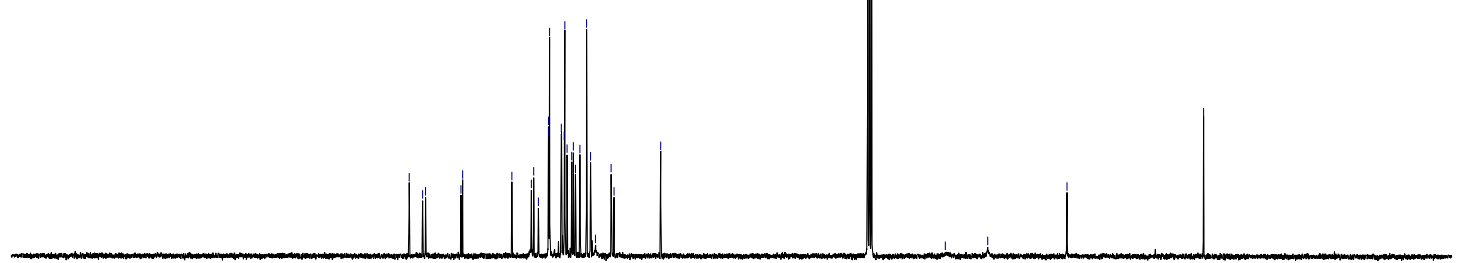

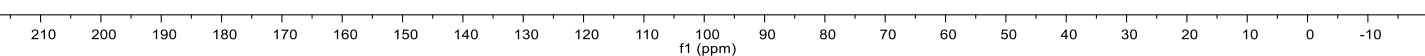


${ }^{1} \mathrm{H}$ NMR spectrum $(400 \mathrm{MHz})$ of product $3 \mathrm{bm}\left(\mathrm{CDCl}_{3}\right)$

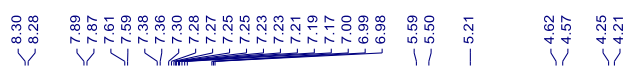
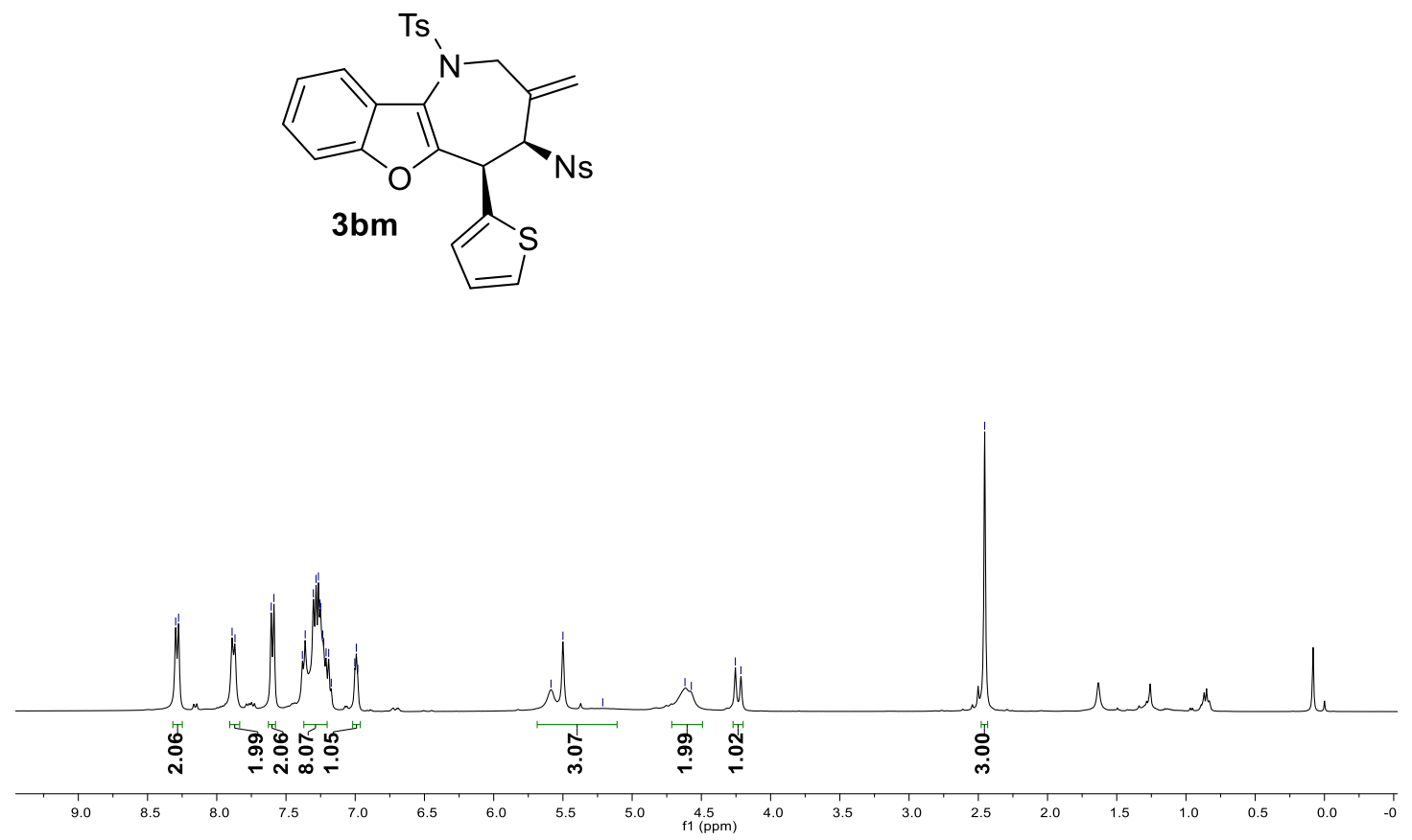

${ }^{13} \mathrm{C}$ NMR spectrum $(100 \mathrm{MHz})$ of product $3 \mathrm{bm}\left(\mathrm{CDCl}_{3}\right)$

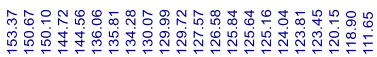

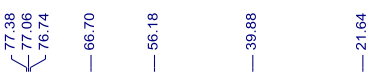<smiles>C=C1CN([As])c2c(oc3ccccc23)[C@H](c2cccs2)[C@@H]1N</smiles>

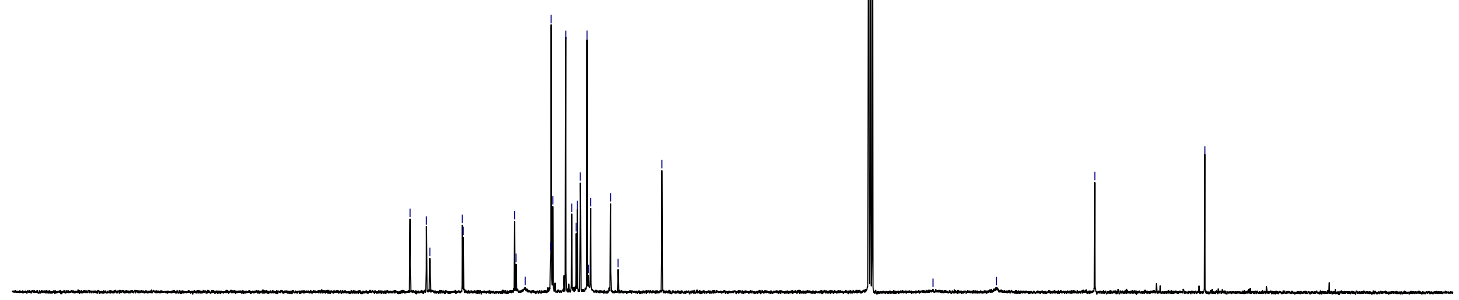

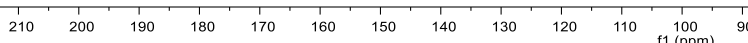


${ }^{1} \mathrm{H}$ NMR spectrum $(400 \mathrm{MHz})$ of product $3 \mathbf{b n}\left(\mathrm{CDCl}_{3}\right)$

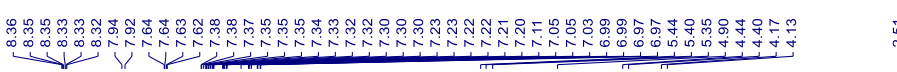<smiles>C=C1CN([As])c2c(oc3cc(C)ccc23)[C@@H](c2ccccc2)[C@H]1N</smiles>

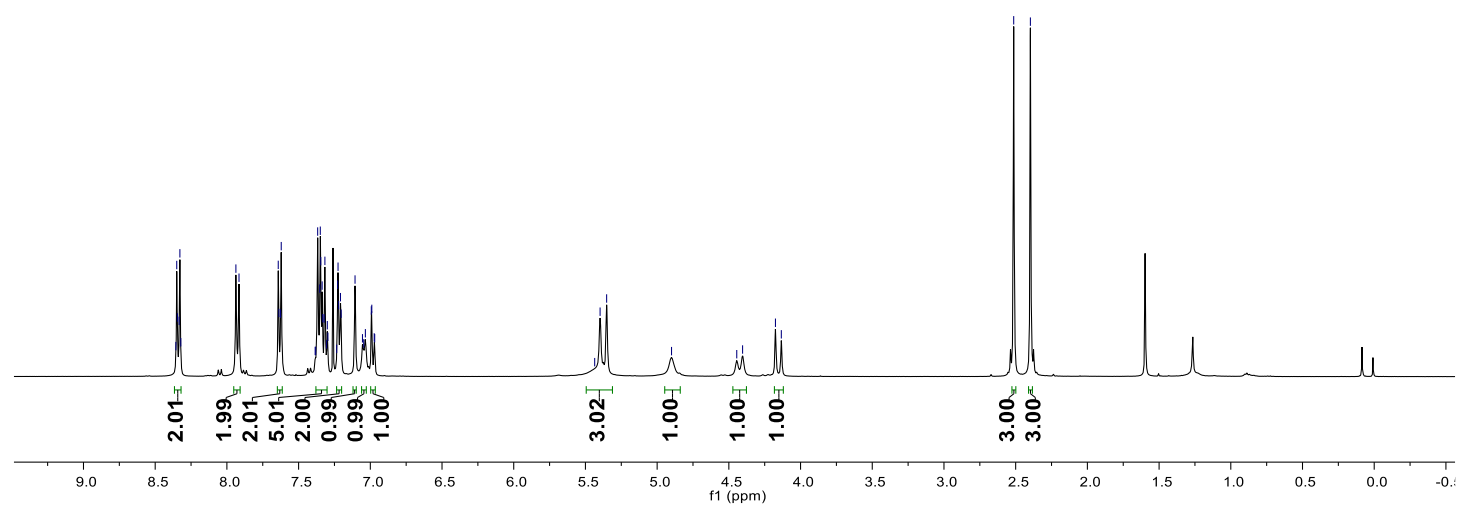

${ }^{13} \mathrm{C}$ NMR spectrum $(100 \mathrm{MHz})$ of product $3 \mathrm{bn}\left(\mathrm{CDCl}_{3}\right)$

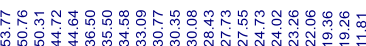

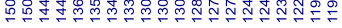

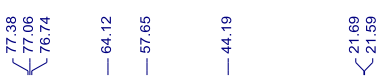
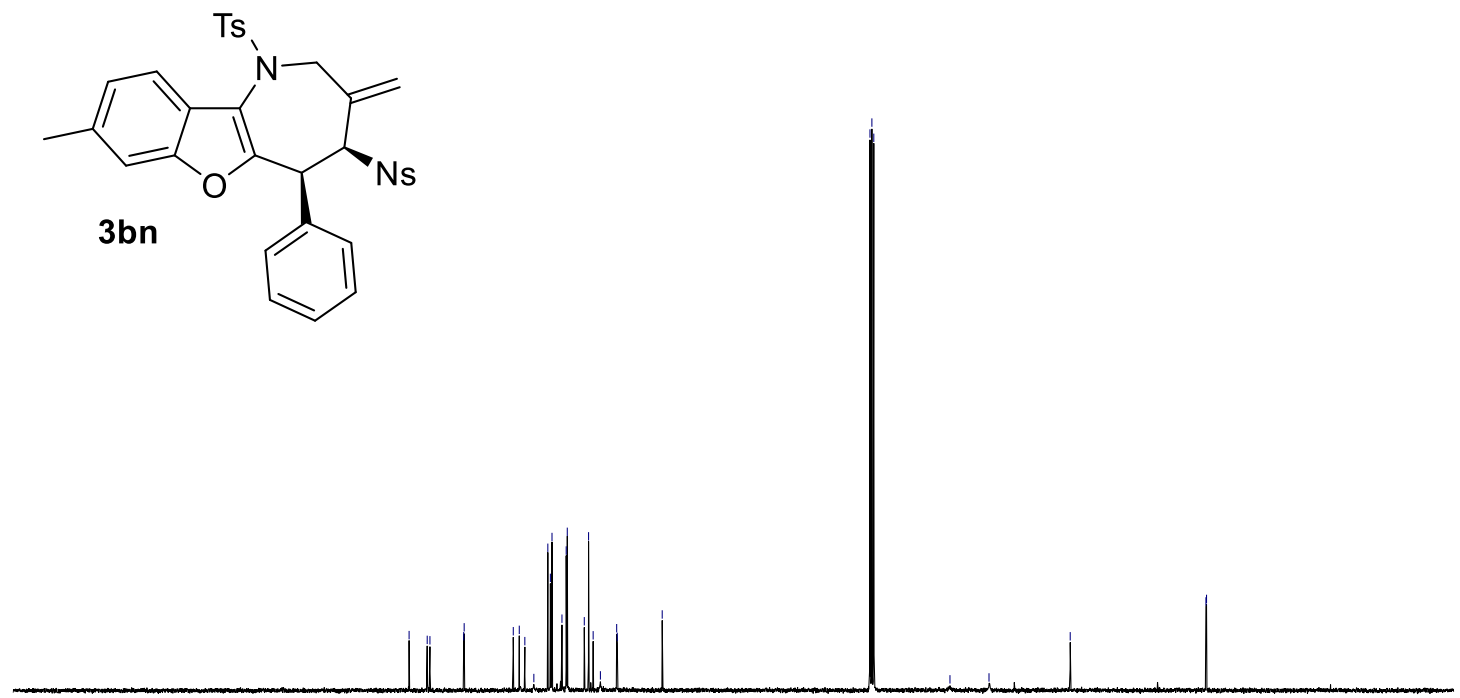
${ }^{1} \mathrm{H}$ NMR spectrum $(400 \mathrm{MHz})$ of product $3 \mathbf{b o}\left(\mathrm{CDCl}_{3}\right)$

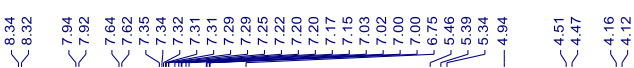<smiles>C=C1CN([13CH3])c2c(oc3ccc(C)cc23)[C@@H](c2ccccc2)[C@H]1N</smiles>

$\downarrow$

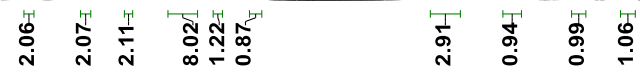

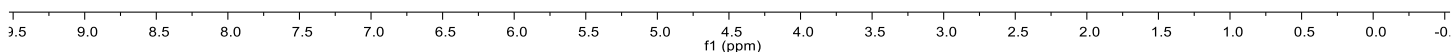

${ }^{13} \mathrm{C}$ NMR spectrum $(100 \mathrm{MHz})$ of product $3 \mathrm{bo}\left(\mathrm{CDCl}_{3}\right)$

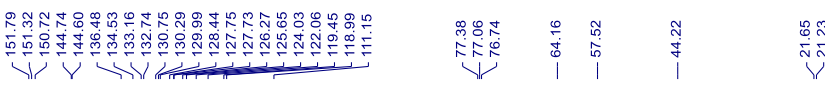<smiles>C=C1CN([As])c2c(oc3ccc(C)cc23)[C@@H](c2ccccc2)[C@H]1N</smiles>

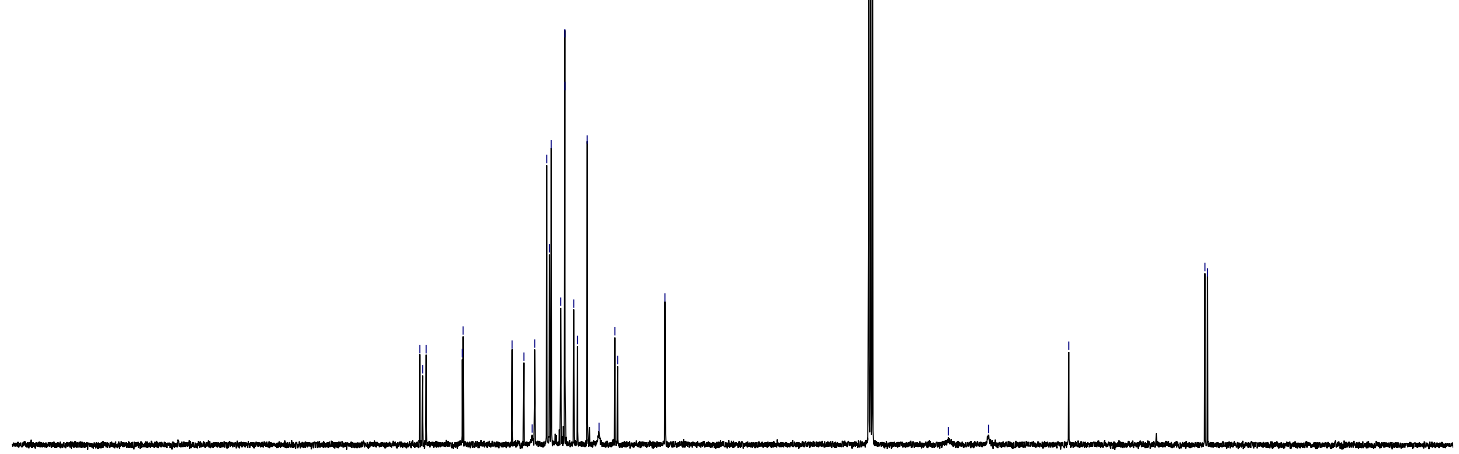


${ }^{1} \mathrm{H}$ NMR spectrum $(400 \mathrm{MHz})$ of product $3 \mathbf{b p}\left(\mathrm{CDCl}_{3}\right)$

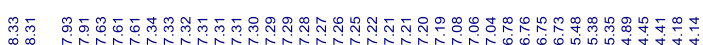

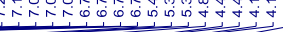
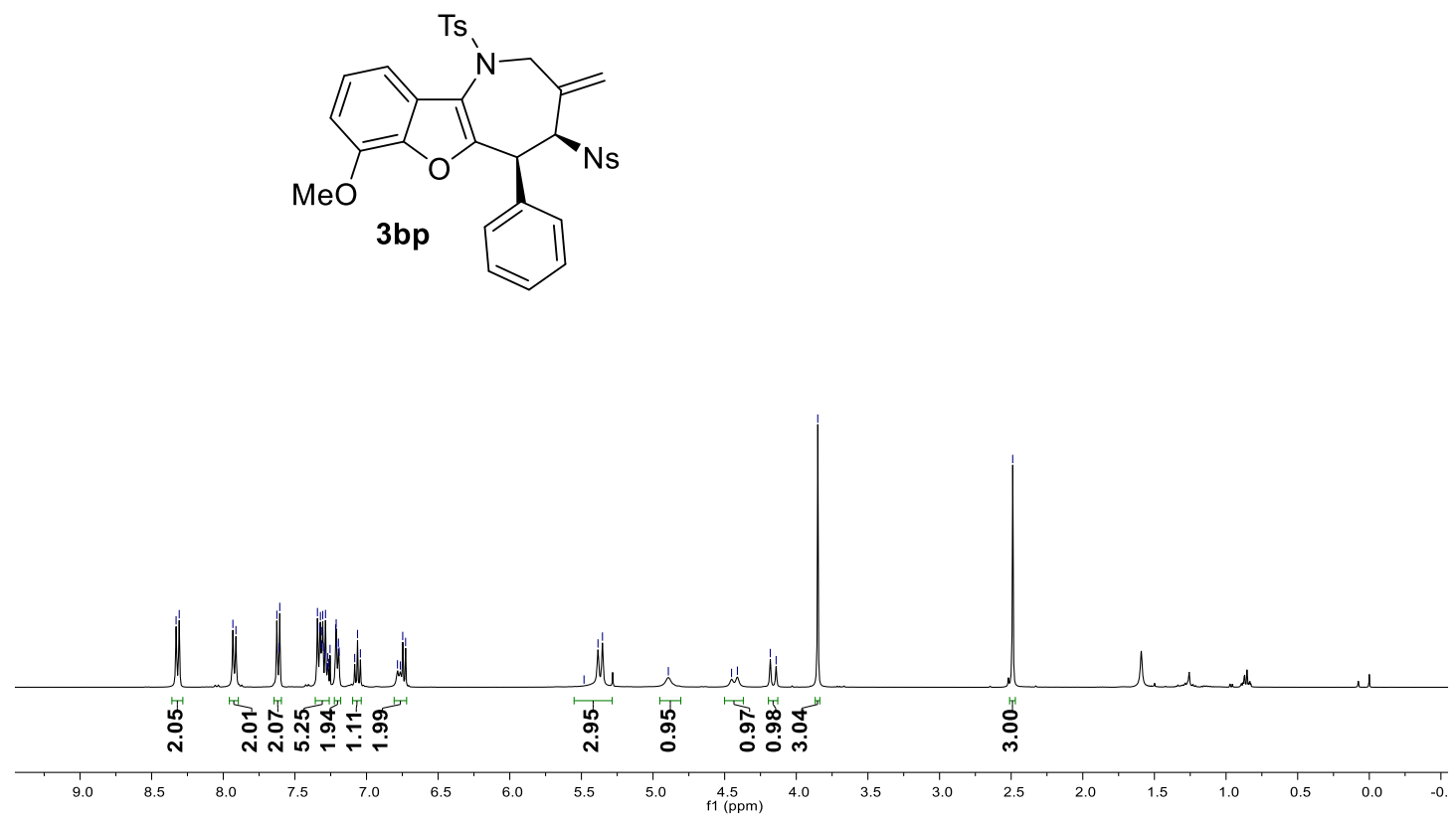

${ }^{13} \mathrm{C}$ NMR spectrum $(100 \mathrm{MHz})$ of product $3 \mathrm{bp}\left(\mathrm{CDCl}_{3}\right)$

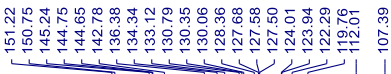<smiles>C=C1CN([12F])c2c(oc3c(OC)cccc23)C(c2ccccc2)C1[N+](=O)[O-]</smiles>
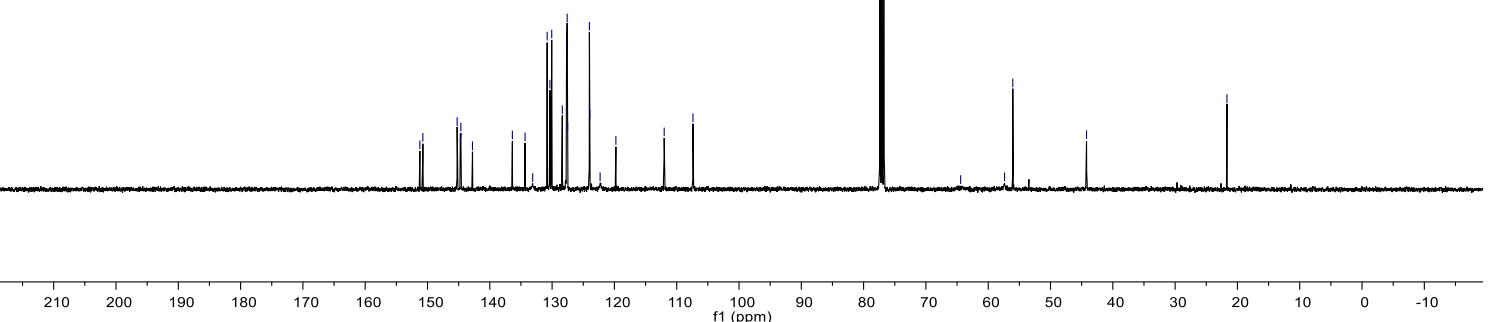
${ }^{1} \mathrm{H}$ NMR spectrum $(400 \mathrm{MHz})$ of product $\mathbf{3 b q}\left(\mathrm{CDCl}_{3}\right)$

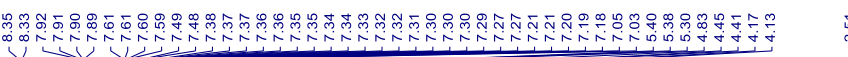<smiles>C=C1CN(C)c2c(oc3cc(Br)ccc23)C(c2ccccc2)C1N</smiles>

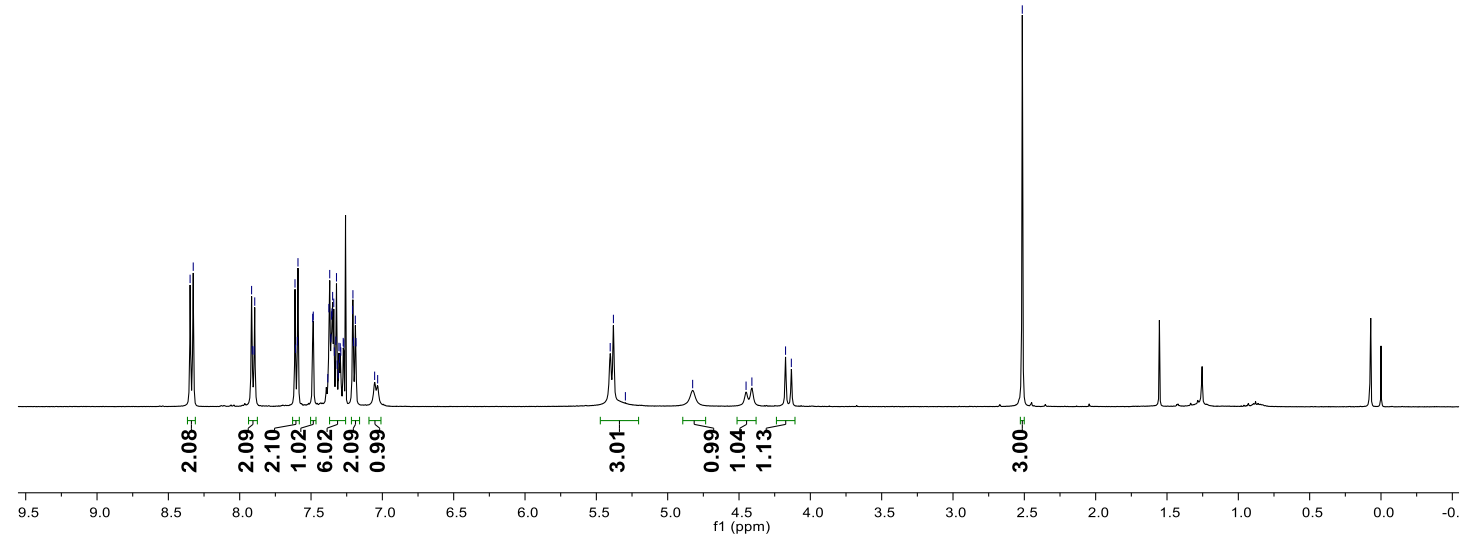

${ }^{13} \mathrm{C}$ NMR spectrum $(100 \mathrm{MHz})$ of product $3 \mathrm{bq}\left(\mathrm{CDCl}_{3}\right)$

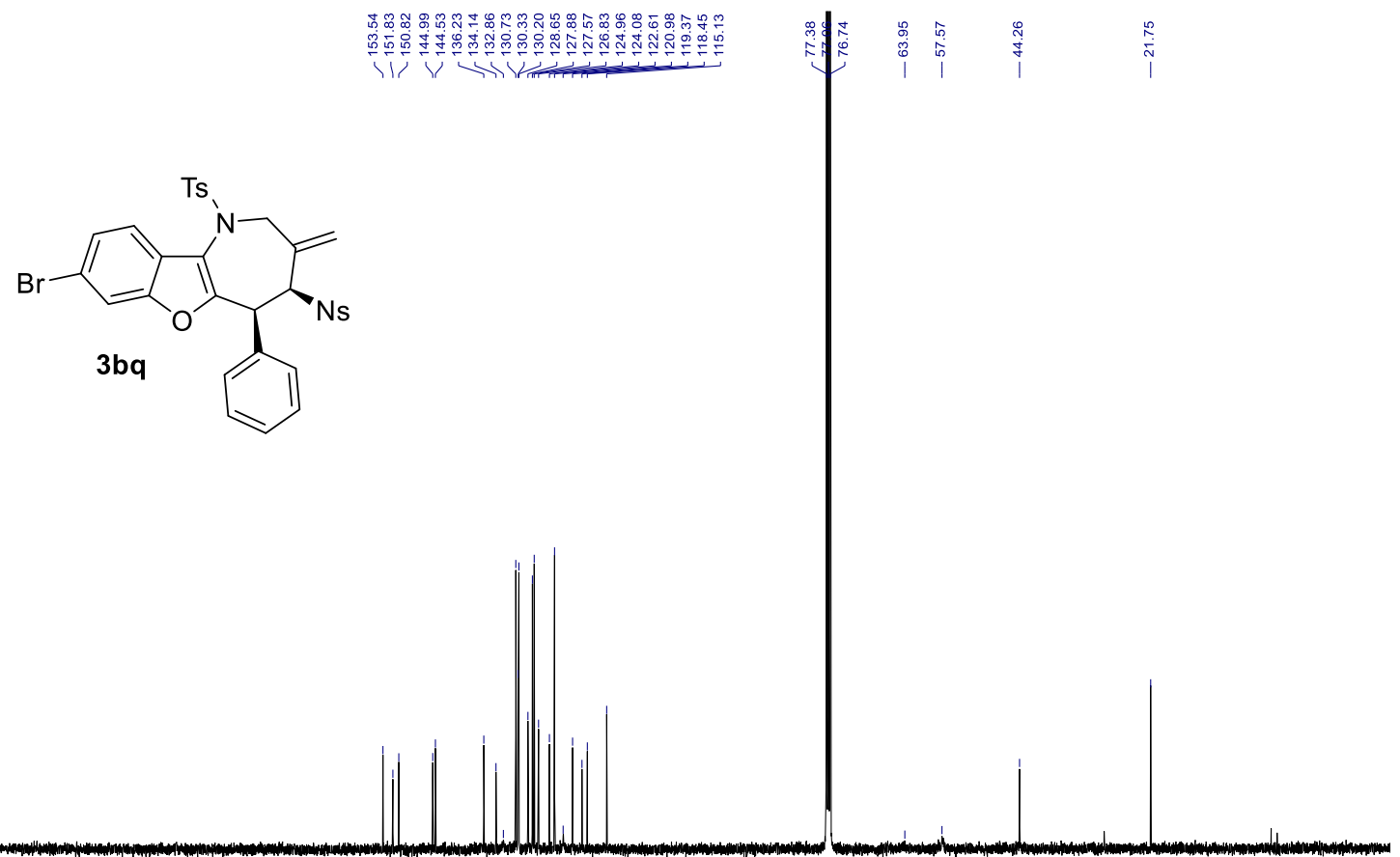

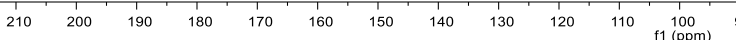


${ }^{1} \mathrm{H}$ NMR spectrum $(400 \mathrm{MHz})$ of product $3 \mathrm{br}\left(\mathrm{CDCl}_{3}\right)$

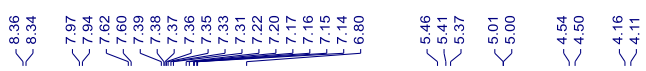
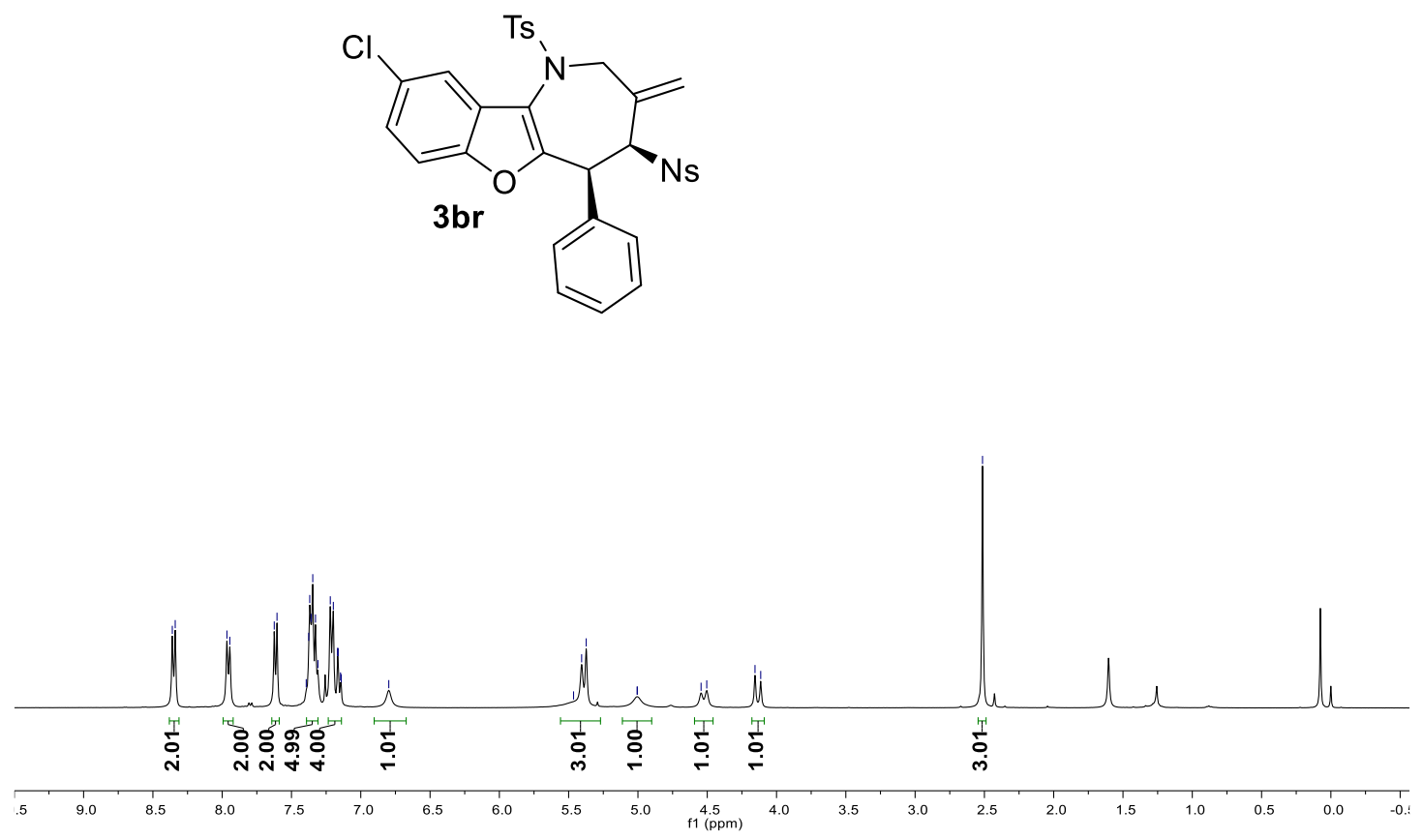

${ }^{13} \mathrm{C}$ NMR spectrum $(100 \mathrm{MHz})$ of product $3 \mathrm{br}\left(\mathrm{CDCl}_{3}\right)$

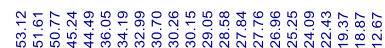

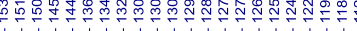

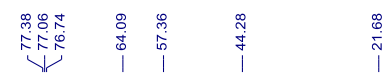<smiles>C=C1CN([As])c2c(oc3ccc(Cl)cc23)[C@@H](c2ccccc2)[C@@H]1N</smiles>

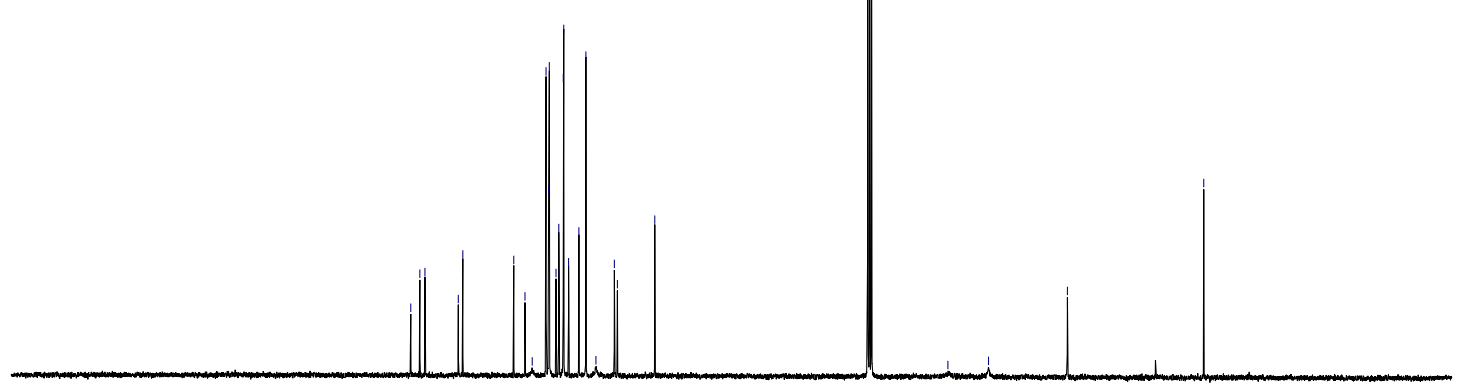

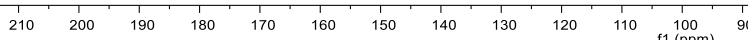


${ }^{1} \mathrm{H}$ NMR spectrum $(400 \mathrm{MHz})$ of product $3 \mathrm{bs}\left(\mathrm{CDCl}_{3}\right)$

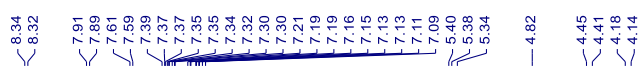<smiles>C=C1CN([125I])c2c(oc3cc(Cl)ccc23)[C@@H](c2ccccc2)[C@@H]1[NH3+]</smiles>

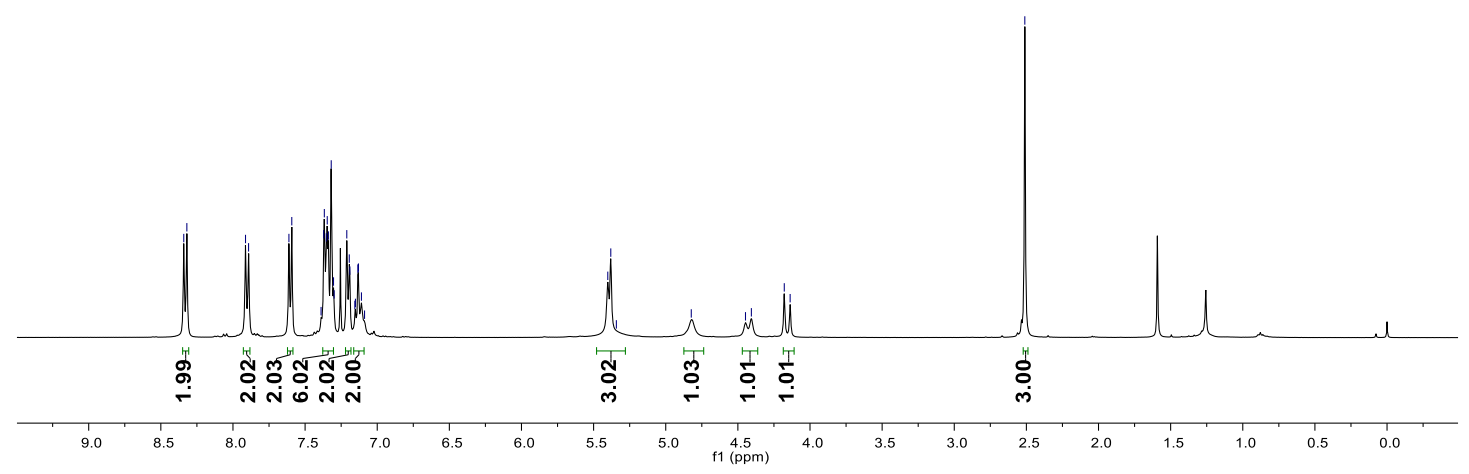

${ }^{13} \mathrm{C}$ NMR spectrum (100 MHz) of product $3 \mathrm{bs}\left(\mathrm{CDCl}_{3}\right)$
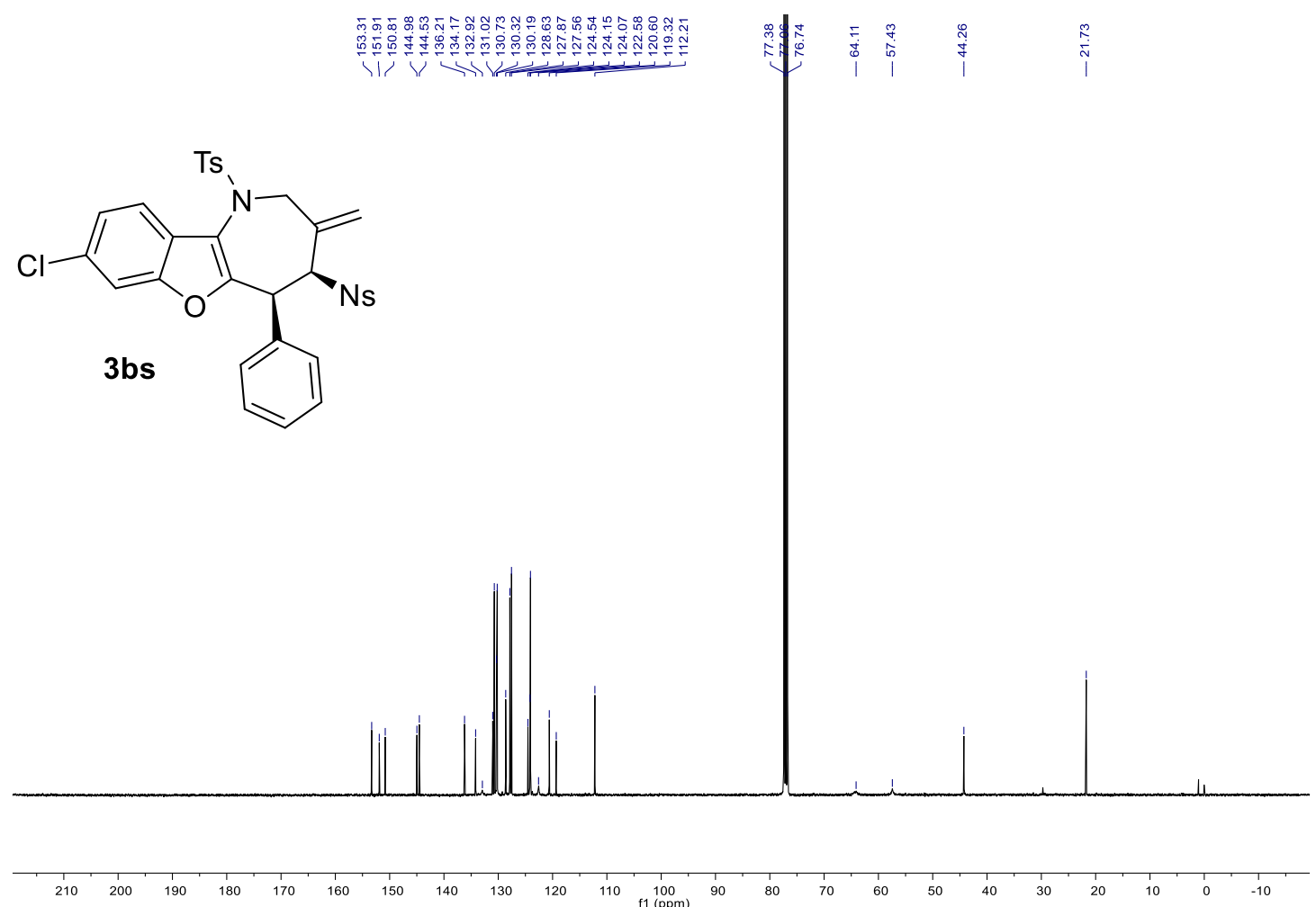
${ }^{1} \mathrm{H}$ NMR spectrum $(400 \mathrm{MHz})$ of product $4 \mathbf{a}\left(\mathrm{CDCl}_{3}\right)$

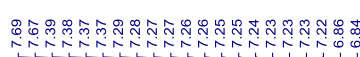

iা

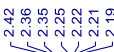<smiles>C=C1C[C@H](c2ccc(Br)cc2)c2oc3ccccc3c2N([12F])C1</smiles>

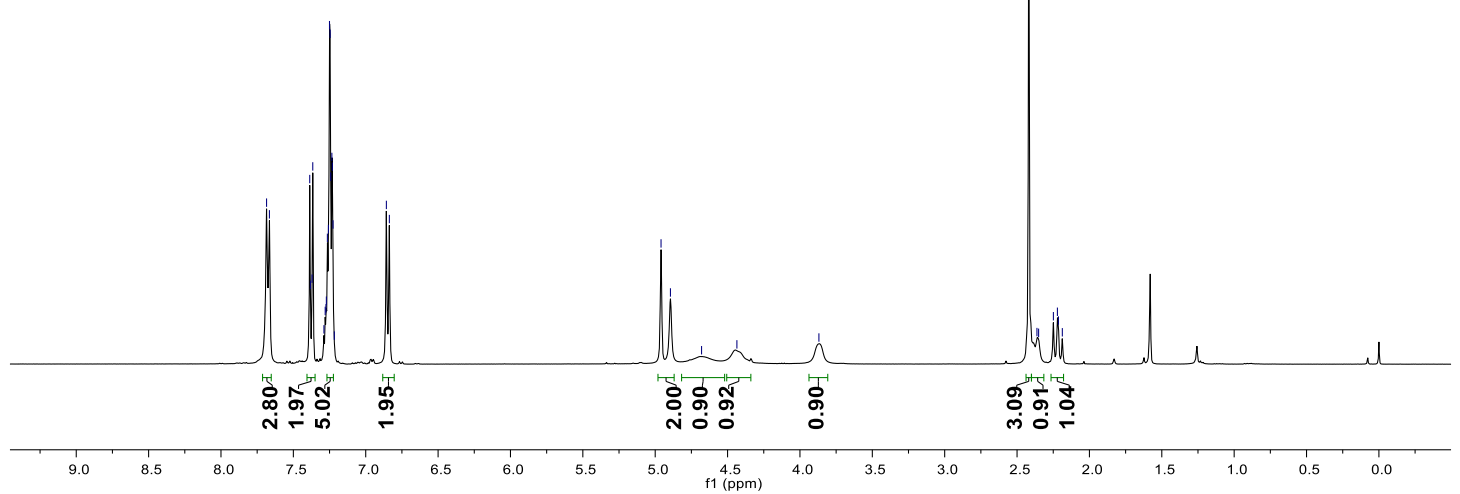

${ }^{13} \mathrm{C}$ NMR spectrum $(100 \mathrm{MHz})$ of product $4 \mathbf{a}\left(\mathrm{CDCl}_{3}\right)$

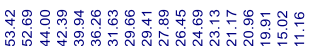

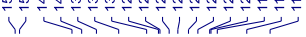

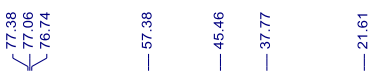<smiles>C=C1C[C@H](c2ccc(Br)cc2)c2oc3ccccc3c2N([As])C1</smiles>

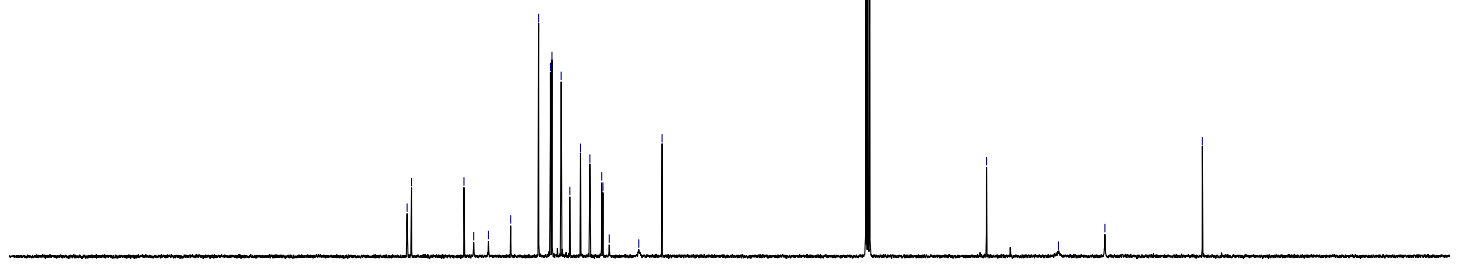

$10 \cos _{11(00 \mathrm{~mm})}^{100} 90$ 
${ }^{1} \mathrm{H}$ NMR spectrum $(400 \mathrm{MHz})$ of product $4 \mathbf{b}\left(\mathrm{CDCl}_{3}\right)$

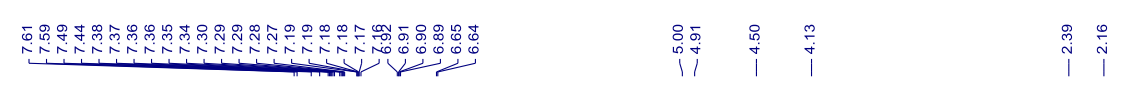

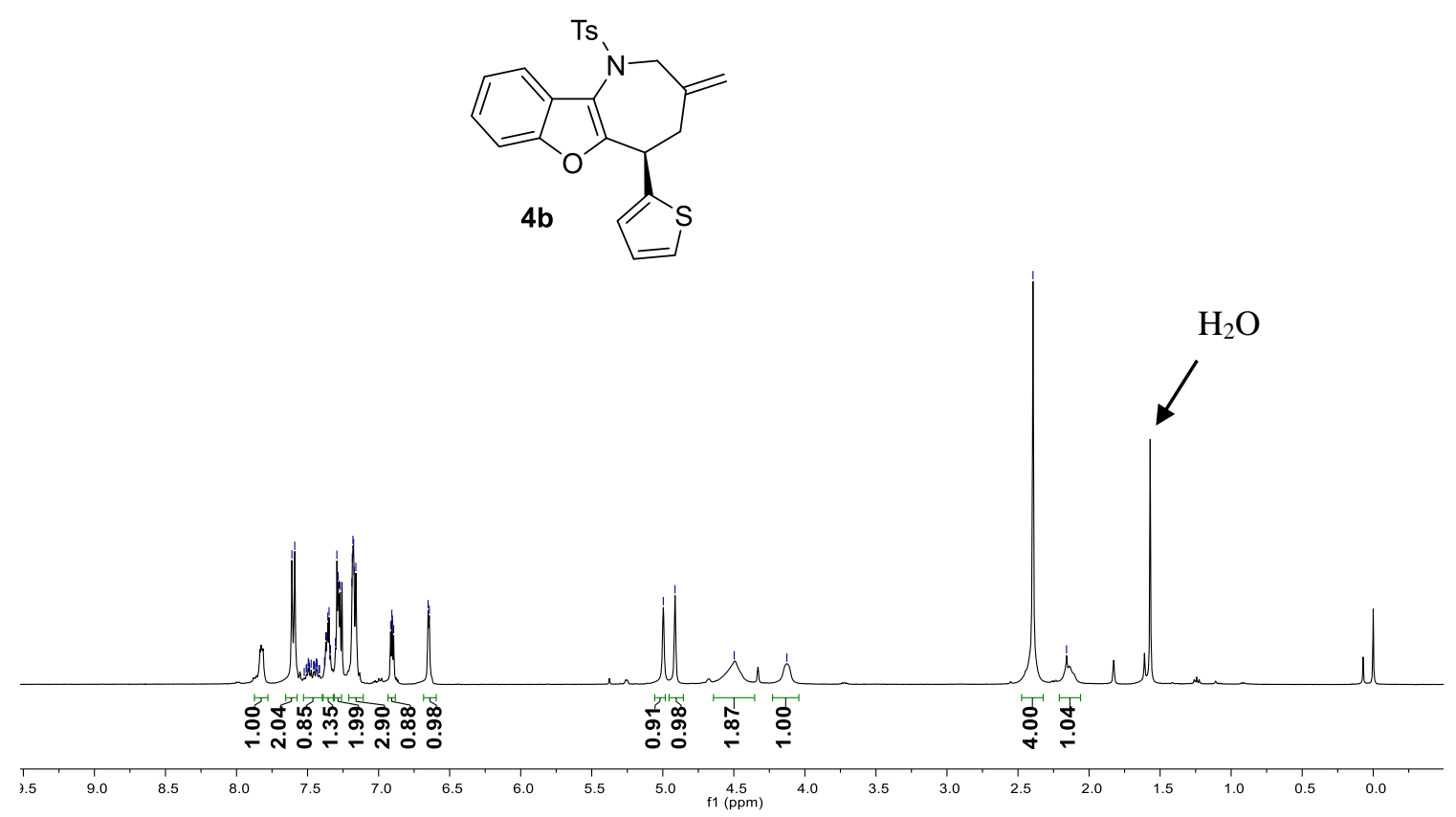

${ }^{13} \mathrm{C}$ NMR spectrum $(100 \mathrm{MHz})$ of product $4 \mathbf{b}\left(\mathrm{CDCl}_{3}\right)$

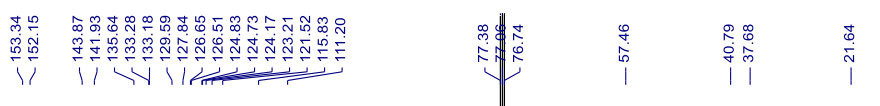<smiles>C=C1C[C@H](c2cccs2)c2oc3ccccc3c2N(C)C1</smiles>

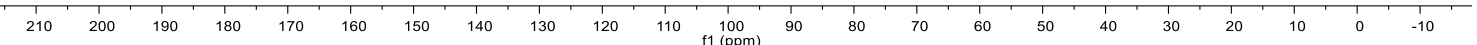


${ }^{1} \mathrm{H}$ NMR spectrum $(400 \mathrm{MHz})$ of product $5\left(\mathrm{CDCl}_{3}\right)$

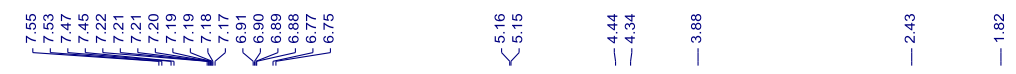

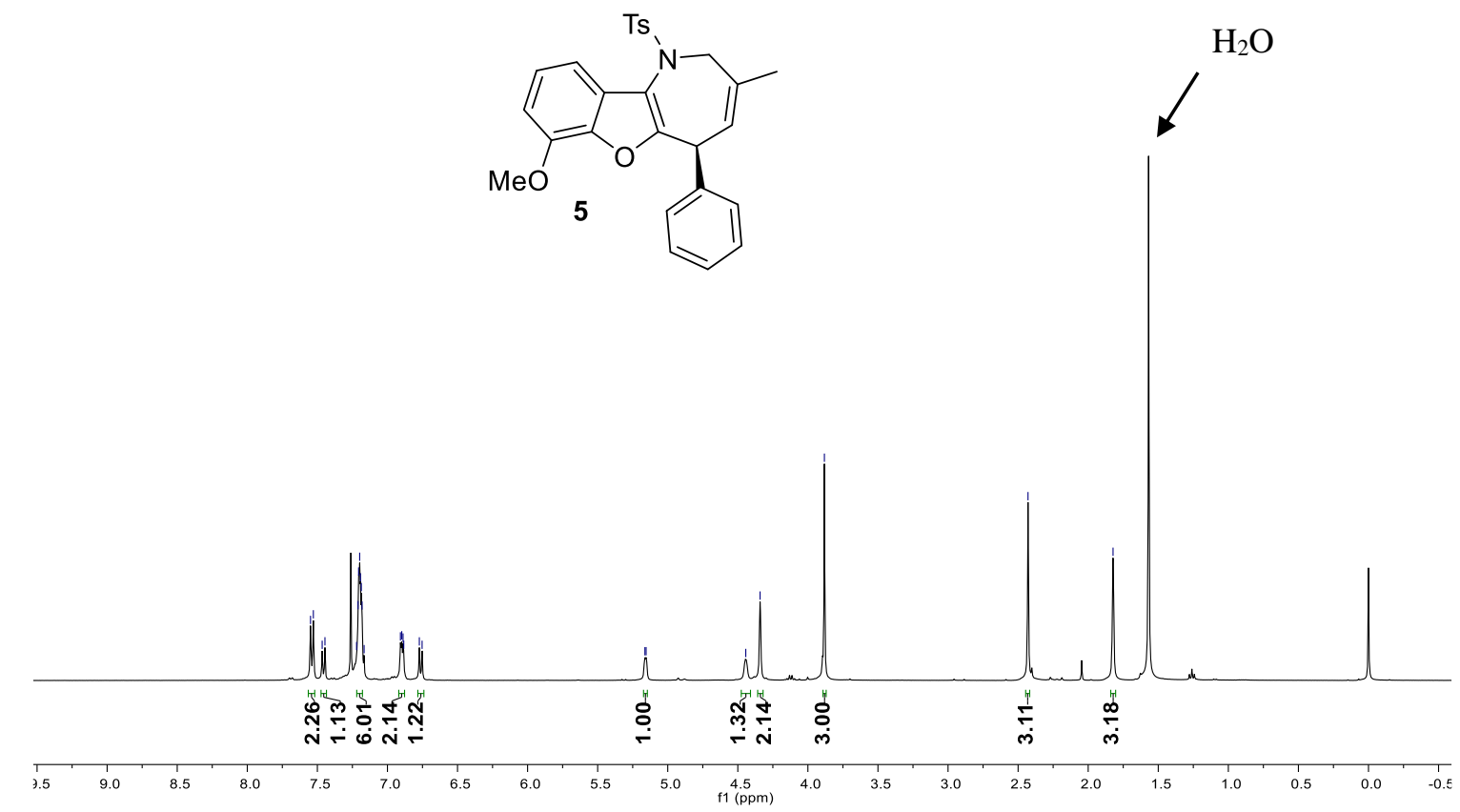

$\mathrm{H}_{2} \mathrm{O}$

${ }^{13} \mathrm{C}$ NMR spectrum $(100 \mathrm{MHz})$ of product $5\left(\mathrm{CDCl}_{3}\right)$

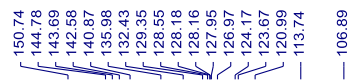

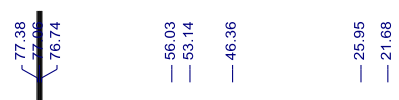<smiles>COc1cccc2c3c(oc12)C(c1ccccc1)C=C(C)CN3[13CH3]</smiles>

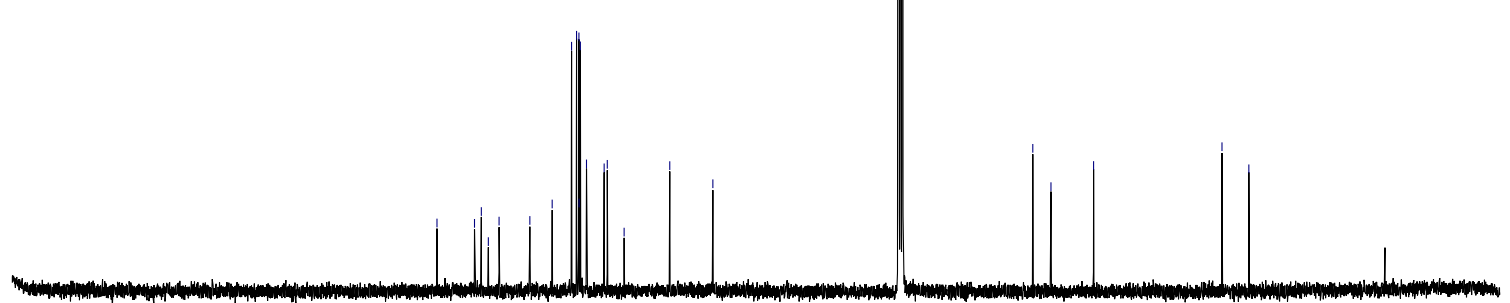

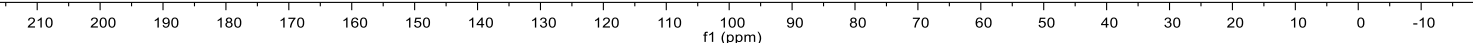


${ }^{1} \mathrm{H}$ NMR spectrum $(400 \mathrm{MHz})$ of product 6 (Acetone- $d_{6}$ )

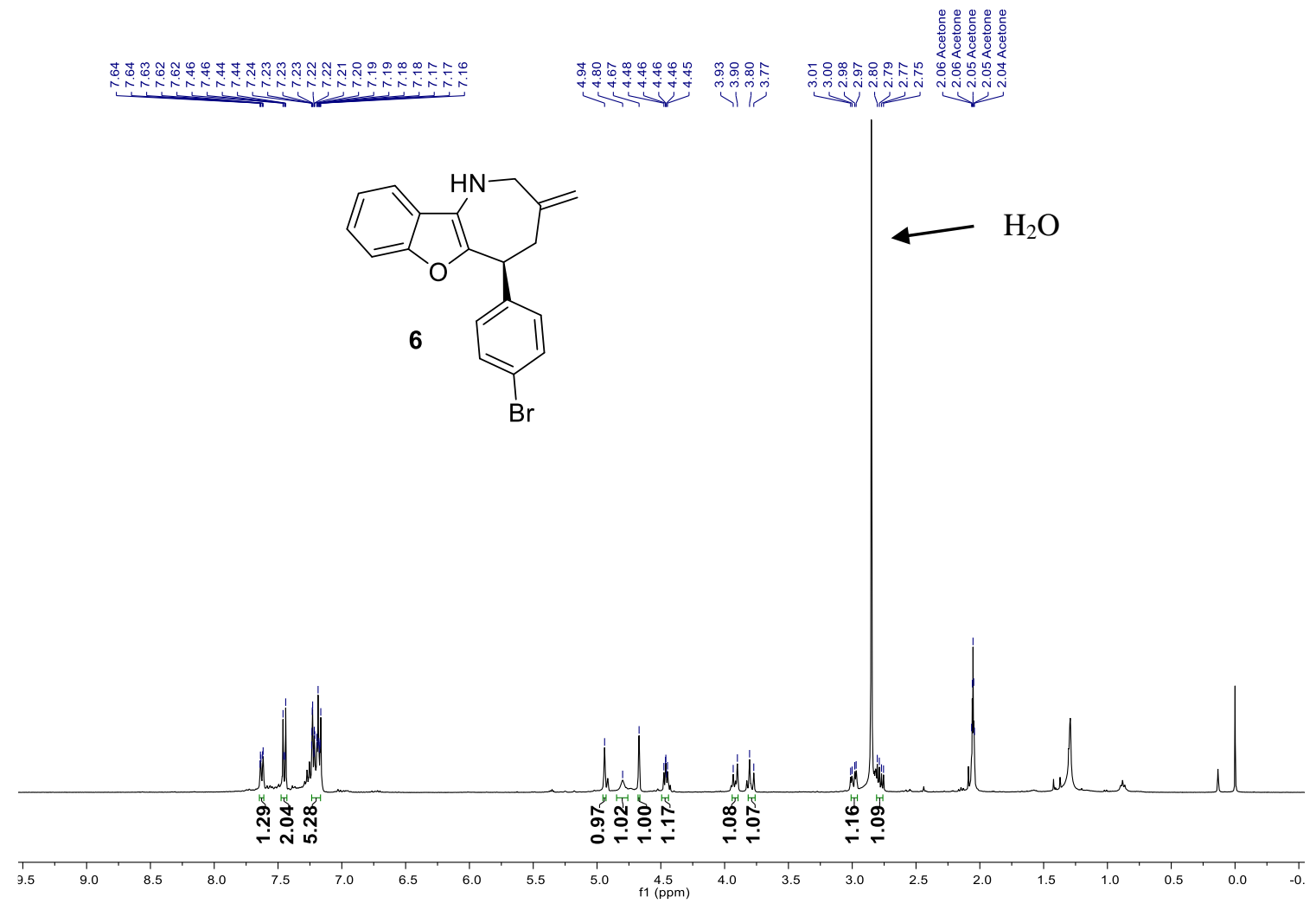

${ }^{13} \mathrm{C}$ NMR spectrum (100 MHz) of product 6 (Acetone- $\left.d_{6}\right)$

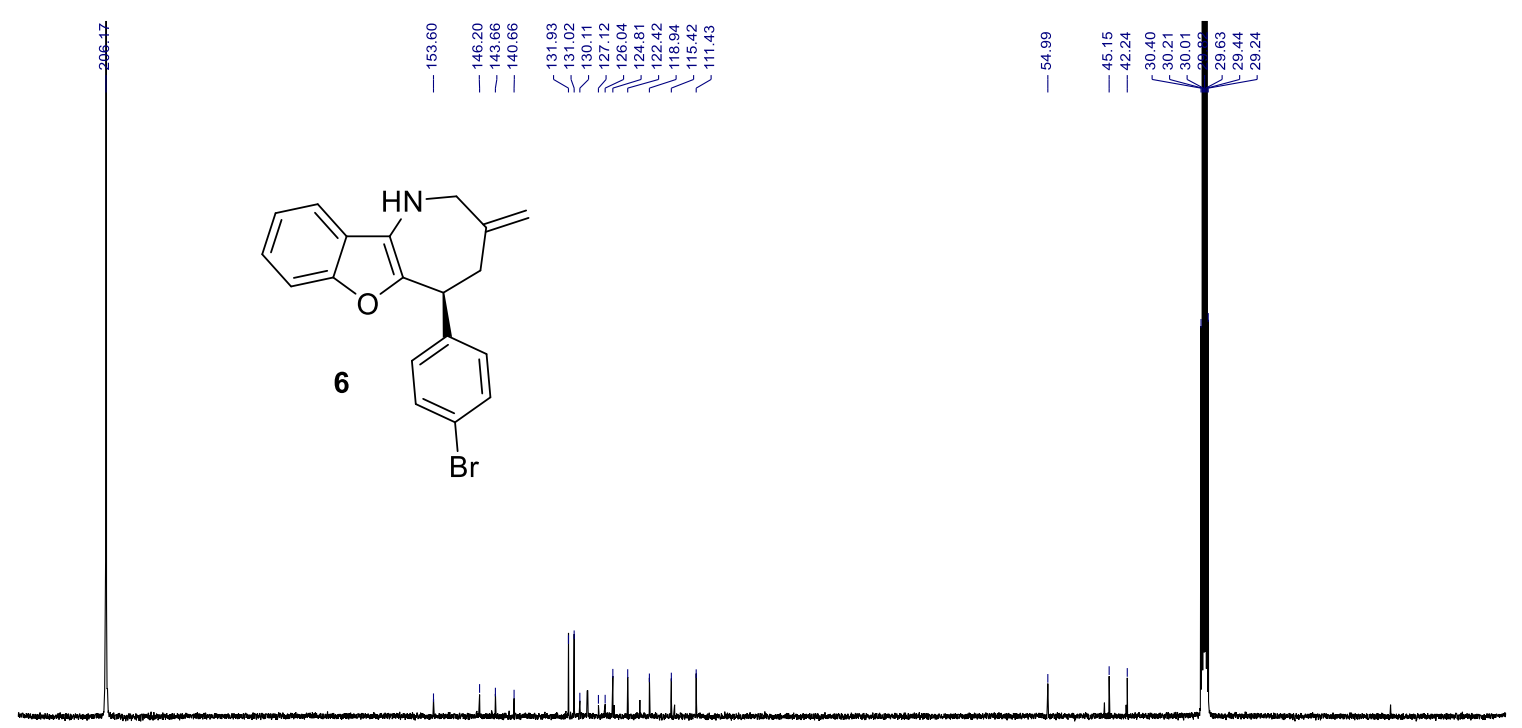

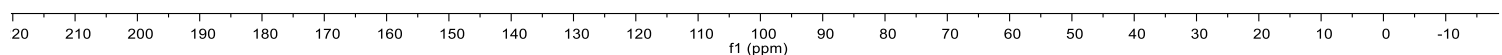




\section{Chiral HPLC Chromatograms}<smiles>C=C1CN([As])c2c(oc3ccccc23)C(c2ccccc2)C1[N+](=O)[O-]</smiles>

3 ba
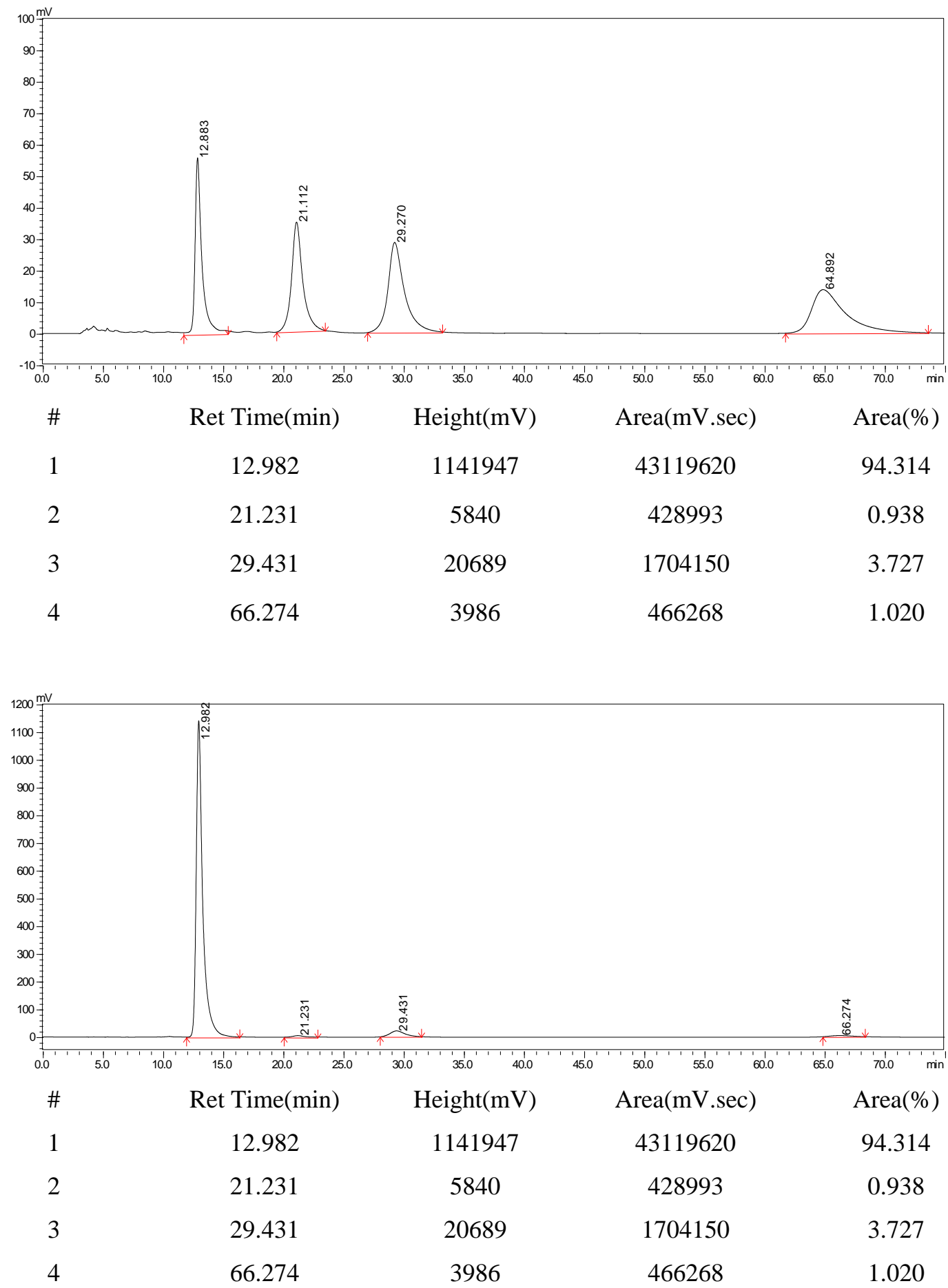
<smiles>C=C1CN([As])c2c(oc3ccccc23)[C@@H](c2ccc(Cl)cc2)[C@@H]1N(C)[SH3+]</smiles>
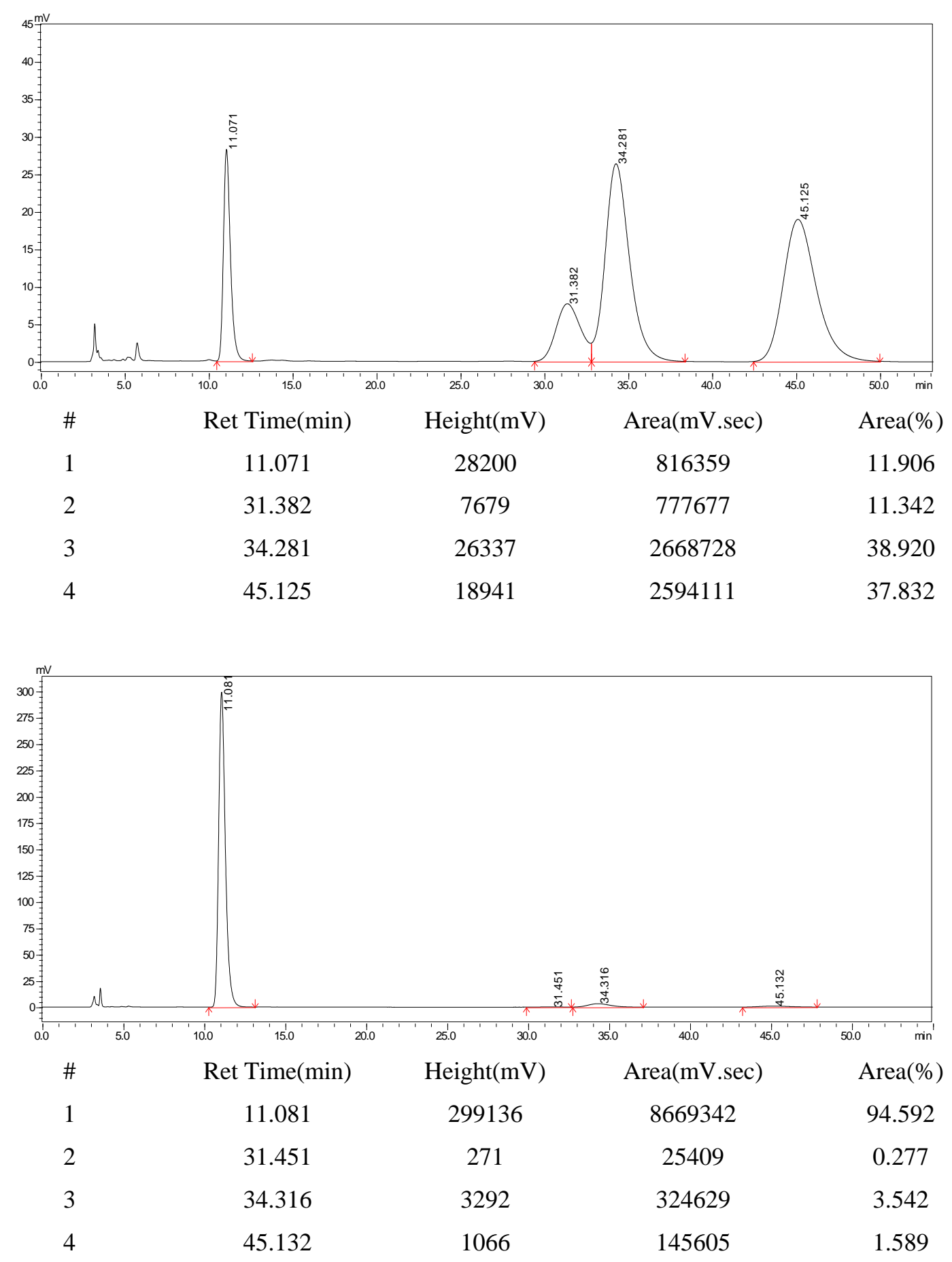

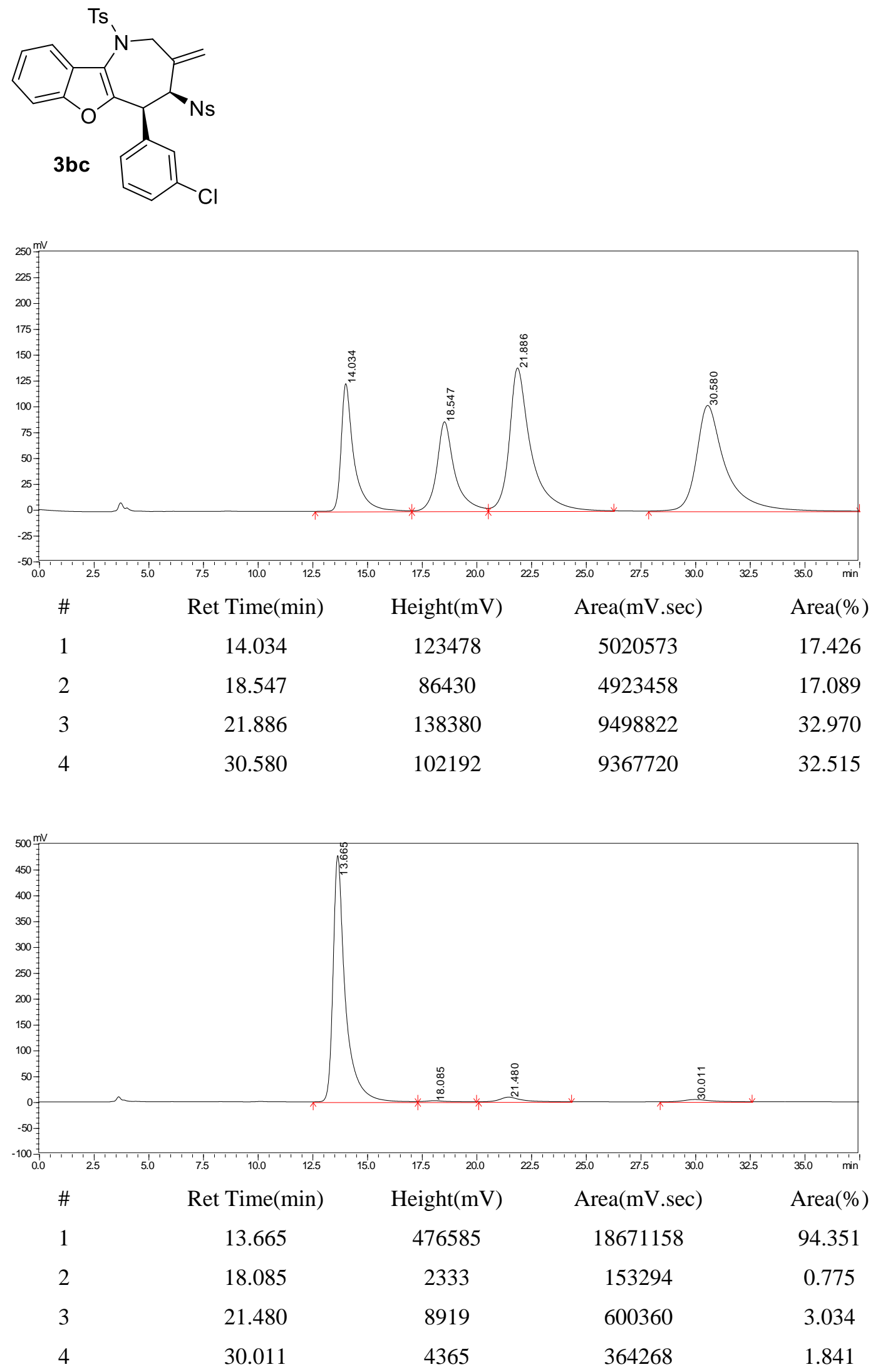

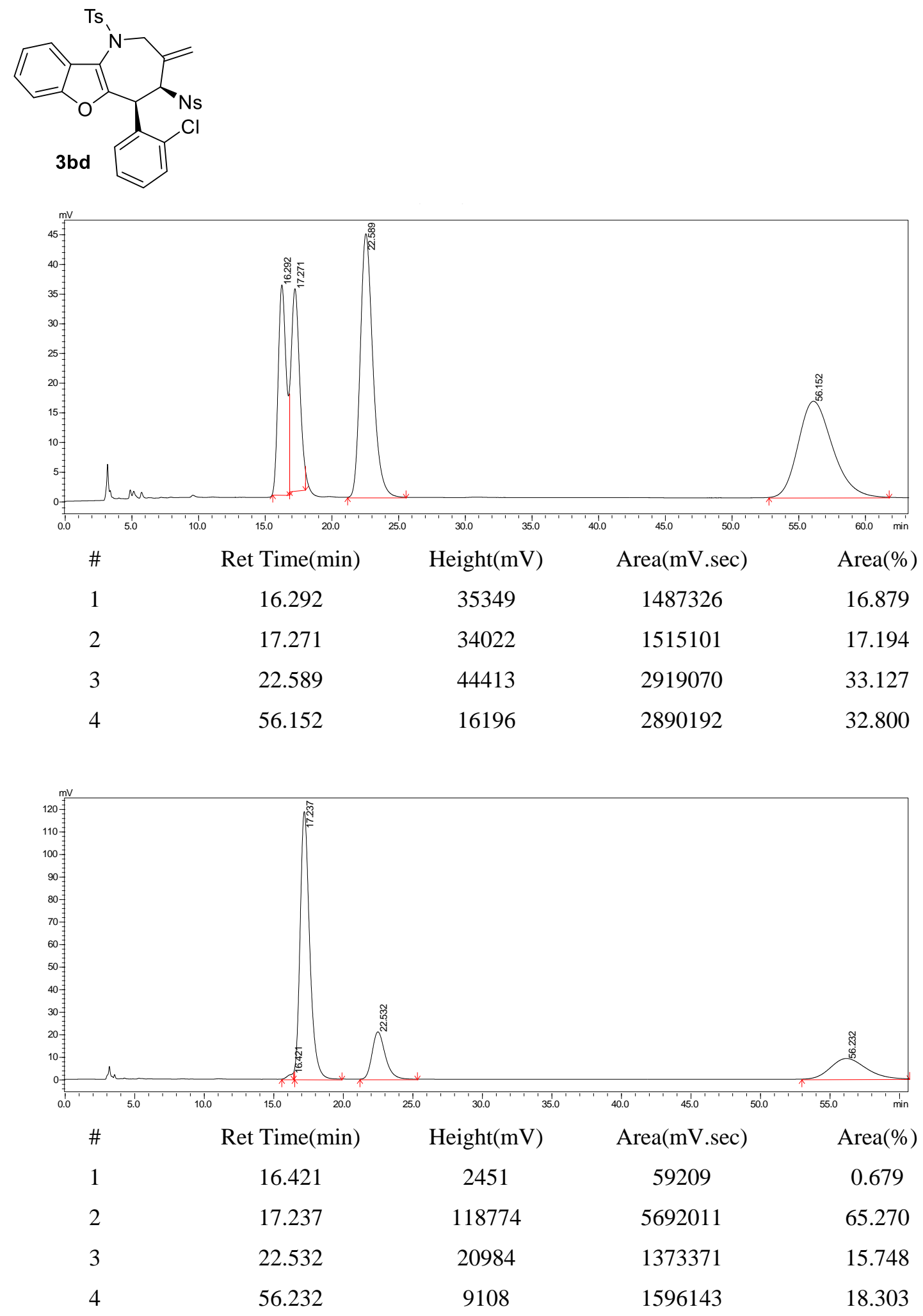

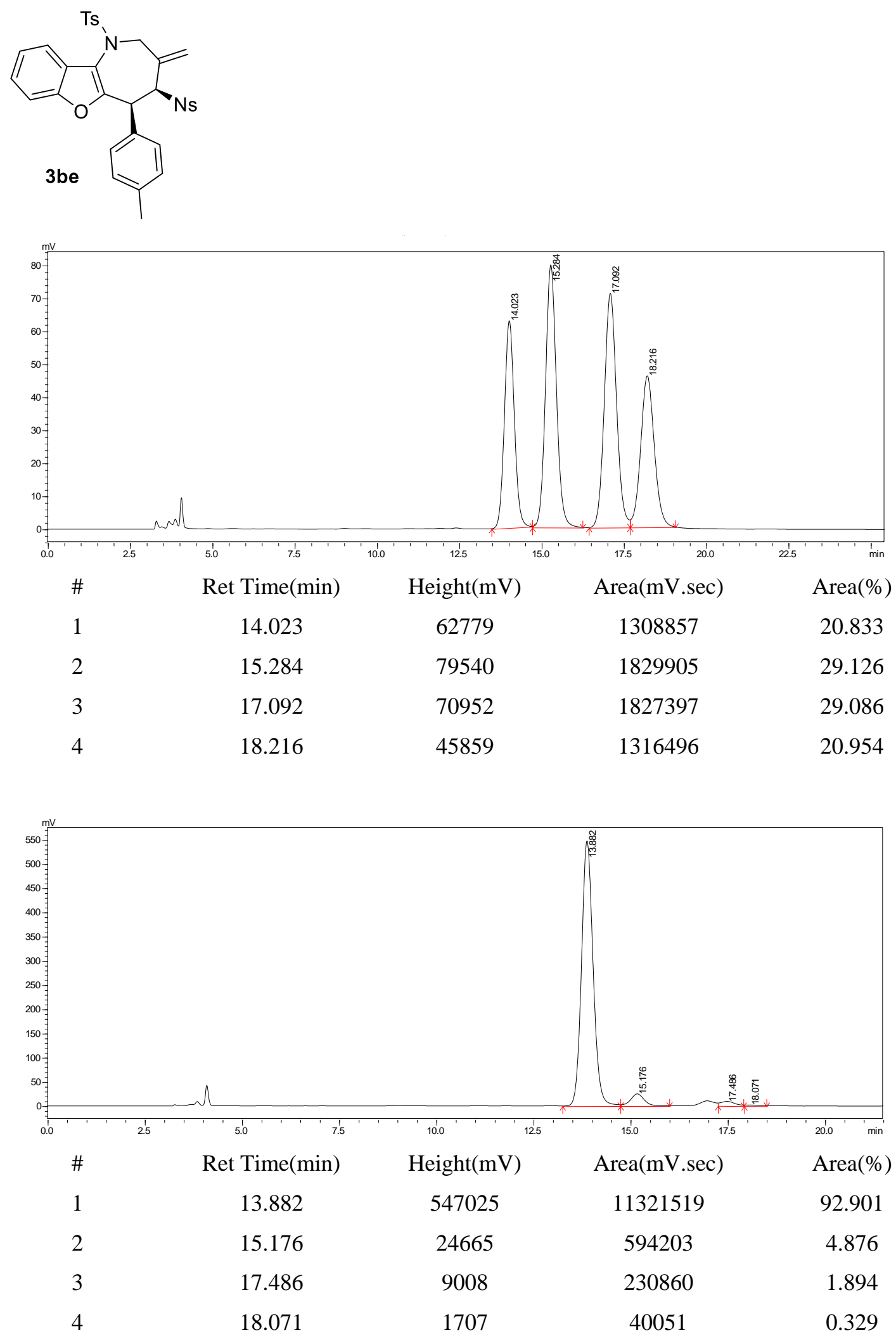

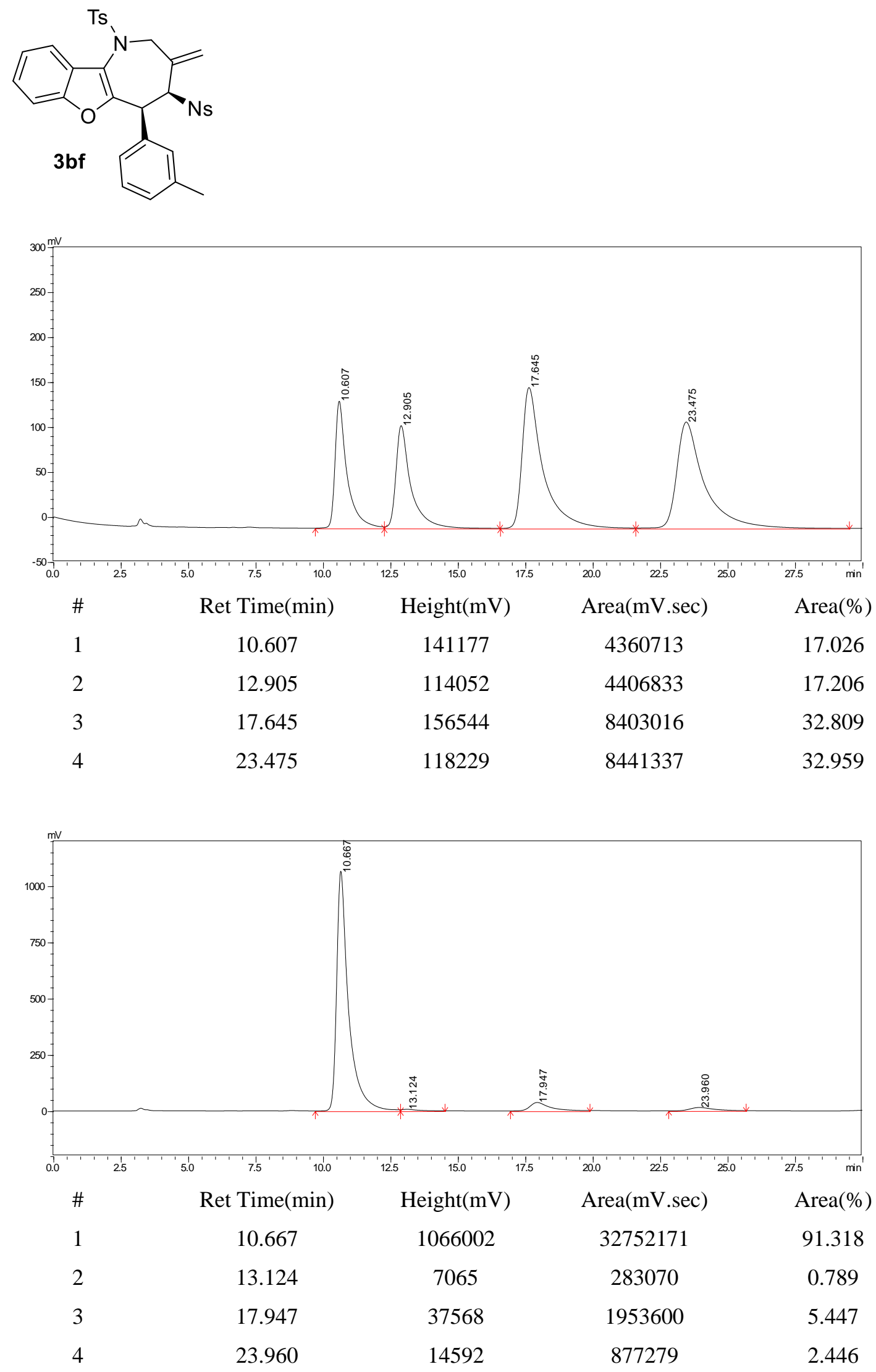

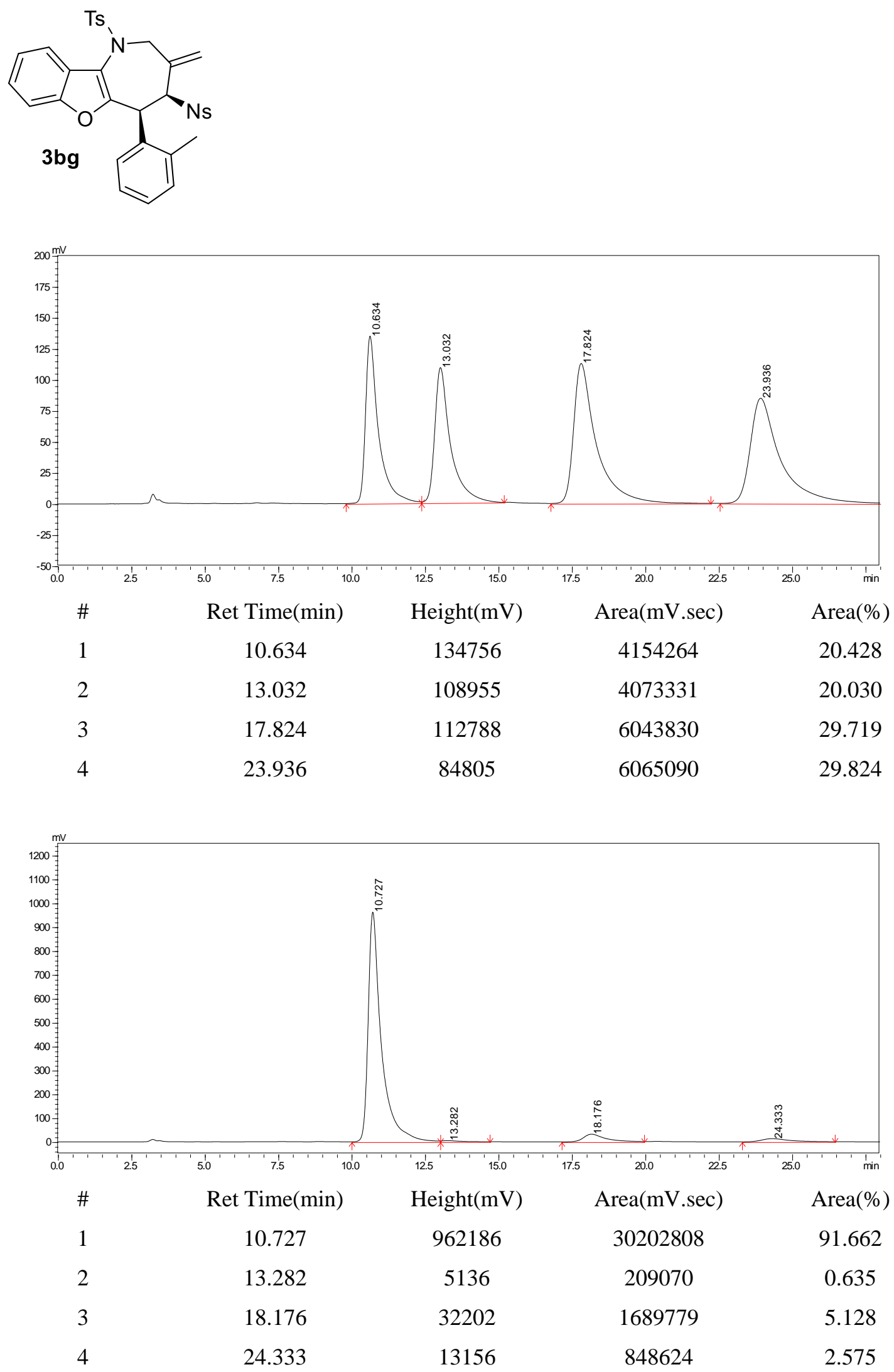

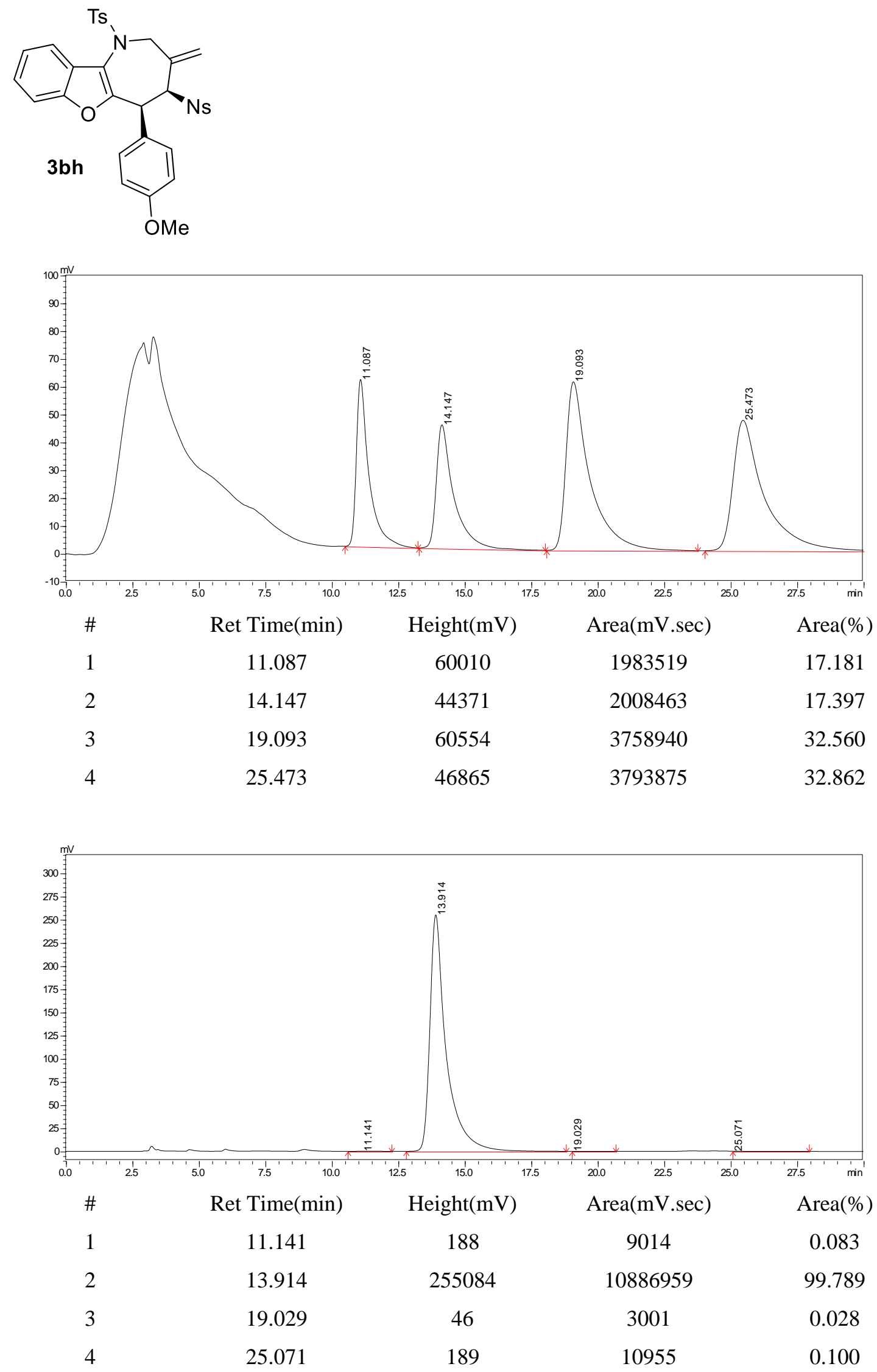

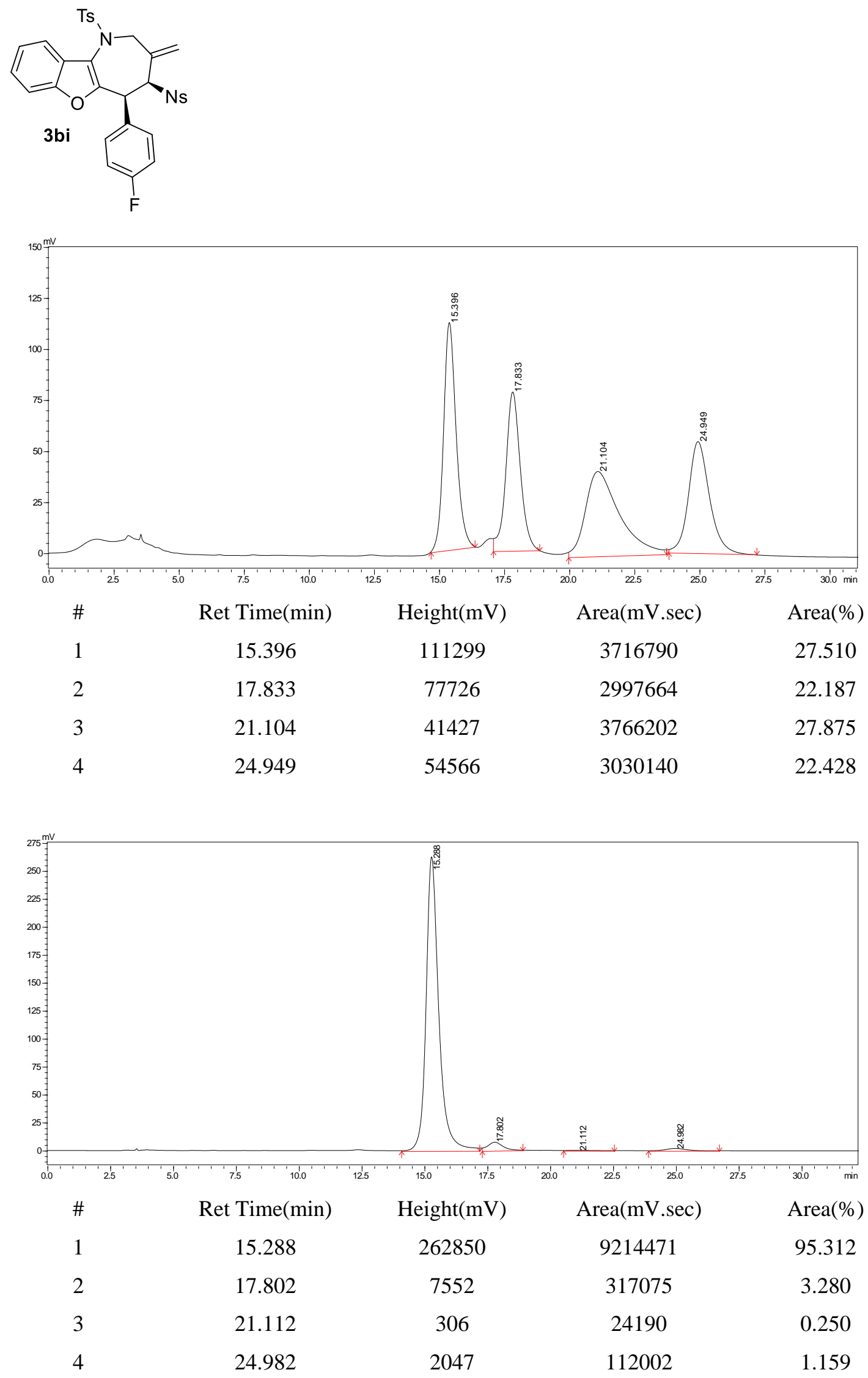

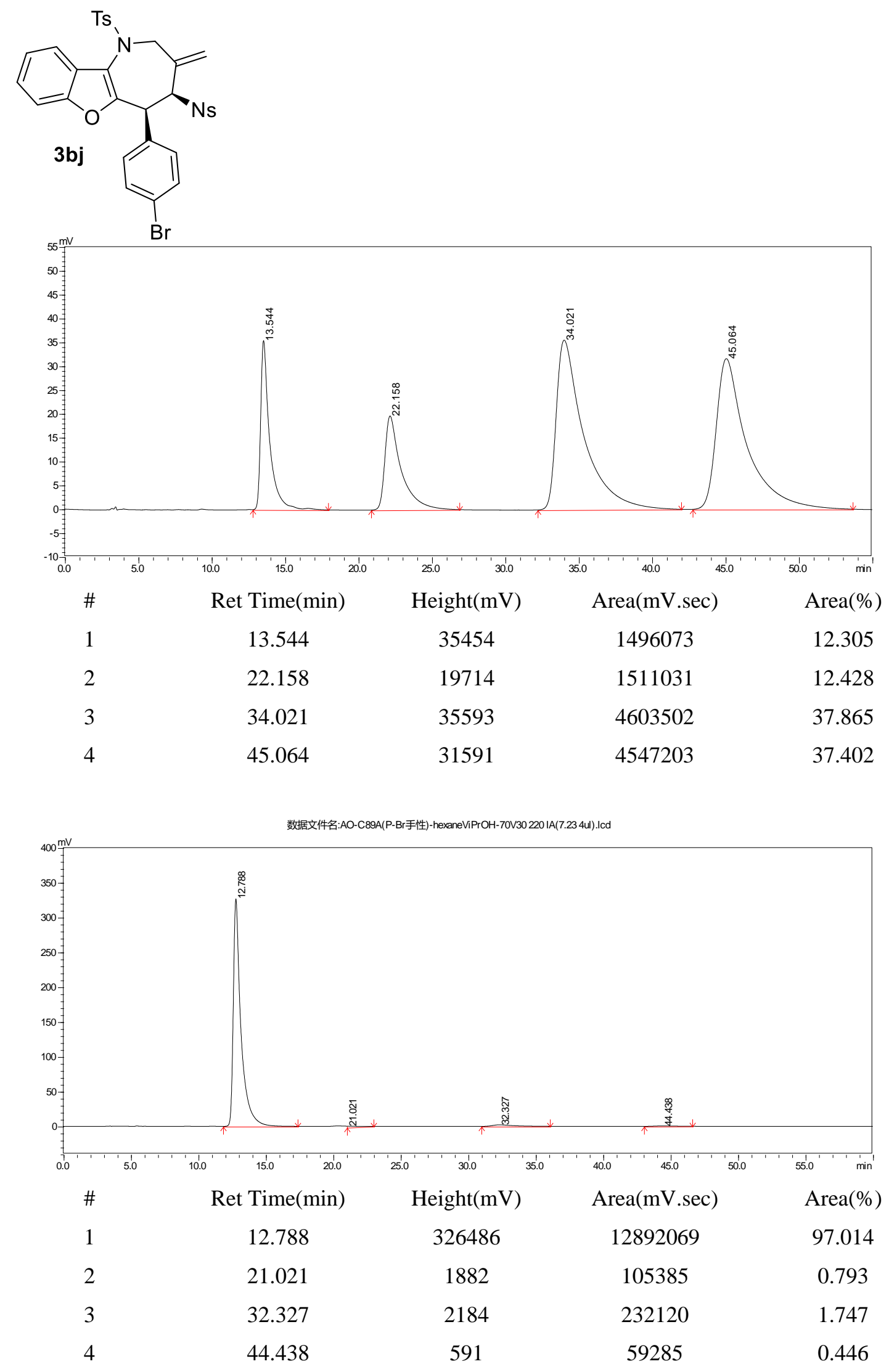

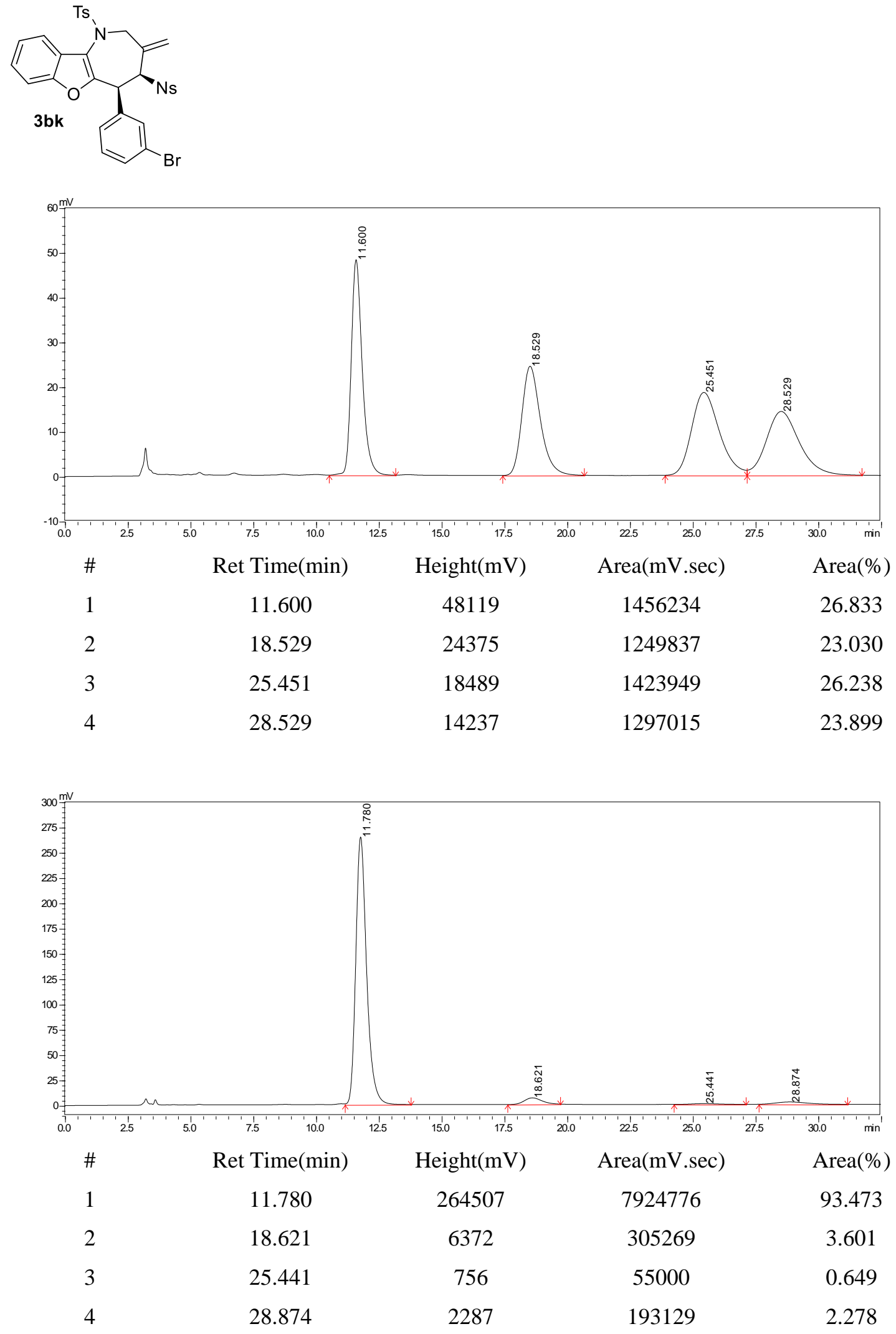

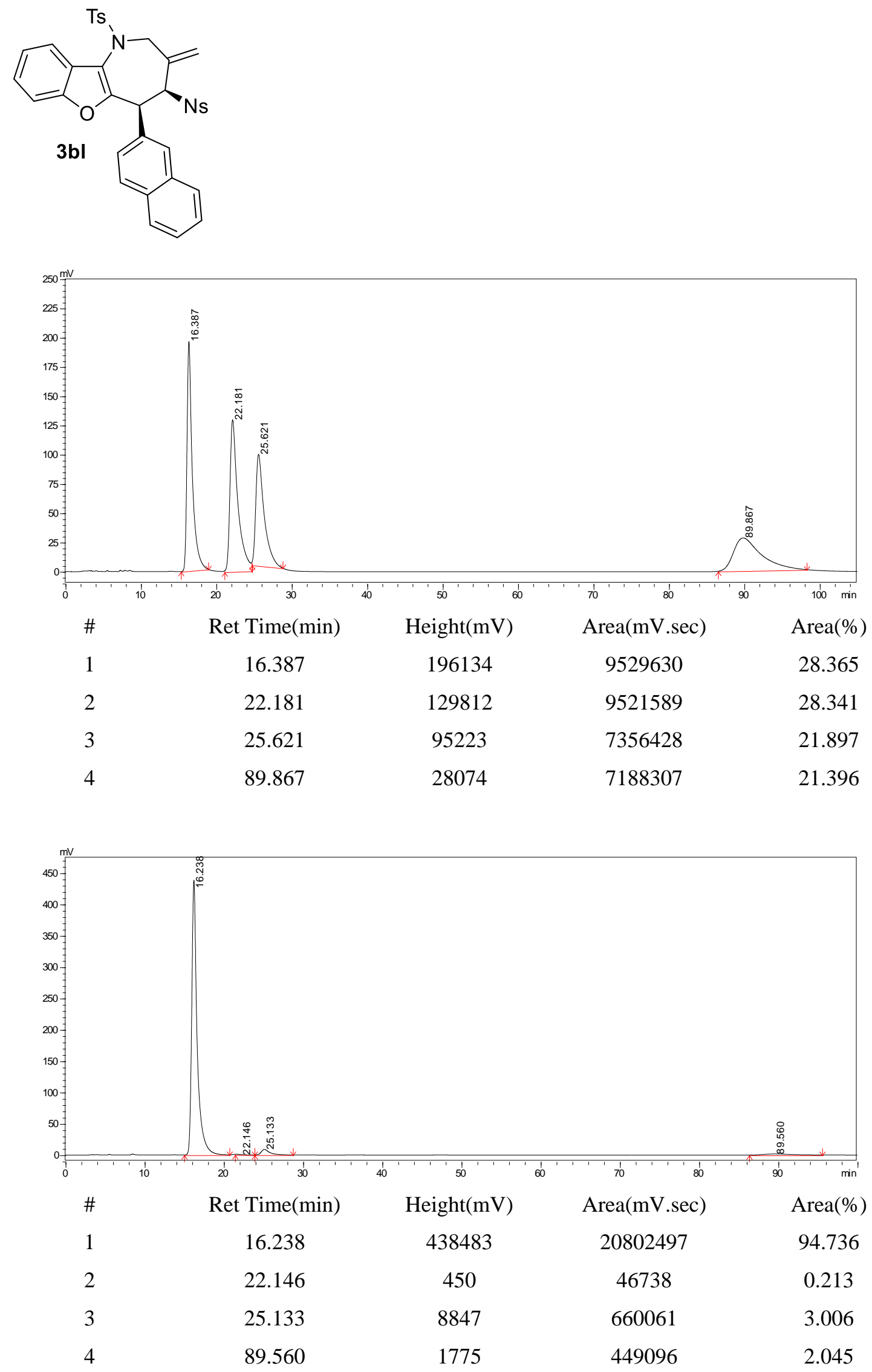

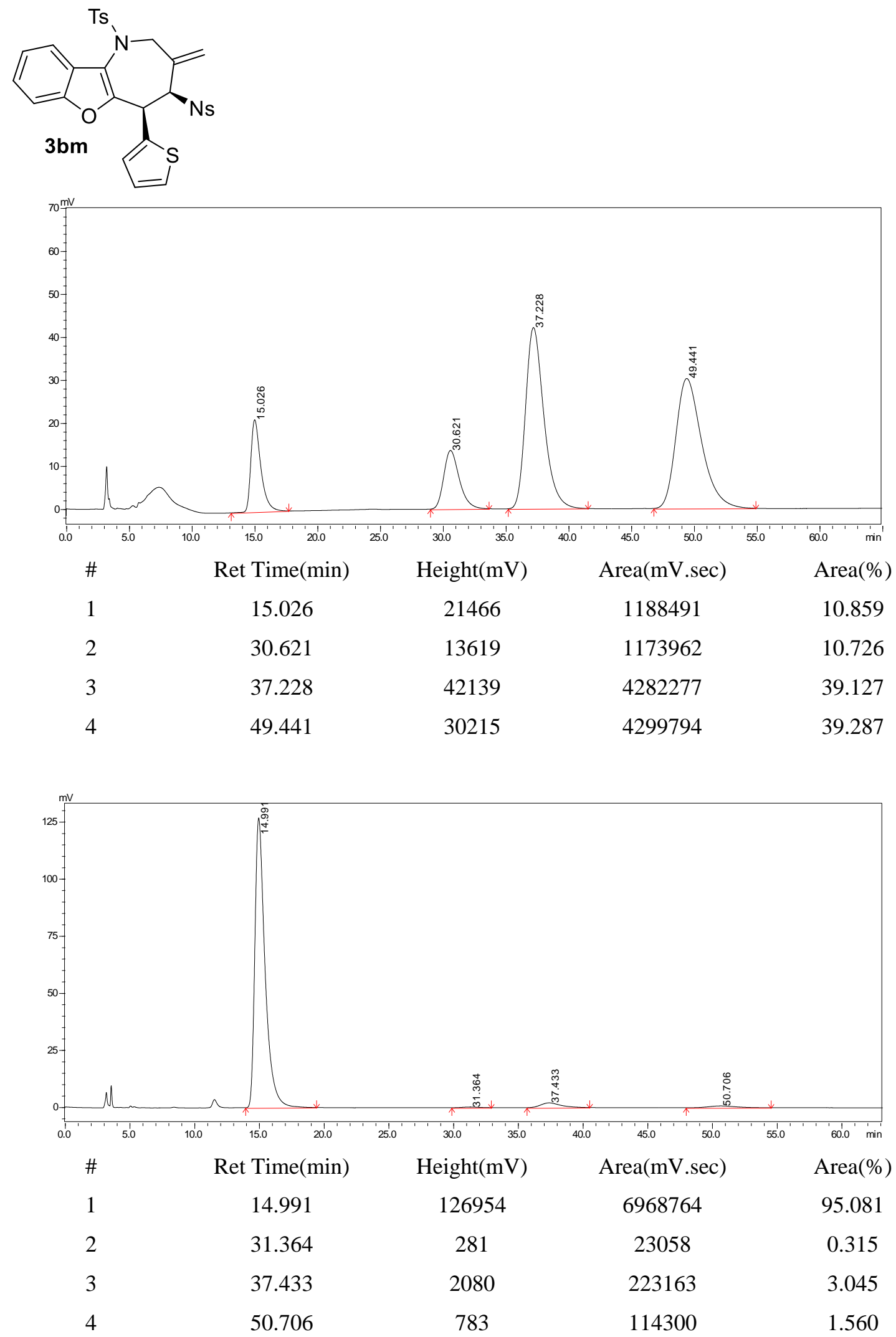

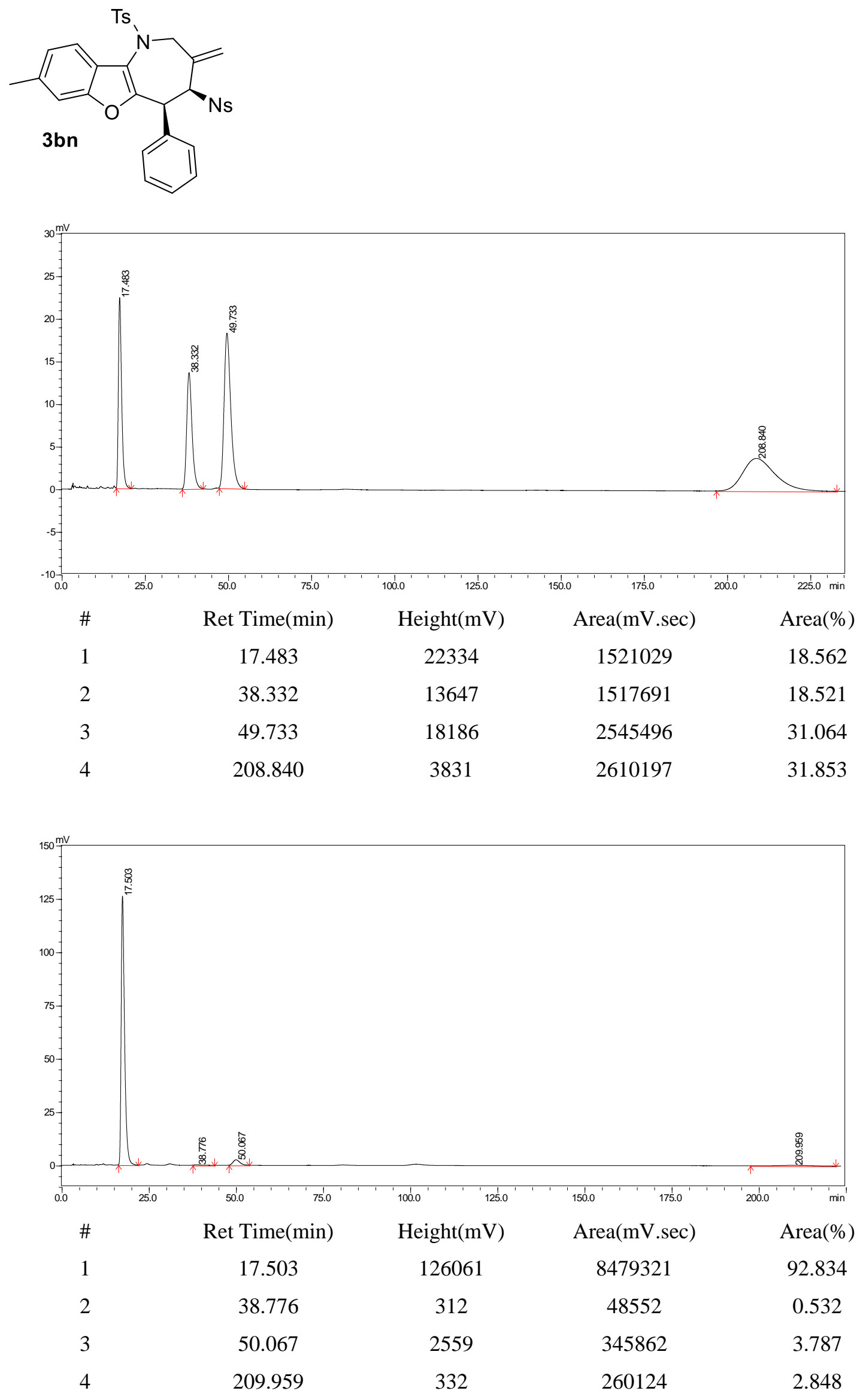

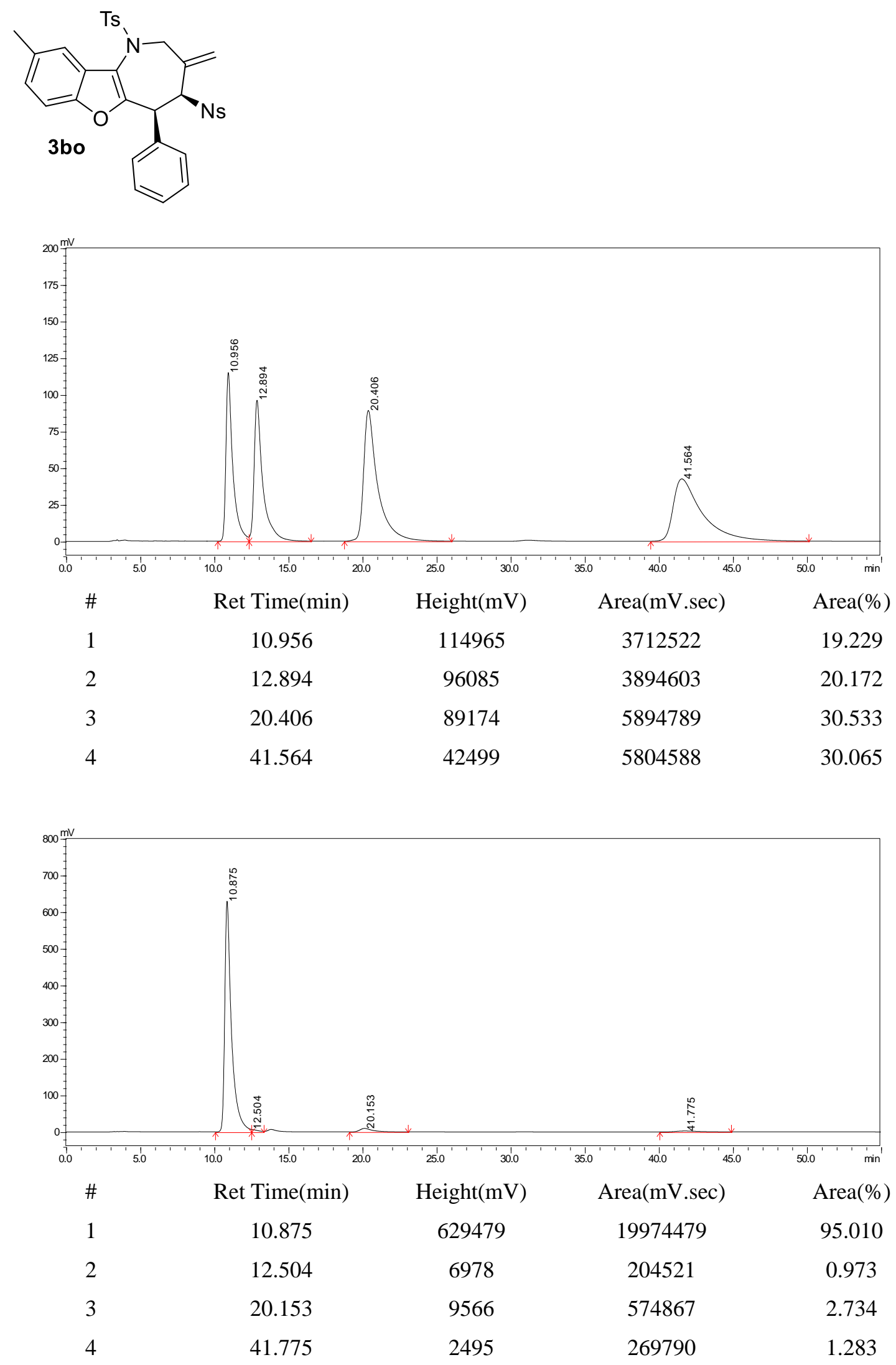

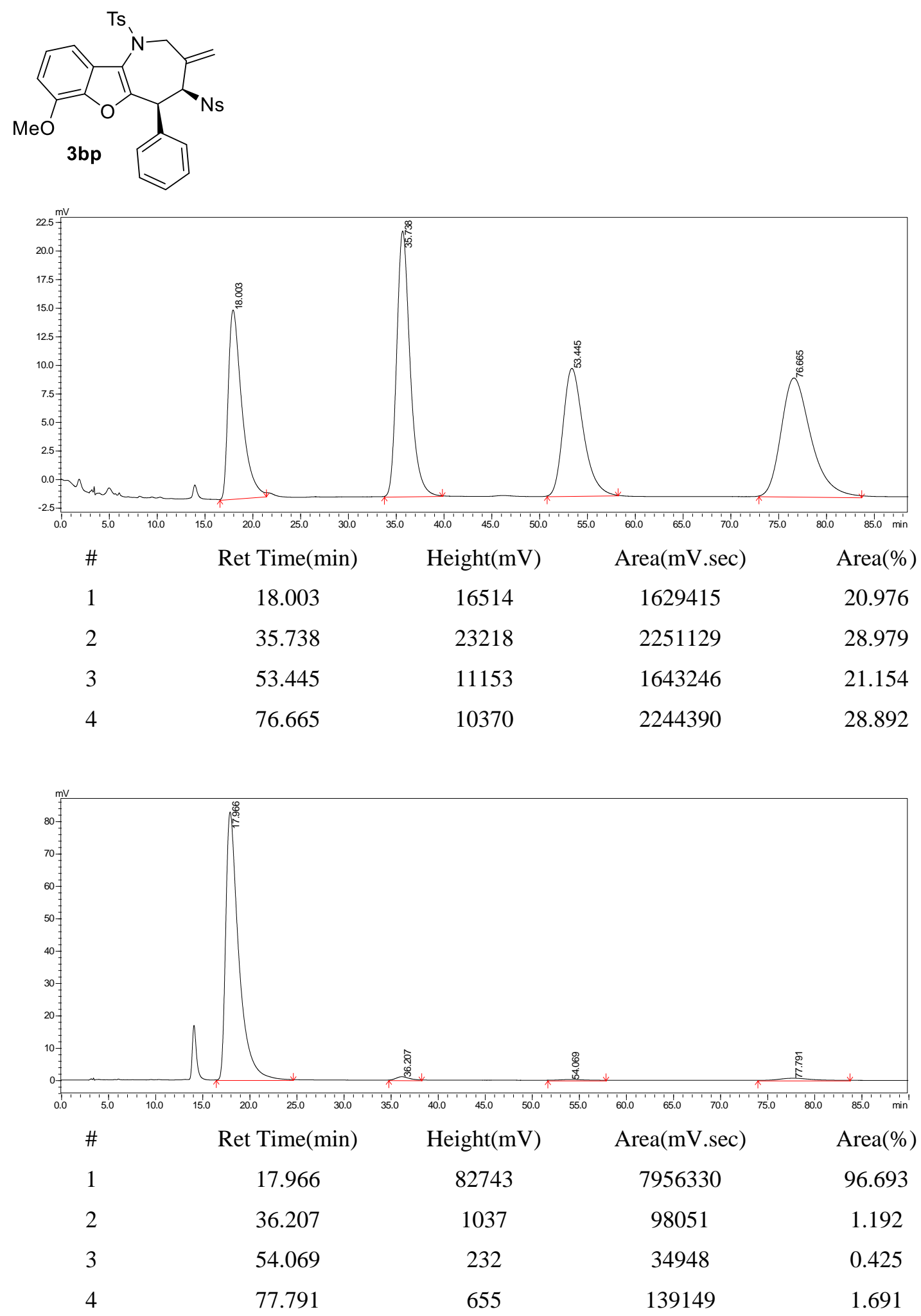

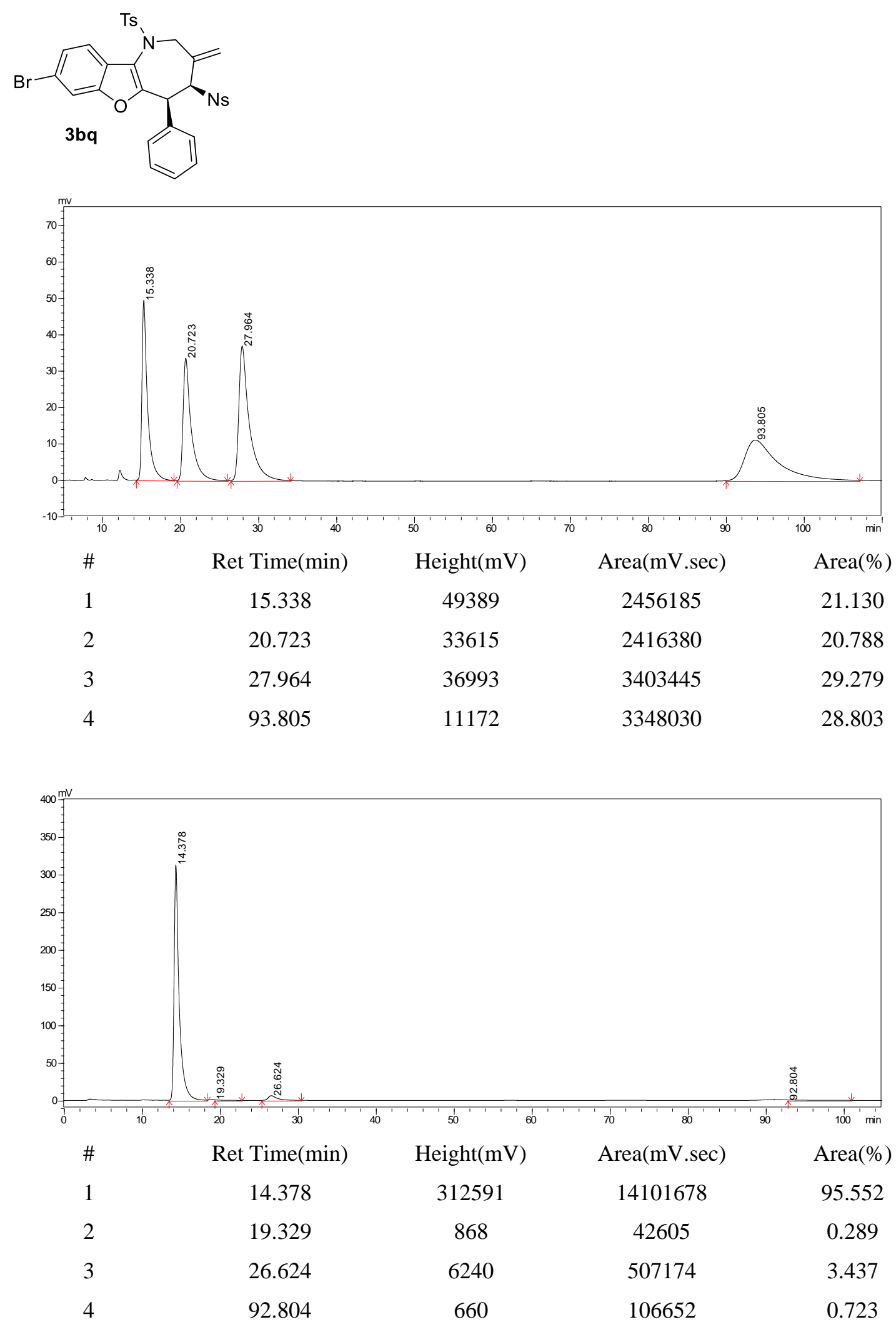

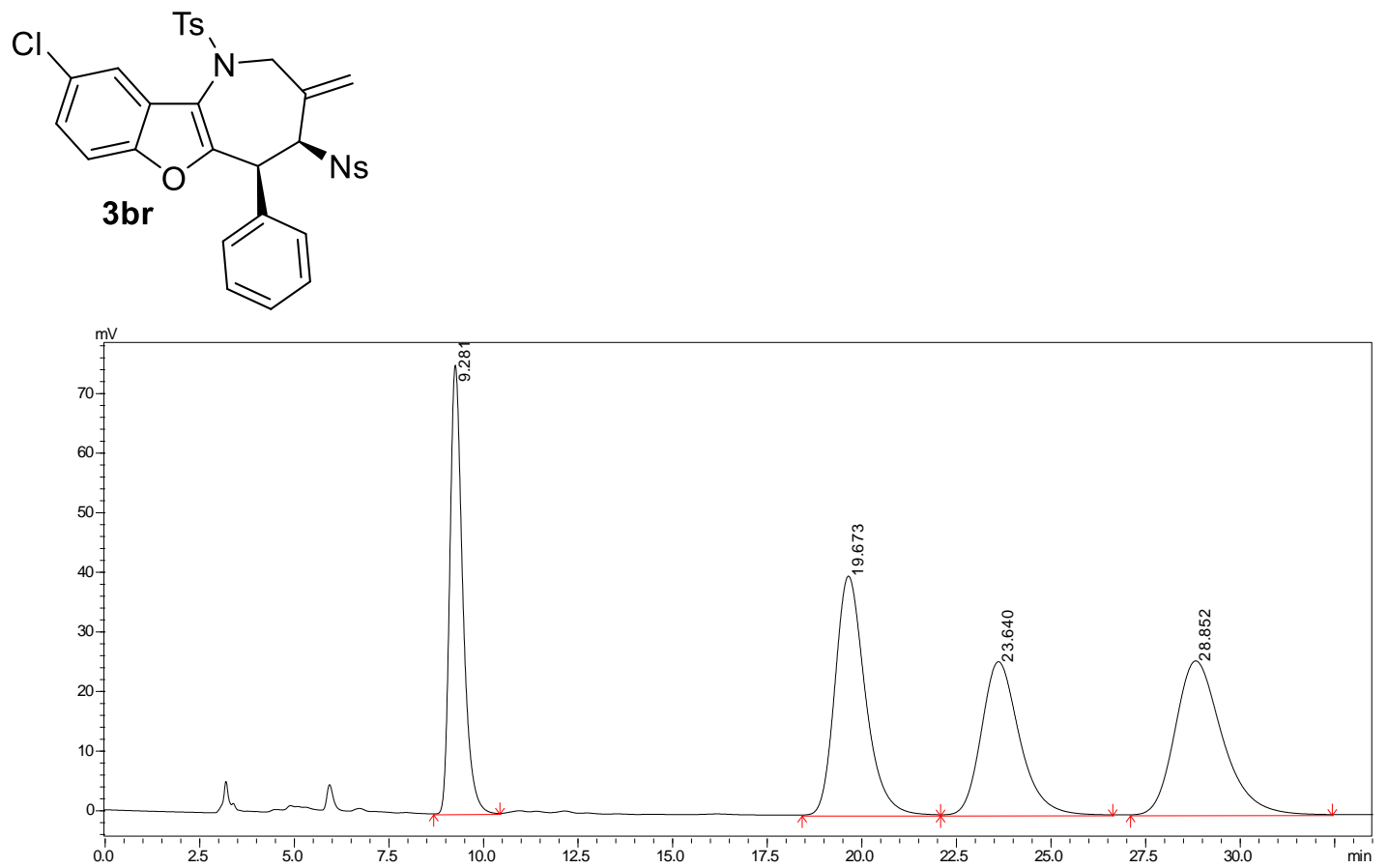

$\begin{array}{ccccc}\# & \text { Ret Time }(\mathrm{min}) & \text { Height }(\mathrm{mV}) & \text { Area }(\mathrm{mV} . \mathrm{sec}) & \text { Area(\%) } \\ 1 & 9.281 & 75277 & 1793342 & 22.313 \\ 2 & 19.673 & 40088 & 2221235 & 27.637 \\ 3 & 23.640 & 25708 & 1793533 & 22.315 \\ 4 & 28.852 & 25816 & 2229093 & 27.735\end{array}$

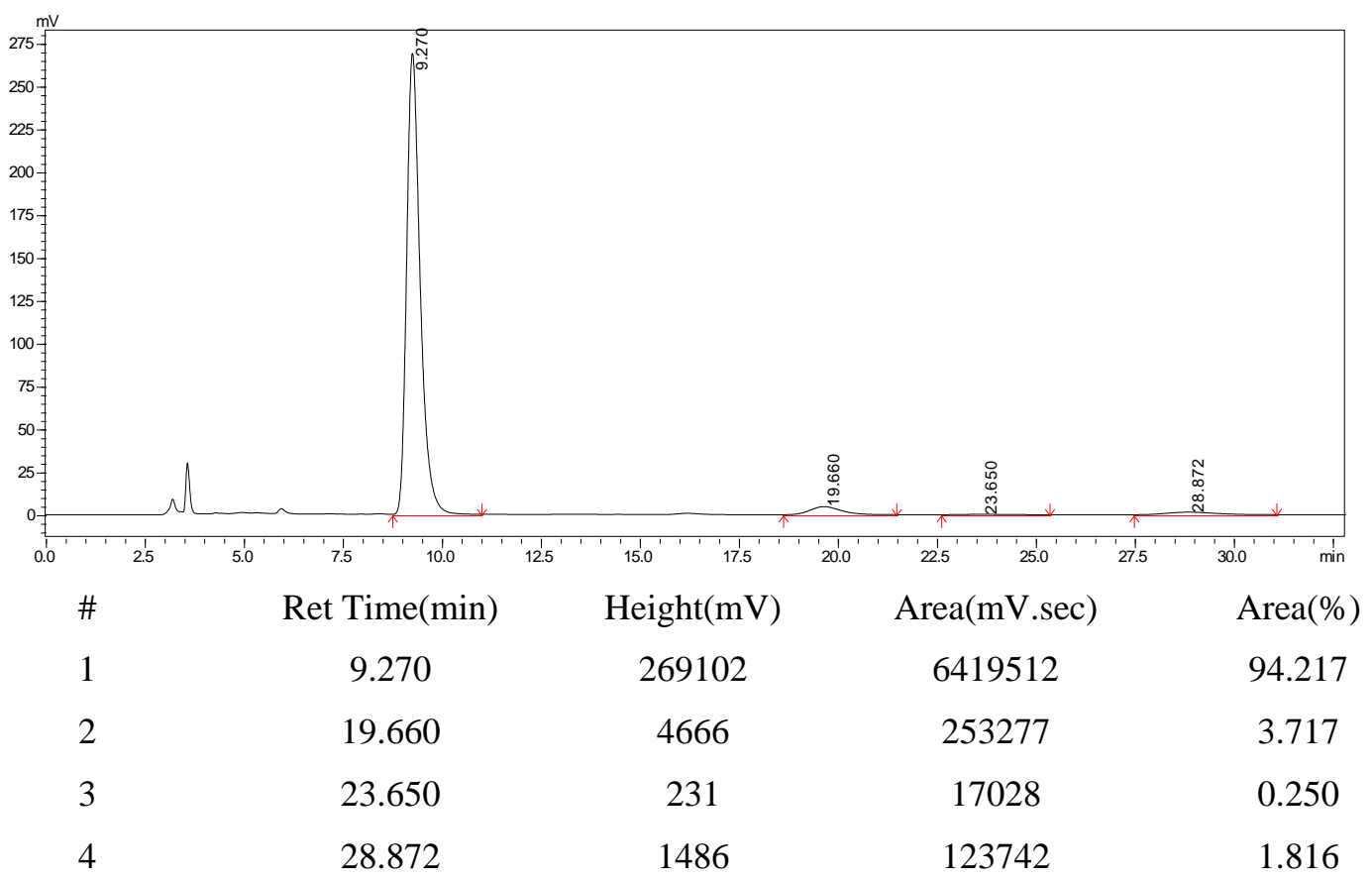



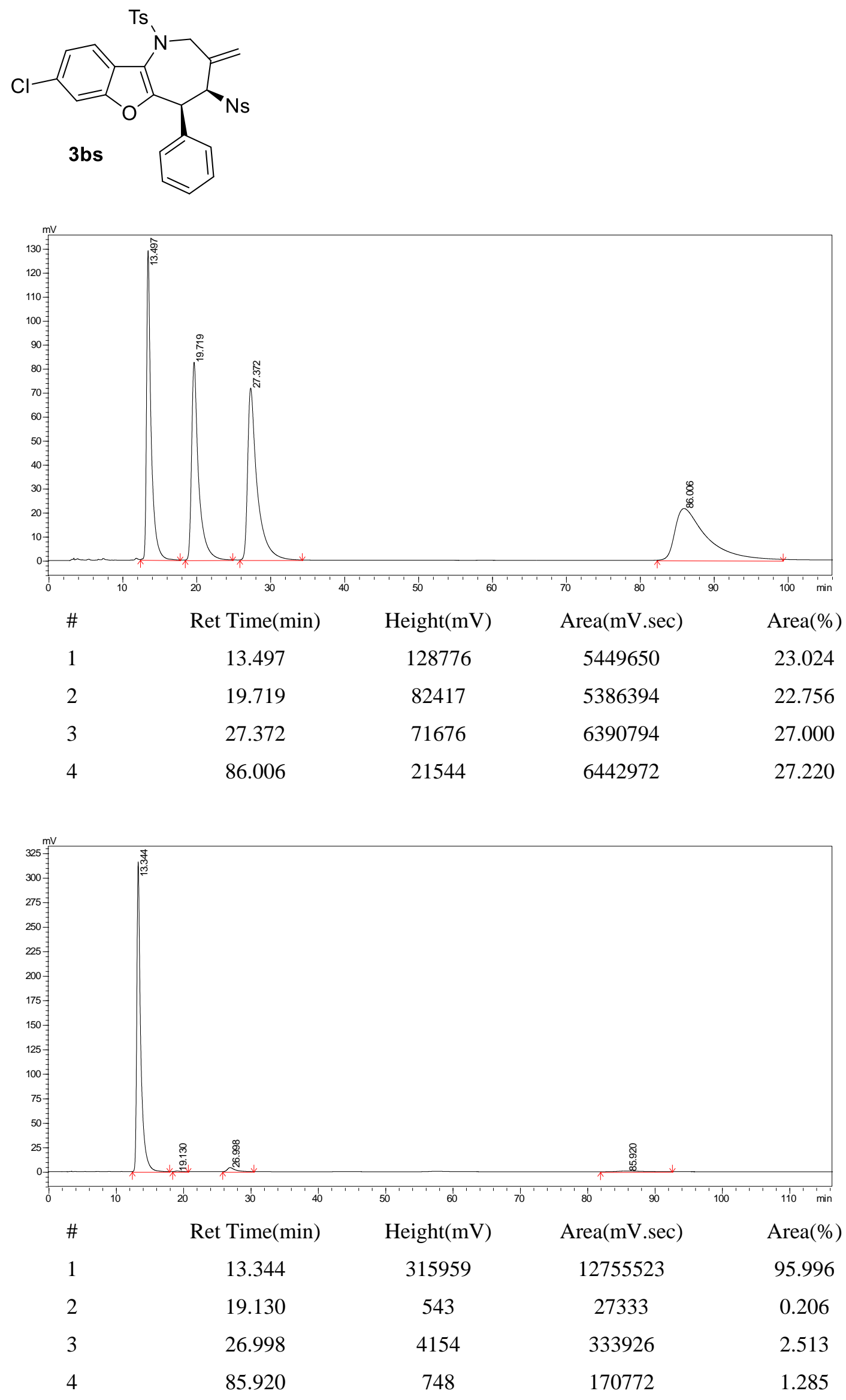

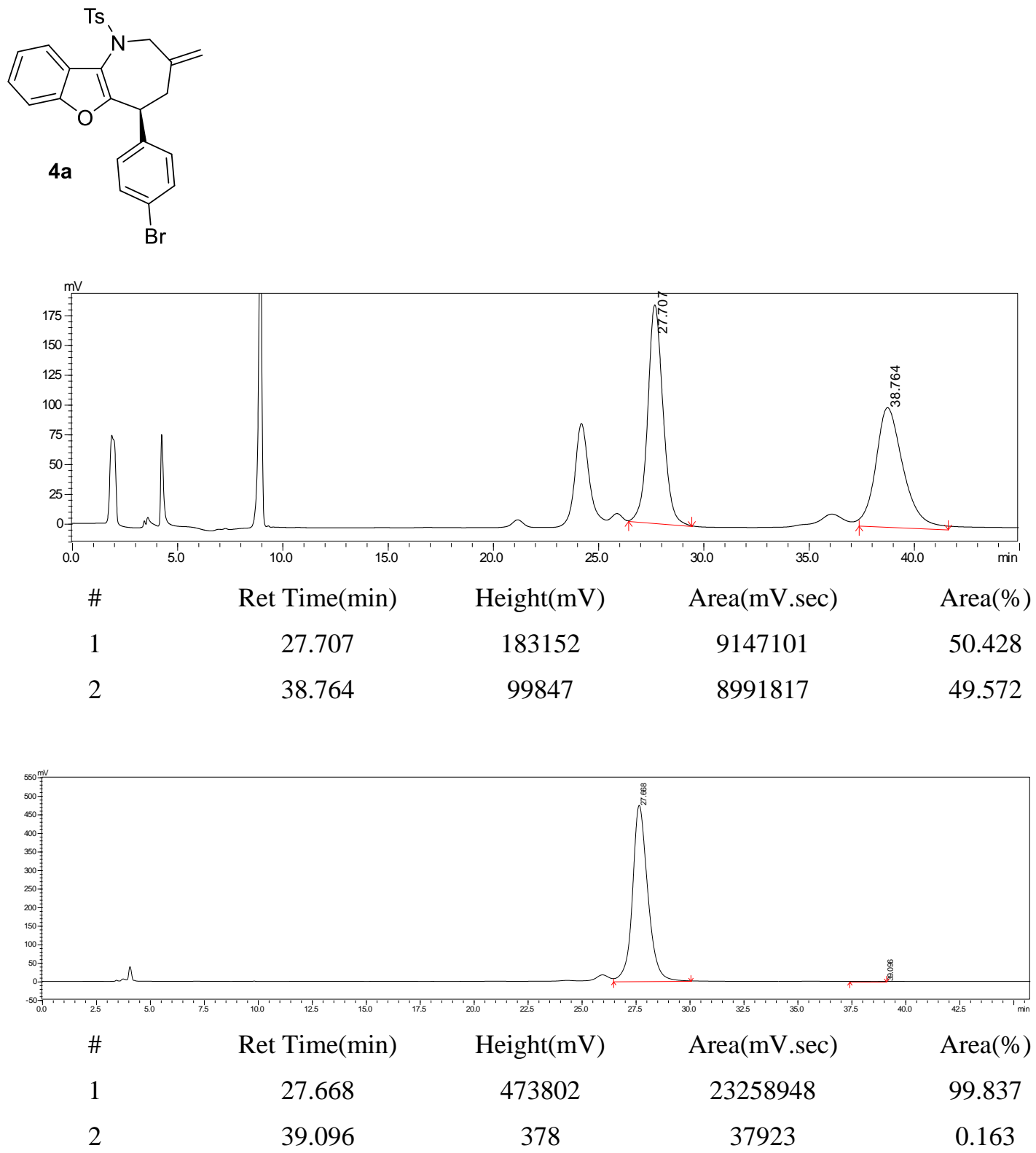

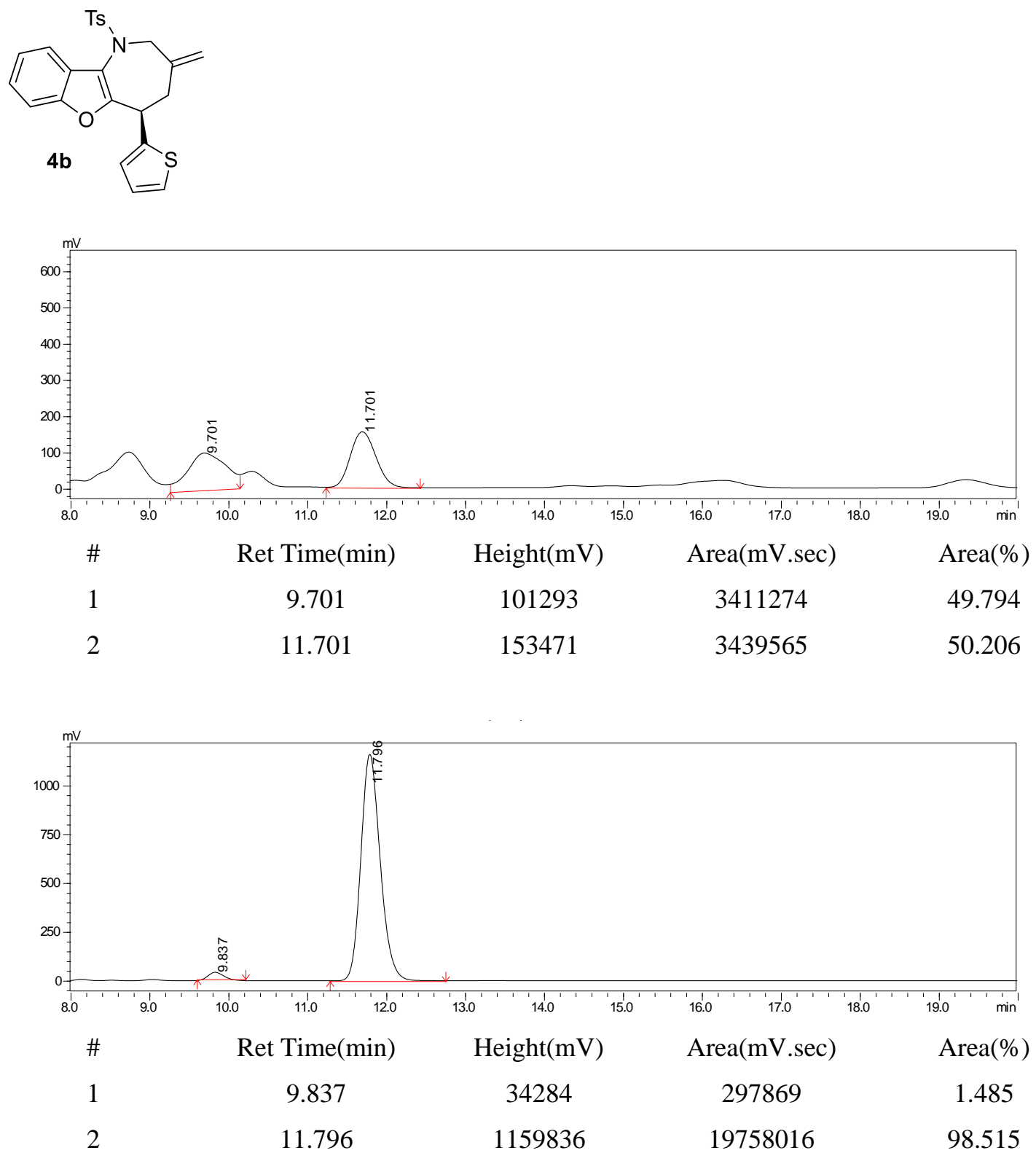

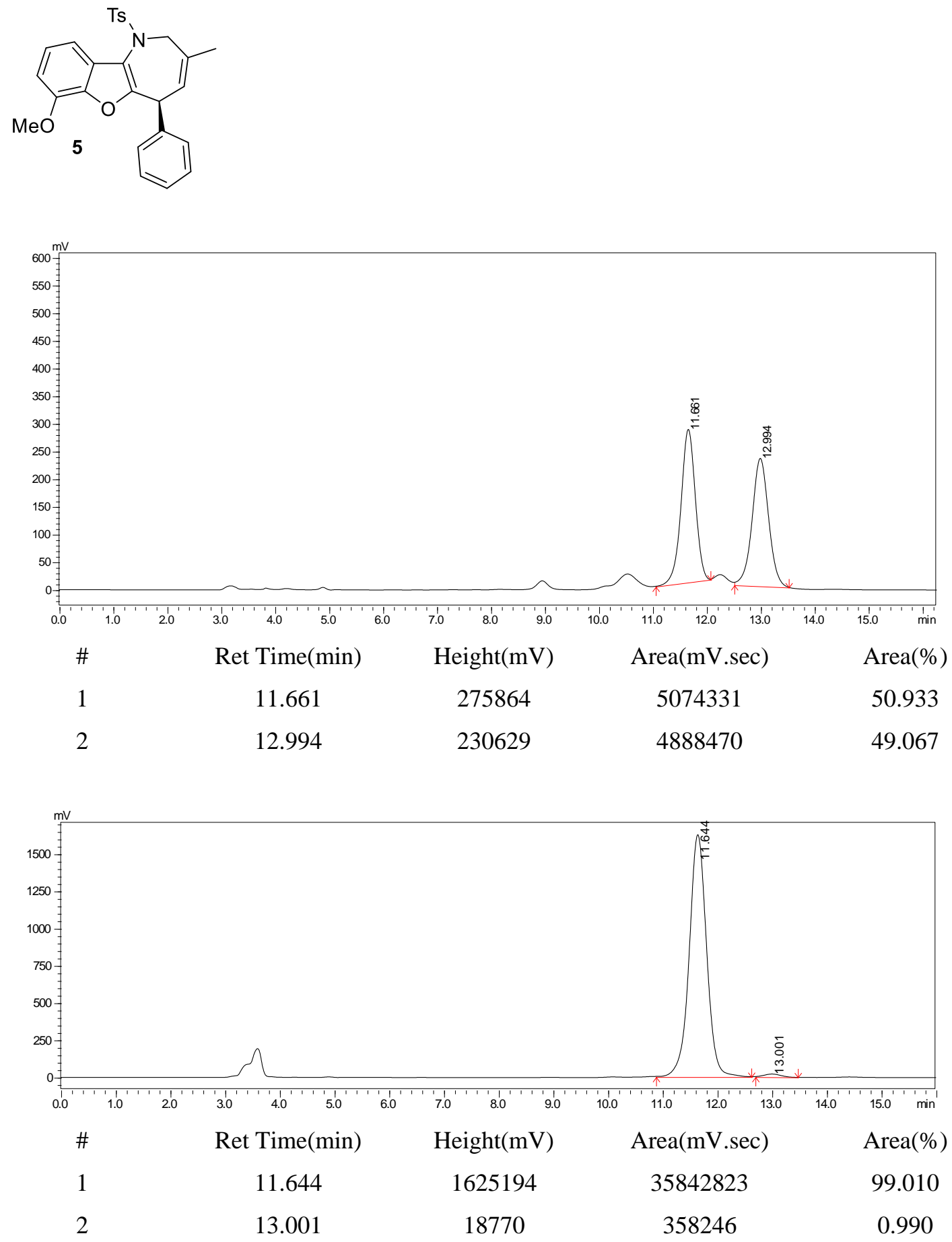

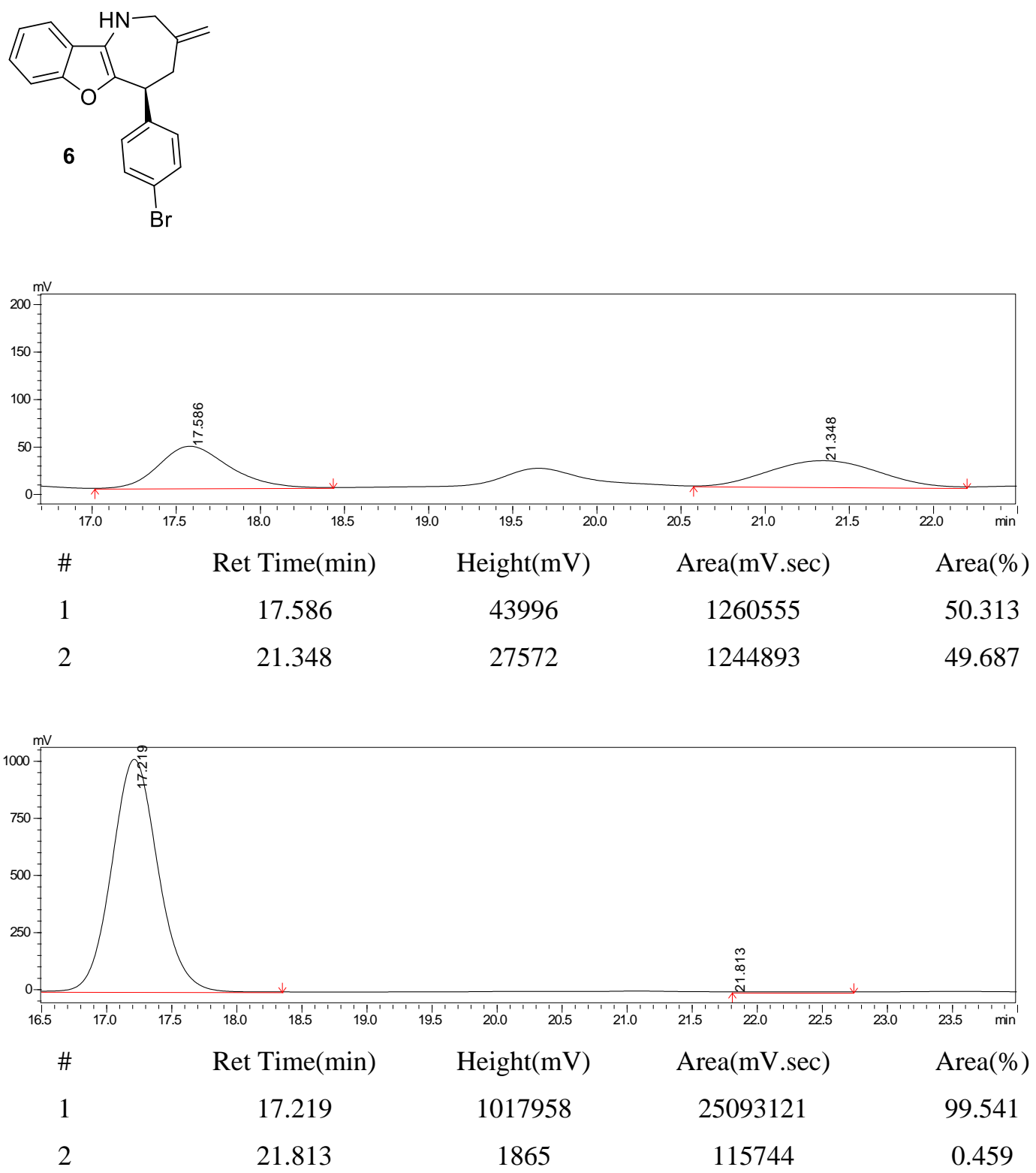